\title{
Tame Supercuspidal Representations of GL(n) Distinguished by a Unitary Group
}

\author{
To the Memory of Hanna Miriam Sandler \\ JEFFREY HAKIM ${ }^{1, \star}$ and FIONA MURNAGHAN ${ }^{2, \star \star}$ \\ ${ }^{1}$ Department of Mathematics and Statistics, American University, Washington, \\ DC 20016-8050,U.S.A.e-mail: jhakim@american.edu \\ ${ }^{2}$ Department of Mathematics, University of Toronto, Toronto, Canada, M5S $3 G 3$
}

(Received: 11 August 2000; accepted: 24 August 2001)

\begin{abstract}
This paper analyzes the space $\operatorname{Hom}_{H}(\pi, 1)$, where $\pi$ is an irreducible, tame supercuspidal representation of GL( $n)$ over a $p$-adic field and $H$ is a unitary group in $n$ variables contained in $\operatorname{GL}(n)$. It is shown that this space of linear forms has dimension at most one. The representations $\pi$ which admit nonzero $H$-invariant linear forms are characterized in several ways, for example, as the irreducible, tame supercuspidal representations which are quadratic base change lifts.
\end{abstract}

Mathematics Subject Classifications (2000). 22E50, 22E35, 11F70.

Key words. supercupsoidal representations, distinguished representations, quadratic base change.

\section{Introduction}

The theory of quadratic base change for automorphic representations of $\operatorname{GL}(n)$ has been studied extensively (for example, $[2,4,17,22,28,30]$ ) and has had numerous applications to number theory, including Wiles' celebrated proof of Fermat's Last Theorem. The theory relies on establishing the existence of local base change maps and, at present, this is achieved via global techniques involving adelic trace formulas. As efficient as the global techniques are, they tell us relatively little about the fine structure of the local representations in the image of the local base change maps. The point of the present paper is to provide a detailed study of the tame supercuspidal representations which arise in this manner.

The representations in question are traditionally characterized in terms of a character relation or an invariance property under a Galois action. The global theory suggests that they may also be described in terms of harmonic analysis on the analogues of symmetric spaces over non-Archimedean local fields. In particular, if $F / F^{\prime}$ is a quadratic extension of non-Archimedean local fields of characteristic zero and odd residual characteristic, we show in Theorem 4.4 that a tame supercuspidal

\footnotetext{
${ }^{\star}$ Research supported in part by NSA grant \#MDA904-99-1-0065.

${ }^{\star \star}$ Research supported in part by NSERC.
} 
representation $\left(\pi, V_{\pi}\right)$ of $G=\mathrm{GL}(n, F)$ is a base change lift from $\mathrm{GL}\left(n, F^{\prime}\right)$ precisely when it is distinguished by a unitary group in $n$ variables $H \subset G$ in the sense that $\operatorname{Hom}_{H}(\pi, 1) \neq 0$. The latter space consists of those linear forms $\Lambda$ on $V_{\pi}$ such that $\Lambda(\pi(h) v)=\Lambda(v)$, for all $h \in H$ and $v \in V_{\pi}$. The result described above was conjectured by Jacquet and Ye $[19,20]$ for quasisplit unitary groups. In the case in which $F / F^{\prime}$ is unramified and the unitary group is quasisplit, a proof has also just been obtained by D. Prasad [27], with no assumption of tameness, using entirely different methods than ours.

The original motivation for our paper lies in a more difficult conjecture of Jacquet, which asserts that if $\pi$ is any irreducible, supercuspidal representation of $G=\mathrm{GL}(n, F)$ and $H$ is a unitary subgroup in $n$ variables then the dimension of $\operatorname{Hom}_{H}(\pi, 1)$ is at most one. In Theorem 3.3, we prove that this conjecture holds for tame supercuspidal representations. In particular, if the residue field of $F$ has characteristic $p>n$ then the conjecture holds for all irreducible, supercuspidal representations. The importance of such 'multiplicity one' results in harmonic analysis over both local and global fields is made evident, for example, by the prominent role played by Whittaker models in the theory of automorphic representations. In our setting, the multiplicity one property says that the representation space of an $H$-distinguished representation $\pi$ has a canonical model as a space of functions on $H \backslash G$. Indeed, Frobenius reciprocity correlates the existence of a nonzero linear form in $\operatorname{Hom}_{H}(\pi, 1)$ with a model for the representation space of $\pi$ as a space of functions on $H \backslash G$ and, therefore, the $H$-distinguished representations of $G$ may be viewed those representations which contribute to the harmonic analysis on $H \backslash G$.

Another major objective of this paper is to describe the $H$-distinguished tame supercuspidal representations $\pi$ of $G$ in terms of the inducing data in Howe's construction. Suppose, for example, that $\pi$ is associated to an admissible quasicharacter $\theta$ of $E^{\times}$, where $E / F$ is a tamely ramified extension of degree $n$. We show that there must exist an automorphism $\sigma$ of $E$ which restricts to the nontrivial element of $\operatorname{Gal}\left(F / F^{\prime}\right)$ and is such that $\theta=\theta^{\prime} \circ N_{E / E^{\prime}}$, for some quasicharacter $\theta^{\prime}$ of the multiplicative group of the fixed field $E^{\prime}$ of $\sigma$. Moreover, we prove in Proposition 6.1 that $\theta^{\prime}$ must be admissible over $F^{\prime}$ and the quasicharacters which arise in a Howe factorization of $\theta^{\prime}$ lift by quadratic base change to give a Howe factorization of $\theta$.

Let us now briefly outline the contents of the paper. According to Howe's construction, a tame supercuspidal representation $\pi$ is realized as an induced representation from a certain subgroup $R$ of $G$ which depends on the admissible quasi-character $\theta$ which parametrizes $\pi$. The subgroup $R$ is a product of various factors. The main point of Section 2 is to show that these factors may be chosen in a way which is compatible with the involution which defines the unitary group $H$. In Section 3, we state the multiplicity one result discussed above, Theorem 3.3, for $H$-distinguished tame supercuspidal representations and we discuss corresponding properties involving unitary similitude groups. Various equivalent characterizations of $H$-distinguished tame supercuspidal representations are given in Theorem 
4.4. Finite field analogues are also discussed in Section 4. A global argument is described in Section 5 which proves that if $\pi$ is $H$-distinguished then it must be Galois invariant or, equivalently, a base change lift from $\operatorname{GL}\left(n, F^{\prime}\right)$. The details of this argument appear in a separate paper [10]. The basic notions associated with admissible quasicharacters and their Howe factorizations are recalled in Section 6. As mentioned above, we show how quadratic base change lifting preserves Howe factorizations. In Section 7, we present the facts needed for Howe's construction which involve Heisenberg and Weil representations over the field $\mathbb{F}_{p}$, where $p$ is the characteristic of the various residue fields. Lemma 7.1 gives a simple description of when a Heisenberg group over $\mathbb{F}_{p}$ is distinguished with respect to an arbitrary subgroup. Proposition 7.2 says that if the subgroup is a maximal isotropic subspace then the Heisenberg representation is always distinguished and the space of invariant linear forms has dimension one.

In Section 8, we review Howe's construction of tame supercuspidal representations as induced representations $\pi=\operatorname{Ind}_{R}^{G}(\kappa)$ from compact-mod-center subgroups $R$. Here, we use the content of Sections 2, 6 and 7. A key technical device which is used in our constructions of Heisenberg and Weil representations is that the \pm 1 eigenspaces of the involution defining the Lie algebra of $H$ give rise to canonical polarizations of the relevant symplectic spaces. This choice of polarization, which is discussed in Section 8 , does not necessarily agree with the standard canonical polarizations which occur in the literature of tame supercuspidal representations. Therefore, it is necessary to discuss the effect of varying the polarization and this is done in Section 9.

After having laid out some of the groundwork for the proofs of Theorems 3.3 and 4.4 in Sections 2-9, the heart of the proofs is developed in Sections 10-14. In Section 11 , the basic problems are reduced to problems regarding compact groups. Lemma 11.2 involves an application of Mackey Theory to decompose $\operatorname{Hom}_{H}(\pi, 1)$ into pieces of the form $\operatorname{Hom}_{R \cap g \mathrm{Hg}^{-1}}(\kappa, 1)$, as $g$ ranges over the double coset space $R \backslash G / H$. Proposition 12.1 states that all of these pieces vanish, except for $\operatorname{Hom}_{R \cap H}(\kappa, 1)$. For a given $\pi$, the inducing representation $\kappa$ is a tensor product $\kappa_{1} \otimes \cdots \otimes \kappa_{r}$ of representations $\kappa_{i}$ attached to the factors in the Howe factorization of the admissible quasicharacter which parametrizes $\pi$. We show in Proposition 13.1 that the elements of the spaces $\operatorname{Hom}_{R \cap H}(\kappa, 1)$ have factorizations, where the factors lie in certain spaces $\operatorname{Hom}_{R \cap H}\left(\kappa_{i}, \vartheta_{i}\right)$. The latter spaces are computed mainly in Section 10, with one exceptional case deferred until Section 12. (See Proposition 10.1 and Proposition 12.3.) In the final section of the paper, we apply some techniques due to Jeff Adler to prove the existence of distinguished tame supercuspidal representations.

The work in this paper follows up initial investigations carried out by the first author together with Zhengyu Mao [8, 9]. There, preliminary examples of $H$-distinguished representations are obtained from distinguished representations associated to various subgroups of GL( $n)$ over a finite field. Other types of distinguished tame supercuspidal representations of GL( $n)$ arise in applications involving the reducibility of certain induced representations studied in papers by the second author and Joe Repka $[25,26]$. Conditions on the inducing data of the distinguished 
representations which appear in the latter two papers are very similar to conditions obtained in the present paper. The methods developed in this paper to detect distinguishedness of tame supercuspidal representations by unitary groups of rank $n$ can be applied more generally. In particular, if $H$ is replaced by $\operatorname{GL}\left(n, F^{\prime}\right)$ or by $\operatorname{GL}(n / 2, F) \times \operatorname{GL}(n / 2, F)$, if $n$ is even, these methods have been used to determine which tame supercuspidal representations are $H$-distinguished [12]. Preliminary investigations [11] indicate that a similar approach applies when $H$ is an orthogonal group of rank $n$.

\section{Embeddings}

Let $F$ be a finite extension of $\mathbb{Q}_{p}$, for some odd prime $p$, and assume $E$ is a tamely ramified extension of $F$ of degree $n$. In Section 8, we will recall Howe's method of associating to each admissible quasicharacter of $E^{\times}$an irreducible, supercuspidal representation of $G=\mathrm{GL}(n, F)$, customizing the construction where necessary to make it compatible with the involution defining our unitary group. It is these representations which we refer to as 'tame supercuspidal representations' throughout the paper.

The first step in Howe's construction is to fix embeddings of the relevant fields in the Lie algebra $\mathfrak{g}=\mathfrak{g l}(n, F)$. Assume $\sigma$ and $\tau$ are automorphisms of order two of $E$ and $F$, respectively, such that $\sigma$ restricts to $\tau$. The fixed fields of the automorphisms $\sigma$ and $\tau$ are denoted by $E^{\prime}$ and $F^{\prime}$, respectively. In general, if $L$ is a $p$-adic field, we use the notation $\mathfrak{D}_{L}$ for the ring of integers of $L$ and we let $\mathfrak{B}_{L}$ denote the maximal ideal of $\mathfrak{S}_{L}$. Let $G=\operatorname{GL}(n, F)$ and $\mathfrak{g}=\mathfrak{g l}(n, F)$. If $x \in \mathfrak{g}$ then $x^{*}$ denotes the matrix given by applying $\tau$ to the entries of ${ }^{t} x$. If $x^{*}=x$ we say that $x$ is Hermitian. The group $G$ acts on the set of Hermitian matrices in $G$ by $g \cdot x=g x g^{*}$. According to a classical result of Jacobson, this action has two orbits and the orbit of a Hermitian matrix $x \in G$ is determined by its discriminant, that is, the class of $\operatorname{det} x$ in $F^{\prime \times} / N_{F / F^{\prime}}\left(F^{\times}\right)$. (A very different classification holds over Archimedean fields in terms of the signature of a Hermitian matrix.) When two Hermitian matrices lie in the same $G$-orbit, we say that they are similar.

PROPOSITION 2.1. There exists an embedding of $E$ in $\mathfrak{g}$ such that if $E$ is identified with its image in $\mathfrak{g}$ then $\sigma(x)=x^{*}$, for all $x \in E$. Consequently, the elements of $E^{\prime}$ correspond to Hermitian matrices.

The proof of Proposition 2.1 will require the following lemma:

LEMMA 2.2. $N_{E^{\prime} / F^{\prime}}\left(E^{\prime \times}\right) \nsubseteq N_{F / F^{\prime}}\left(F^{\times}\right)$.

Proof. A similar result appears in [26]. It is useful to recall the proof here, since it makes evident several distinct cases which lie beneath the surface throughout this paper. Following [26], to obtain an element $\alpha \in E^{\prime \times}$ such that $N_{E / F}(\alpha)=N_{E^{\prime} / F^{\prime}}(\alpha) \notin$ $N_{F / F^{\prime}}\left(F^{\times}\right)$, we can take $\alpha$ to be any nonsquare root of unity in $E^{\prime}$ if $E / E^{\prime}$ is ramified, and we can take $\alpha$ to be any prime element in $E^{\prime}$ otherwise. 
Case 1: $E / E^{\prime}$ and $F / F^{\prime}$ both unramified. In this case, by uniqueness of unramified extensions, the residual degree $f(E / F)$ must be odd. If $\varpi_{E^{\prime}}$ is a prime element for $E^{\prime}$ then $N_{E / F}\left(\varpi_{E^{\prime}}\right)$ has odd valuation, and therefore cannot be a norm from $F$, as $F / F^{\prime}$ is unramified.

Case 2: $E / E^{\prime}$ ramified. In this case, by Lemma 5.4 of [25], $F / F^{\prime}$ is ramified and the ramification degrees $e(E / F)=e\left(E^{\prime} / F^{\prime}\right)$ are odd. Let $E_{\text {un }}^{\prime}$ be the maximal unramified extension of $F^{\prime}$ contained in $E^{\prime}$. Then, as $E^{\prime} / E_{\text {un }}^{\prime}$ is totally ramified, there is no loss of generality in taking $\alpha$ to be a nonsquare root of unity in $E_{\text {un }}^{\prime}$. Hence, $N_{E^{\prime} / E_{\text {un }}^{\prime}}(\alpha)=\alpha^{e\left(E^{\prime} / F^{\prime}\right)}$ is a nonsquare, since $e\left(E^{\prime} / F^{\prime}\right)$ is odd. Now if $N_{E_{\text {un }}^{\prime} / F^{\prime}}\left(\alpha^{e\left(E^{\prime} / F^{\prime}\right)}\right)$ were a square in $\mathfrak{O}_{F^{\prime}}^{\times}$, it would force $N_{E_{\text {un }}^{\prime} / F^{\prime}}\left(\mathfrak{D}_{E_{\text {un }}^{\prime}}^{\times}\right)$to consist of squares, which, by the surjectivity of the norm map over finite fields, is not the case. Thus $N_{E^{\prime} / F^{\prime}}(\alpha)$ is a nonsquare in $\mathfrak{D}_{F^{\prime}}^{\times}$, and, as $F / F^{\prime}$ is ramified, a nonsquare in $\mathfrak{D}_{F^{\prime}}^{\times}$cannot be a norm from $F$.

Case 3: $E / E^{\prime}$ unramified and $F / F^{\prime}$ ramified. Let $\varepsilon$ be a nonsquare root of unity in $E_{\text {un }}^{\prime}$. Pick a prime element $\varpi \in F^{\prime}$ such that $F=F^{\prime}(\sqrt{\varpi})$. The compositum $F E_{\text {un }}^{\prime}$ is just $E_{\text {un }}^{\prime}(\sqrt{\varpi})$. As $E^{\prime} / E_{\text {un }}^{\prime}$ is totally ramified, $\varepsilon$ is a nonsquare in $E^{\prime}$ and hence $E=E^{\prime}(\sqrt{\varepsilon})$ and $\sigma(\varepsilon)=-\sqrt{\varepsilon}$. So $\sqrt{\varepsilon \varpi} \in E^{\prime}$ and $E_{\text {un }}^{\prime}(\sqrt{\varepsilon \varpi})$ is a quadratic ramified extension of $E_{\mathrm{un}}^{\prime}$. Now $N_{E^{\prime} / E_{\mathrm{un}}^{\prime}}\left(\varpi_{E^{\prime}}\right)$ is a prime element in $E_{\mathrm{un}}^{\prime}$, and by transitivity of norm, is a norm from $E_{\text {un }}^{\prime}(\sqrt{\varepsilon \varpi})$. That is, $N_{E^{\prime} / E_{\text {un }}^{\prime}}\left(\varpi_{E^{\prime}}\right) \in-b^{2} \varepsilon \varpi+\mathfrak{B}_{E_{\text {un }}^{\prime}}^{2}$, for some $b \in \mathfrak{S}_{E_{\text {un }}^{\prime}}^{\times}$. Note that $-\varpi=N_{F E_{\text {un }}^{\prime} / E_{\text {un }}^{\prime}}(\sqrt{\varpi})$. Hence, we have $N_{E^{\prime} / E_{\text {un }}^{\prime}}\left(\varpi_{E^{\prime}}\right) \in$ $\varepsilon N_{F E_{\text {un }}^{\prime} / E_{\text {un }}^{\prime}}\left(F E_{\text {un }}^{\prime \times}\right)$. This implies

$$
N_{E^{\prime} / F^{\prime}}\left(\varpi_{E^{\prime}}\right) \in N_{E_{\mathrm{un}}^{\prime} / F^{\prime}}(\varepsilon) N_{F E_{\mathrm{un}}^{\prime} / F^{\prime}}\left(F E_{\mathrm{un}}^{\prime \times}\right) \subset N_{E_{\mathrm{un}}^{\prime} / F^{\prime}}(\varepsilon) N_{F / F^{\prime}}\left(F^{\times}\right) .
$$

It follows that $N_{E^{\prime} / F^{\prime}}\left(\varpi_{E^{\prime}}\right) \in N_{F / F^{\prime}}\left(F^{\times}\right)$if and only if $N_{E_{\text {un }}^{\prime} / F^{\prime}}(\varepsilon) \in N_{F / F^{\prime}}\left(F^{\times}\right)$. Recalling that both $F E_{\text {un }}^{\prime} / E_{\text {un }}^{\prime}$ and $F / F^{\prime}$ are ramified and $\varepsilon$ is a nonsquare root of unity in $E_{\text {un }}^{\prime}$, we see that if we replace $E^{\prime}$ by $E_{\text {un }}^{\prime}$ and $E$ by $F E_{\text {un }}^{\prime}$, we are in the situation of Case 2 and, hence, $N_{E_{\text {un }}^{\prime} / F^{\prime}}(\varepsilon) \notin N_{F / F^{\prime}}\left(F^{\times}\right)$.

Proof of Proposition 2.1. Fix an $F^{\prime}$-basis $\left\{\xi_{1}, \ldots, \xi_{n}\right\}$ of $E^{\prime}$. View the elements of $F^{n}$ as column vectors and define an $F$-linear isomorphism $v: E \cong F^{n}$ by $v\left(\sum_{i} x_{i} \xi_{i}\right)=$ ${ }^{t}\left(x_{1}, \ldots, x_{n}\right)$. When $x \in E$, we let $i(x) \in \mathfrak{g}$ be the matrix which satisfies $i(x) v(y)=v(x y)$, for all $y \in E$. In other words, $i: E \hookrightarrow \mathfrak{g}$ is just the regular representation of $E$ associated to the given basis. Define a nondegenerate Hermitian form (with respect to $F / F^{\prime}$ ) on $E$ by $\langle x, y\rangle=\operatorname{tr}_{E / F}(x \sigma(y)$ ). The matrix of this Hermitian form with respect to $v$ is the symmetric matrix $\xi$ whose $i j$ th entry is $\xi_{i j}=\operatorname{tr}_{E^{\prime} / F^{\prime}}\left(\xi_{i} \xi_{j}\right)$. If $x, y, z \in E$ then $\langle\sigma(x) y, z\rangle=\langle y, x z\rangle$ and this implies that $i(\sigma(x))^{*} \xi=\xi i(x)$.

Suppose that $\xi=g^{*} g$, for some $g \in G$. The embedding of $E$ in $g$ given by identifying $x \in E$ with $g i(x) g^{-1}$ satisfies the desired relation $\sigma(x)=x^{*}$, for all $x \in E$. It remains to consider the case in which $\xi=g^{*} g$ cannot be solved or, equivalently, $\xi$ is not similar to the identity matrix. Assuming we are in this case, then, according to Lemma 2.2, we may choose $\alpha \in E^{\prime \times}$ such that $N_{E^{\prime} / F^{\prime}}(\alpha) \notin N_{F / F^{\prime}}\left(F^{\times}\right)$. 
Note that $\xi i(\alpha)$ is Hermitian. The determinant of $i(\alpha)$ does not lie in $N_{F / F^{\prime}}\left(F^{\times}\right)$, since $\operatorname{det} i(\alpha)=N_{E / F}(\alpha)=N_{E^{\prime} / F^{\prime}}(\alpha)$. Therefore, there must exist $g \in G$ such that $\xi i(\alpha)=g^{*} g$. Again, $x \mapsto g i(x) g^{-1}$ is an embedding of $E$ in $g$ with the desired property.

In [15], Howe associates to each quasicharacter $\theta$ of $E$ which is admissible over $F$ a tower of intermediate fields of $E / F$ :

$$
F=E_{0} \subsetneq E_{1} \subsetneq \cdots \subsetneq E_{r}=E .
$$

The relation between $\theta$ and the associated tower will be recalled later, but this is irrelevant for our immediate purposes. For the quasicharacters we consider, it will turn out that all of the subfields $E_{i}$ are stable under $\sigma$ and if $E_{i}^{\prime}$ is the fixed field of $\sigma \mid E_{i}$ then $E_{i}$ is a quadratic extension of $E^{\prime}{ }_{i}$ and, in addition, $E_{i}=E^{\prime}{ }_{i} F$. Thus, the fields $E_{i}^{\prime}$ form a tower

$$
F^{\prime}=E_{0}^{\prime} \subsetneq E^{\prime}{ }_{1} \subsetneq \cdots \subsetneq E_{r}^{\prime}=E^{\prime} .
$$

For each index $i$, let

$$
n_{i}=\left[E: E_{i}\right]=\left[E^{\prime}: E_{i}^{\prime}\right] \text { and } n_{i}^{\prime}=\left[E_{i}: F\right]=\left[E_{i}^{\prime}: F^{\prime}\right] \text {. }
$$

Let $\mathfrak{g}_{i}$ denote the Lie algebra $\mathfrak{g l}\left(n_{i}, E_{i}\right)$.

We will now construct an embedding of $E$ in $g$ that has the property that $\sigma(x)=x^{*}$ for all $x \in E$. Let us stress at the outset, however, that we will not be applying Proposition 2.1 directly to the extension $E / F$. Rather, we apply it the extensions $E_{i} / E_{i-1}$ and move up the tower of subfields of $E$ one step at a time.

The first step is to apply Proposition 2.1 to $E_{1} / F$ to obtain an embedding of $\mathfrak{g}_{1}$ in $\mathfrak{g}$, using the fact that $\mathfrak{g}_{1}=\mathfrak{g l}\left(\left[E: E_{1}\right], E_{1}\right)$ and $\mathfrak{g}=\mathfrak{g l}\left(\left[E: E_{1}\right], \mathfrak{g l}\left(\left[E_{1}: F\right], F\right)\right)$. Moving up the tower of subfields, we similarly obtain embeddings $\mathfrak{g}_{i} \hookrightarrow \mathfrak{g}_{i-1}$ which we regard as inclusions. Thus, we have

$$
E=\mathfrak{g}_{r} \subsetneq \mathfrak{g}_{r-1} \subsetneq \cdots \subsetneq \mathfrak{g}_{0}=\mathfrak{g} .
$$

Note that $\mathfrak{g}_{i}$ is a space of $n_{i} \times n_{i}$ block matrices in $\mathfrak{g}_{i-1}$, where the blocks of $x \in \mathfrak{g}_{i}$ lie in $E_{i} \subset \mathfrak{g l}\left(\left[E_{i}: E_{i-1}\right], E_{i-1}\right)$. We also observe that $\mathfrak{g}_{i}$ is the centralizer of $E_{i}$ in $\mathfrak{g}$. Similarly, there is a chain of groups

$$
E^{\times}=G_{r} \subsetneq G_{r-1} \subsetneq \cdots \subsetneq G_{0}=G,
$$

where $G_{i}=\mathrm{GL}\left(n_{i}, E_{i}\right)$ is the centralizer of $E_{i}$ in $G$.

Suppose $e_{1}, \ldots, e_{n}$ is an $F$-basis of $E$. This gives an identification of $E$ with $F^{n}$, viewed as a space of column vectors. If $x \in E$ then multiplication by $x$ determines an $F$-linear transformation of $E$ and hence a matrix in $g$. Thus we have an embedding of $E$ in $g$ associated to the given basis and we refer to this as the regular representation of $E$ in $g$ associated to $e_{1}, \ldots, e_{n}$. It is easy to verify that given any embedding of $E$ in $g$ there exists a basis $e_{1}, \ldots, e_{n}$ such that the associated regular representation is identical to the given embedding. (In fact, the basis is unique if we assume, in addition, that $e_{1}=1$.) 
Let us assume that we have chosen $e_{1}, \ldots, e_{n}$ so that the regular representation is just the embedding of $E$ we have fixed above. Using the associated identification of $E$ with $F^{n}$, we identify the fractional ideals $\mathfrak{P}_{E}^{k}$ in $E$ with lattices in $F^{n}$. This lattice flag in $F^{n}$ gives rise to the filtration of $\mathfrak{g}$ by the parahoric $\mathfrak{S}_{F}$-algebras

$$
\mathfrak{b}_{j}=\left\{x \in \mathfrak{g}: x \mathfrak{P}_{E}^{k} \subset \mathfrak{P}_{E}^{j+k}, \quad \text { for all integers } k\right\} .
$$

Let $\mathfrak{b}=\mathfrak{b}_{0}$. The corresponding parahoric subgroups are defined by $B=B_{0}=\mathfrak{b}^{\times}$and $B_{j}=1+\mathfrak{b}_{j}$, for $j>0$. When $0 \leqslant i \leqslant r$, we take $\mathfrak{b}_{j, i}=\mathfrak{b}_{j} \cap \mathfrak{g}_{i}$ and when, additionally, $j$ is nonnegative, we let $B_{j, i}=B_{j} \cap G_{i}$.

The next result is a refinement of Proposition 2.1 in the case in which $E^{\prime} / F^{\prime}$ is unramified.

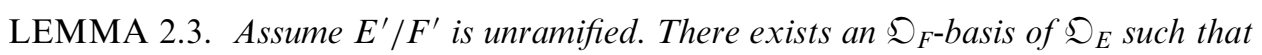
if $E$ is embedded in $\mathrm{g}$ via the regular representation associated to this basis then $\sigma(x)=x^{*}$, for all $x \in E$.

Proof. Our proof is obtained by refining the proof of Proposition 2.1 as follows. Assume the basis $\xi_{1}, \ldots, \xi_{n}$ in the proof of Proposition 2.1 is an $\mathfrak{D}_{F^{\prime}}$-basis of $\mathfrak{D}_{E^{\prime}}$. Let $D$ be the discriminant ideal in $\mathfrak{D}_{F^{\prime}}$ associated to $\mathfrak{S}_{E^{\prime}}$, that is, the ideal generated by the determinant of $\xi$. Let $\mathfrak{D}$ be the different of $\mathfrak{D}_{E^{\prime}}$ with respect to $\mathfrak{D}_{F^{\prime}}$ or, in other words, the ideal of $\mathfrak{S}_{E^{\prime}}$ whose inverse as a fractional ideal is the largest fractional ideal $I$ such that $\operatorname{tr}_{E^{\prime} / F^{\prime}}(I) \subset \mathfrak{S}_{F^{\prime}}$. The discriminant and the different are related by $\mathfrak{D}=N_{E^{\prime} / F^{\prime}}(\mathfrak{D})$ and, since $E^{\prime} / F^{\prime}$ is unramified, we have $\mathfrak{D}=\mathfrak{D}_{E^{\prime}}$. (See Proposition $6, \S 3$ and Corollary 1 of Theorem $1, \S 5$ in Chapter III of [29].) It follows that the symmetric matrix $\xi$ in the proof of Proposition 2.1 lies in $\operatorname{GL}\left(n, \mathfrak{D}_{F^{\prime}}\right)$.

Let $K=\operatorname{GL}\left(n, \mathfrak{D}_{F}\right)$. The $K$-orbits of Hermitian matrices in $G$, where $K$ acts by $k \cdot x=k x k^{*}$, were originally described by Jacobowitz in [16]. (See also [9].) One can restrict Jacobowitz' result to obtain a description of the $K$-orbits of hermitian matrices in $K$. When $F / F^{\prime}$ is unramified, then there is a single orbit. When $F / F^{\prime}$ is ramified, there are two orbits, one is represented by the identity matrix and the other is represented by any diagonal matrix with diagonal $(1, \ldots, 1, \delta)$, where $\delta \in \mathfrak{D}_{F^{\prime}}^{\times}$is a nonsquare in the residue field of $F^{\prime}$.

Assume first that $\xi=k^{*} k$, for some $k \in K$. Then $x \mapsto k i(x) k^{-1}$ is an embedding of $E$ in $\mathrm{g}$ of the desired type. Now suppose $\xi=k^{*} k$ has no solutions $k \in K$. Then $F / F^{\prime}$ must be ramified. Since $E^{\prime} / F^{\prime}$ is unramified, $E / F$ must also be unramified. It follows that $E / E^{\prime}$ must be ramified. We therefore are in case (ii) in the proof of Lemma 2.2. In this case, the element $\alpha$ is chosen to be a root of unity. The matrix $\xi i(\alpha)$ is hermitian and lies in $K$, but it cannot lie in the $K$-orbit of $\xi$ since it does not lie in the $G$-orbit of $\xi$. Therefore, $\xi i(\alpha)=k^{*} k$ must have a solution $k \in K$. For such a solution $k$, a suitable embedding is again given by $x \mapsto k i(x) k^{-1}$.

The following invariance properties will be used implicitly throughout the paper:

LEMMA 2.4. If $i$ and $j$ are integers and $0 \leqslant i \leqslant r$ then $\mathfrak{g}_{i}^{*}=\mathfrak{g}_{i}$ and $\mathfrak{b}_{j}^{*}=\mathfrak{b}_{j}$. If $\eta \in E^{\prime \times}$ and $\sigma_{\eta}$ is defined on $\mathfrak{g}$ by $\sigma_{\eta}(x)=\eta x^{*} \eta^{-1}$ then $\sigma_{\eta}\left(\mathfrak{g}_{i}\right)=\mathfrak{g}_{i}$ and $\sigma_{\eta}\left(\mathfrak{b}_{j}\right)=\mathfrak{b}_{j}$. 
Proof. It is well known that $\mathfrak{g}_{i}$ is the centralizer of $E_{i}$ in $\mathfrak{g}$ and $\mathfrak{b}$ is the intersection of all maximal orders in $\mathfrak{g}$ which contain $\mathfrak{D}_{E}$. All of the assertions follows readily from these characterizations of $\mathfrak{g}_{i}$ and $\mathfrak{b}$.

\section{Unitary Groups and Similitude Groups}

Fix a Hermitian matrix $\eta \in G$. Associated to $\eta$ is a unitary group $H_{\eta}=\{g \in G$ : $\left.g \eta g^{*}=\eta\right\}$, a unitary similitude group $H_{\eta}^{\prime}=\left\{g \in G: g \eta g^{*} \eta^{-1} \in F^{\times}\right\}$, and a homomorphism $\mu_{\eta}: H_{\eta}^{\prime} \rightarrow F^{\times}$defined by $g \eta g^{*}=\mu_{\eta}(g) \eta$, for $g \in H_{\eta}^{\prime}$. (Note that it is common in the literature to define 'the unitary group associated to $\eta$ ' by the condition $g^{*} \eta g=\eta$, rather than $g \eta g^{*}=\eta$. This convention produces the group $H_{\eta^{-1}}=\eta^{-1} H_{\eta} \eta$.)

In this paper, we are mainly interested in unitary groups, but we will make some elementary remarks which might be helpful in applying our results for unitary groups to similitude groups. Before doing this, let us state some simple facts about unitary groups. We observe, first of all, that if $g \in G$ then $g H_{\eta} g^{-1}=H_{g \eta g^{*}}$. Consequently, the conjugacy class of the unitary group $H_{\eta}$ is determined by the $G$-orbit of the Hermitian matrix $\eta$, relative to the action $g \cdot \eta=g \eta g^{*}$. As mentioned in the previous section, there are two $G$-orbits of Hermitian matrices in $G$. However, when $n$ is odd it turns out that the two associated conjugacy classes of unitary groups are identical. Indeed, if $n$ is odd then we may take $\eta_{1}=1$ and $\eta_{2} \in F^{\prime \times}-N_{F / F^{\prime}}\left(F^{\times}\right)$as representatives of the two orbits of hermitian matrices. But then the associated unitary groups $H_{\eta_{1}}$ and $H_{\eta_{2}}$ are identical, since $\eta_{1}$ and $\eta_{2}$ both lie in the center of $G$. On the other hand, when $n$ is even the two conjugacy classes of unitary groups will be distinct.

In general, if $n$ is even or odd, if one takes $\eta$ to be an antidiagonal Hermitian matrix then $H_{\eta}$ is a quasisplit unitary group with a Borel subgroup consisting of upper triangular matrices. If $n$ is odd then all unitary groups must therefore be quasisplit. If $n$ is even then $H_{\eta}$ is quasisplit exactly when $(-1)^{n / 2} \operatorname{det} \eta \in$ $N_{F / F^{\prime}}\left(F^{\times}\right)$.

Our interest is in studying the irreducible tame supercuspidal representations $\left(\pi, V_{\pi}\right)$ of $G$ which are $H_{\eta}$-distinguished in the sense that the space $\operatorname{Hom}_{H_{\eta}}(\pi, 1)$ is nonzero. Here, $\operatorname{Hom}_{H_{\eta}}(\pi, 1)$ designates the space of all linear forms $\Lambda$ on $V_{\pi}$ such that $\Lambda(\pi(h) v)=\Lambda(v)$, for all $h \in H_{\eta}$ and all $v \in V_{\pi}$.

LEMMA 3.1. If $\pi$ is a complex representation of $G$ then for each $g \in G$ the map $\Lambda \mapsto \Lambda_{g}$, where $\Lambda_{g}(v)=\Lambda\left(\pi(g)^{-1} v\right)$ defines an isomorphism $\operatorname{Hom}_{H_{\eta}}(\pi, 1) \cong$ $\operatorname{Hom}_{g H_{\eta} g^{-1}}(\pi, 1)$.

Since our objective in this paper is to analyze the spaces $\operatorname{Hom}_{H_{\eta}}(\pi, 1)$ associated to a given irreducible, tame supercuspidal representation $\pi$, Lemma 3.1 implies that only the conjugacy class of $H_{\eta}$ is relevant. Therefore, we are justified in making the following simplifying assumption in the rest of the paper: 
ASSUMPTION. If $n$ is odd, we assume $\eta=1$. If $n$ is even, we assume that either $\eta=1$ or $\eta$ is some fixed element $\alpha \in E^{\prime \times}$ such that $N_{E^{\prime} / F^{\prime}}(\alpha) \notin N_{F / F^{\prime}}\left(F^{\times}\right)$. Furthermore, if $\eta \neq 1$ and $E / E^{\prime}$ is unramified, we assume $\eta$ is a prime element in $E^{\prime}$. If $\eta \neq 1$ and $E / E^{\prime}$ is ramified, we assume $\eta$ is a nonsquare root of unity in $E^{\prime}$.

The existence of $\alpha$ is guaranteed by Lemma 2.2. The fact that, when $\eta \neq 1$, the specific choices of $\eta$ are consistent with the condition which defines $\alpha$ is apparent from the constructions in Case 1 and 3 in the proof of Lemma 2.2. It is also apparent from examining this proof that the prime element in $E^{\prime}$ or nonsquare root of unity may be chosen arbitrarily. This will be relevant at times when we wish to place compatibility restrictions on the choices of prime elements. The fact that $\eta$ is hermitian results from our choice of embedding of $E$ in $g$ in the previous section.

Having fixed $\eta$, we let $H=H_{\eta}$. Then $H$ may regarded as the group of elements $h \in G$ such that $\sigma_{\eta}(h)=h^{-1}$, where $\sigma_{\eta}$ is the anti-involution of $g$ (and, by restriction, $G)$ given by $\sigma_{\eta}(x)=\eta x^{*} \eta^{-1}$. The restriction of $\pi$ to the center $Z$ of $G$ is a one-dimensional representation $\omega: Z \rightarrow \mathbb{C}^{\times}$which, if $\pi$ is $H_{\eta}$-distinguished, must be trivial on the kernel of $N_{F / F^{\prime}}: F^{\times} \rightarrow F^{\prime \times}$. Therefore, we will fix, once and for all, such an $\omega$ and only consider those $\pi$ which restrict to this $\omega$. Equivalently, we have fixed a homomorphism $\omega_{0}: N_{F / F^{\prime}}\left(F^{\times}\right) \rightarrow \mathbb{C}^{\times}$and then let $\omega=\omega_{0} \circ N_{F / F^{\prime}}$.

Let us now turn our attention towards the similitude groups. Most of the above remarks regarding unitary groups apply equally well to unitary similitude groups, though we will not repeat them. The homomorphism $\mu_{\eta}$, referred to as the 'similitude ratio', gives rise to an exact sequence

$$
1 \rightarrow H_{\eta} \rightarrow H_{\eta}^{\prime} \rightarrow \mu_{\eta}\left(H_{\eta}^{\prime}\right) \rightarrow 1
$$

We observe that $Z H_{\eta}$ consists of those $g \in H_{\eta}^{\prime}$ such that $\mu_{\eta}(g)$ lies in the image of the norm map $N_{F / F^{\prime}}: F^{\times} \rightarrow F^{\prime \times}$. In other words, we have another exact sequence

$$
1 \rightarrow Z H_{\eta} \rightarrow H_{\eta}^{\prime} \rightarrow \mu_{\eta}\left(H_{\eta}^{\prime}\right) / N_{F / F^{\prime}}\left(F^{\times}\right) \rightarrow 1 .
$$

It follows that $H_{\eta}^{\prime} / Z H_{\eta}$ is a finite abelian group isomorphic to $\mu_{\eta}\left(H_{\eta}^{\prime}\right) / N_{F / F^{\prime}}\left(F^{\times}\right)$.

Fix a homomorphism $\chi: H_{\eta}^{\prime} \rightarrow \mathbb{C}^{\times}$which restricts to $\omega$ on $Z$ and is trivial on $H_{\eta}$. This is equivalent to selecting a homomorphism $\chi_{0}: \mu_{\eta}\left(H_{\eta}^{\prime}\right) \rightarrow \mathbb{C}^{\times}$which restricts to $\omega_{0}$ on $N_{F / F^{\prime}}\left(F^{\times}\right)$and then taking $\chi=\chi_{0} \circ \mu_{\eta}$. The number of such homomorphisms is the same as the order of $\mu_{\eta}\left(H_{\eta}^{\prime}\right) / N_{F / F^{\prime}}\left(F^{\times}\right)$. Let $\operatorname{Hom}_{H_{\eta}^{\prime}}(\pi, \chi)$ denote the space of all linear forms $\Lambda$ on $V_{\pi}$ such that $\Lambda(\pi(g) v)=\chi(g) \Lambda(v)$, for all $g \in H_{\eta}^{\prime}$ and $v \in V_{\pi}$. We say $\pi$ is $\left(H_{\eta}^{\prime}, \chi\right)$-distinguished if $\operatorname{Hom}_{H_{\eta}^{\prime}}(\pi, \chi) \neq 0$. Fourier inversion on the finite group $H_{\eta}^{\prime} / Z H_{\eta}$ yields the following:

LEMMA 3.2. If $\pi$ is a complex representation of $G$ which restricts to $\omega$ on $Z$ then there is a canonical isomorphism $\operatorname{Hom}_{H_{\eta}}(\pi, 1) \cong \oplus_{\chi} \operatorname{Hom}_{H_{\eta}^{\prime}}(\pi, \chi)$, where $\chi$ ranges over the homomorphisms $H_{\eta}^{\prime} \rightarrow \mathbb{C}^{\times}$which restrict to $\omega$ on $Z^{\prime \prime}$ and are trivial on $H_{\eta}$. In particular, $\Lambda \in \operatorname{Hom}_{H_{\eta}}(\pi, 1)$ is mapped to $\left(\Lambda_{\chi}\right)$, where 


$$
\Lambda_{\chi}(v)=\sum_{g \in H_{\eta}^{\prime} / Z H_{\eta}} \Lambda(\pi(g) v) \chi(g)^{-1} .
$$

The inverse mapping is given by

$$
\Lambda(v)=\left|\mu_{\eta}\left(H_{\eta}^{\prime}\right) / N_{F / F^{\prime}}\left(F^{\times}\right)\right|^{-1} \sum_{\chi} \Lambda_{\chi}(v) .
$$

Lemma 3.2 exhibits the precise relation between distinguishedness relative to unitary groups and distinguishedness relative to unitary similitude groups. Before analyzing this relation, we now state one of our main results:

THEOREM 3.3. If $\pi$ is an irreducible tame supercuspidal representation of $G$ then the dimension of $\operatorname{Hom}_{H}(\pi, 1)$ is at most one.

The proof of Theorem 3.3 occupies the bulk of this paper. It is summarized in Section 14. For the remainder of this section, we assume Theorem 3.3 is true. Suppose $n$ is odd. Then $H_{\eta}^{\prime}=Z H_{\eta}$ and $\mu_{\eta}\left(H_{\eta}^{\prime}\right)=N_{F / F^{\prime}}\left(F^{\times}\right)$. If $\pi$ is $H$-distinguished then its central character $\omega$ will be a base change lift of two characters $\omega_{1}$ and $\omega_{2}$ of $F^{\prime \times}$. Both $\omega_{1}$ and $\omega_{2}$ restrict to the same quasicharacter $\chi$ of $N_{F / F^{\prime}}\left(F^{\times}\right)$. Lemma 3.2 reduces to the identity $\operatorname{Hom}_{H_{\eta}}(\pi, 1)=\operatorname{Hom}_{H_{\eta}^{\prime}}(\pi, \chi)$. In fact, when $n$ is odd then all notions of distinguishedness are equivalent. In other words, if $\pi$ is $H_{\eta}$-distinguished for any $\eta$ then it is $H_{\eta}$-distinguished for all $\eta$. Moreover, $\pi$ is $H_{\eta}$-distinguished if and only if it is $\left(H_{\eta}^{\prime}, \chi\right)$-distinguished.

Now suppose $n$ is even. Then $H_{\eta}^{\prime} / Z H_{\eta}$ has order two and $\mu_{\eta}\left(H_{\eta}^{\prime}\right)=F^{\prime x}$. Lemma 3.2 says

$$
\operatorname{Hom}_{H_{\eta}}(\pi, 1) \cong \operatorname{Hom}_{H_{\eta}^{\prime}}\left(\pi, \omega_{1} \circ \mu_{\eta}\right) \oplus \operatorname{Hom}_{H_{\eta}^{\prime}}\left(\pi, \omega_{2} \circ \mu_{\eta}\right) .
$$

Theorem 3.3 implies that we have the following dichotomy:

PROPOSITION 3.4. If $\pi$ is an $H_{\eta}$-distinguished irreducible tame supercuspidal representation of $G$ then it is either $\left(H_{\eta}^{\prime}, \omega_{1} \circ \mu\right)$-distinguished or $\left(H_{\eta}^{\prime}, \omega_{2} \circ \mu\right)$-distinguished, but not both.

\section{Cuspidal Representations Invariant Under an Automorphism}

There are corresponding theories over global fields and over finite fields of cuspidal representations of $\mathrm{GL}(n)$ which are distinguished by a unitary group. It is known, for example, that the analogue of Theorem 3.3 is true for such fields. We now recall two results for finite fields and then discuss their analogues for $p$-adic fields. The first result is based on analogous facts appearing in $[25,26]$.

LEMMA 4.1. Let $E$ be a degree $n$ extension of a finite field $F$ which is embedded, via an F-embedding, in the ring $M$ of $n$-by- $n$ matrices with entries in $F$. Assume 1 is a ring automorphism of $M$ and let $G=M^{\times}=\operatorname{GL}(n, F)$ and $T=E^{\times}$. Suppose $\theta$ is a regular character of $T$ and let $\kappa$ be the irreducible, cuspidal representation of $G$ associated to $\theta$ 
by the Deligne-Lusztig construction. Then $\kappa \sim \kappa \circ 1$ precisely when there exists an automorphism $\sigma$ of $E$ which agrees with $\imath$ on $F$ and satisfies $\theta=\theta \circ \sigma$. Moreover, if $\imath \mid F$ has order $k$, then $\sigma$ must also have order $k$.

Proof. The Deligne-Lusztig construction has an inherent symmetry under automorphisms which yields the identity:

$$
R_{l(T)}^{G}\left(\theta \circ l^{-1}\right) \circ \imath=R_{T}^{G}(\theta) .
$$

It follows that $\kappa \sim \kappa \circ \imath$ precisely when

$$
R_{l(T)}^{G}\left(\theta \circ l^{-1}\right)=R_{T}^{G}(\theta) .
$$

But the latter condition is equivalent to the existence of an $F$-isomorphism $\alpha: l(E) \rightarrow E$ which satisfies $\theta \circ l^{-1}=\theta \circ \alpha$. The existence of $\alpha$ is in turn equivalent to the existence of an automorphism $\sigma(=\alpha \circ \imath)$ of $E$ which agrees with $\imath$ on $F$ and satisfies $\theta=\theta \circ \sigma$.

Now let $k$ denote the order of $\imath|F=\sigma| F$. Let $E^{\prime}$ denote the fixed field of $\sigma^{k}$. Since $\theta=\theta \circ \sigma^{k}$, Hilbert's Theorem 90 implies that $\theta$ factors through the norm map from $E$ to $E^{\prime}$. Since $E^{\prime} \supset F$, regularity of $\theta$ implies that $E^{\prime}=E$. Thus $\sigma$ has order $k$.

Assume now that the restriction of $\imath$ to $F$ has order 2 and thus $\sigma$ must have order 2 . Let $E^{\prime}$ be the fixed field of $\sigma$ and put $F^{\prime}=E^{\prime} \cap F$. If $F^{\prime}$ has $q$ elements, then

$$
|F|=q^{2}, \quad\left|E^{\prime}\right|=q^{n} \quad \text { and } \quad|E|=q^{2 n} .
$$

There are two notable consequences of this. First, we observe $\sigma(t)=t^{q^{n}}$. Thus, $\kappa \sim \kappa \circ l$ if and only if $\theta(t)=\theta\left(t^{t^{n}}\right)$, for all $t \in T$. Equivalently, $\theta$ is trivial on $\mathrm{U}\left(1, E / E^{\prime}\right)$. Next, we note that $F$ is a subfield of $E^{\prime}$ exactly when $n$ is even. In this case, the condition $\theta=\theta \circ \sigma$ contradicts the regularity of $\theta$ and thus no such $\theta$ can exist. (One can also arrive at a contradiction by arguing that $E^{\prime}$ cannot contain $F$ since $\sigma|F=\imath| F$ is nontrivial.) We also remark that the field $E^{\prime}$ is independent of $\sigma$, since it is the unique subfield of $E$ of order $q^{n}$.

PROPOSITION 4.2. Let $E$ be a degree $n$ extension of a finite field $F$ which is embedded, via an F-embedding, in the ring $M$ of $n$-by-n matrices with entries in $F$. Assume that $F$ is a quadratic extension of some field $F^{\prime}$ and $l$ is the automorphism of $M$ given by applying the nontrivial Galois automorphism of $F / F^{\prime}$ to the entries of each matrix in $M$. Let $G=M^{\times}=\mathrm{GL}(n, F)$ and $T=E^{\times}$. Suppose $\theta$ is a regular character of $T$ and let $\kappa$ be the irreducible, cuspidal representation of $G$ associated to $\theta$ by the Deligne-Lusztig construction. Then the following conditions are equivalent:

(i) The space of $\kappa$ has nonzero vectors fixed by $\mathrm{U}\left(n, F / F^{\prime}\right)$.

(ii) $\kappa \sim \kappa \circ l$.

(iii) There exists an automorphism $\sigma$ of $E$ which agrees with $l$ on $F$ and satisfies $\theta=\theta \circ \sigma$.

(iv) $\theta$ is trivial $\mathrm{U}\left(1, E / E^{\prime}\right)$.

When $n$ is even, these conditions are never satisfied. 
Proof. The equivalence of conditions (i) and (ii) is a result of Gow [7]. The equivalence of conditions (ii), (iii) and (iv) follows from the above discussion.

The fact that conditions in Proposition 4.2 cannot be satisfied when $n$ is even will allow us to strengthen the results of [9] in Proposition 12.2 below. There is an analogue of Lemma 4.1 for supercuspidal representations:

LEMMA 4.3. Let $p$ be an odd prime and $F$ a finite extension of $Q_{p}$. Let $E$ be a tamely ramified degree $n$ extension of $F$ which is embedded, via an $F$-embedding, in the ring $M$ of $n$-by-n matrices with entries in $F$. Assume $l$ is a ring automorphism of $M$ and let $G=M^{\times}=\mathrm{GL}(n, F)$ and $T=E^{\times}$. Suppose $\theta$ is an admissible character of $T$ and let $\pi$ be the irreducible, supercuspidal representation of $G$ associated to $\theta$ by Howe's construction. Then $\pi \sim \pi \circ \mathrm{l}$ precisely when there exists an automorphism $\sigma$ of $E$ which agrees with $\imath$ on $F$ and satisfies $\theta=\theta \circ \sigma$. Moreover, if $\imath \mid F$ has order $k$, then $\sigma$ must also have order $k$.

Proof. The proof of Lemma 4.3 is entirely analogous to the proof of Lemma 4.1 since Howe's construction has the same type of symmetry under automorphisms as the Deligne-Lusztig construction.

We now would like to consider the analogue of Proposition 4.2 for supercuspidal representations.

THEOREM 4.4. Let $p$ be an odd prime and $F$ a finite extension of $\mathrm{Q}_{p}$. Let E be a tamely ramified degree $n$ extension of $F$ which is embedded, via an F-embedding, in the ring $M$ of $n$-by- $n$ matrices with entries in $F$. Assume that $F$ is a quadratic extension of some field $F^{\prime}$ and $l$ is the automorphism of $M$ given by applying the nontrivial Galois automorphism of $F / F^{\prime}$ to the entries of each matrix in $M$. Let $G=M^{\times}=\operatorname{GL}(n, F)$ and $T=E^{\times}$. Suppose $\theta$ is an admissible character of $T$ and let $\pi$ be the irreducible, supercuspidal representation of $G$ associated to $\theta$ by Howe's construction. Let $H$ be a unitary group in $G$ associated to some Hermitian matrix $\eta \in G$. Then the following conditions are equivalent:

(i) The space $\operatorname{Hom}_{H}(\pi, 1)$ is nonzero.

(ii) $\pi \sim \pi \circ l$.

(iii) $\pi$ is a base change lift from $\mathrm{GL}\left(n, F^{\prime}\right)$.

(iv) There exists an automorphism $\sigma$ of $E$ which agrees with 1 on $F$ and satisfies $\theta=\theta \circ \sigma$.

(v) $\theta$ is trivial $\mathrm{U}\left(1, E / E^{\prime}\right)$, where $E^{\prime}$ is the fixed field of an automorphism of $E$ of order two which agrees with 1 on $F$.

The equivalence of (ii), (iv) and (v) follows from Lemma 4.3.

DEFINITION. Under the hypotheses of Theorem 4.4, a representation $\pi$ which satisfies condition (ii) will be referred to as a Galois invariant representation. 
The equivalence of (ii) and (iii) is part of the theory of quadratic base change. We recall that, by definition, if $\pi$ is a base change lift of $\pi^{\prime}$ then this means that there is a specific relation between the twisted character of $\pi$ and the character of $\pi^{\prime}$. For more details on base change, we refer the reader to [2], in particular, Theorem 6.2. The fact that (i) implies (ii) is addressed in the next section. The proof that (ii) implies (i) uses the theory developed in the bulk of this paper. A summary of the proof is in Section 14.

It should be noted that, due to Theorem 3.3, condition (i) in the statement of Theorem 4.4 is equivalent to the condition $\operatorname{dim} \operatorname{Hom}_{H}(\pi, 1)=1$. We also remark that in condition (v) $E^{\prime}$ cannot contain $F$ since $\sigma|F=\imath| F$ is nontrivial. Since the five conditions in Theorem 4.4 do not depend on the choice of the Hermitian matrix $\eta$, we have the following corollary:

COROLLARY 4.5. Let $p$ be an odd prime and $F / F^{\prime}$ a quadratic extension, where $F^{\prime}$ is a finite extension of $Q_{p}$. Let $\pi$ be an irreducible tame supercuspidal representation of $G=\mathrm{GL}(n, F)$. Suppose $H$ and $H^{\prime}$ are unitary groups in $G$ associated to two matrices in $G$ which are Hermitian with respect to $F / F^{\prime}$. Then $\pi$ is $H$-distinguished if and only if it is $H^{\prime}$-distinguished.

Let us now mention another issue regarding quadratic base change for supercuspidal representations which is not resolved in the present paper. Suppose $E / F$ is a tamely ramified degree $n$ extension and $\theta$ is a quasicharacter of $E^{\times}$which is admissible over $F$. Let $\pi_{\theta}$ denote the irreducible, supercuspidal representation of $G$ given by Howe's construction [15] and suppose $\pi_{\theta}$ is a quadratic base change lift from $G^{\prime}=\mathrm{GL}\left(n, F^{\prime}\right)$. Then, in fact, $\pi$ is the base change lift of two irreducible, supercuspidal representations $\pi^{\prime}$ and $\pi^{\prime \prime}$ of $G^{\prime}=\operatorname{GL}\left(n, F^{\prime}\right)$. According to Theorem 4.4, there must exist an automorphism $\sigma$ of order two of $E$ which restricts to the nontrivial Galois automorphism of $F / F^{\prime}$ and which satisfies $\theta=\theta \circ \sigma$. Thus $\theta$ is a base change lift of two quasicharacters $\theta^{\prime}$ and $\theta^{\prime \prime}$ of the multiplicative group of the fixed field $E^{\prime}$ of $\sigma$. We can order the latter quasicharacters so that $\theta^{\prime}$ restricts to the central character of $\pi^{\prime}$. It is tempting to conjecture that $\pi^{\prime}=\pi_{\theta^{\prime}}$ and $\pi^{\prime \prime}=\pi_{\theta^{\prime \prime}}$. However, as we will see in this paper, there is more than one 'natural' correspondence between quasicharacters and tame supercuspidal representations. For any one of these correspondences, one might expect that these identities are true up to twisting by a quasicharacter which, hopefully, can be explicitly computed.

\section{Galois Invariance of Distinguished Supercuspidal Representations}

The purpose of this section is to state the following result (which is proven in [10]) and to briefly indicate the (global) techniques involved in its proof.

PROPOSITION 5.1. Assume $F / F^{\prime}$ is a quadratic extension of non-Archimedean local fields of characteristic zero with residue fields of odd characteristic. Assume $\eta \in \mathrm{GL}(n, F)$ is Hermitian with respect to $F / F^{\prime}$. If $\pi$ is an irreducible, supercuspidal 
representation of $\operatorname{GL}(n, F)$ which is distinguished with respect to the unitary group $H_{\eta}$ then $\pi$ must be Galois invariant.

For the rest of this section, we will switch to global notations. Let $F / F^{\prime}$ be a quadratic extension of number fields. Our attention will be focused on a particular finite place $v_{0}$ of $F^{\prime}$ which is inert in $F$. Let $w_{0}$ be the place of $F$ which lies above $v_{0}$. The quadratic extension which occurs in Proposition 5.1 should be viewed as the quadratic extension $F_{w_{0}} / F_{v_{0}}^{\prime}$. We consider the $F^{\prime}$-group $\mathbf{G}$ which is obtained from the $F$-group $\mathrm{GL}_{n}$ by restriction of scalars. Let $G=\mathbf{G}\left(F^{\prime}\right)=\mathrm{GL}(n, F)$, $G_{\mathrm{A}}=\mathbf{G}\left(F_{\mathrm{A}}^{\prime}\right)=\mathrm{GL}\left(n, F_{\mathrm{A}}\right)$ and, when $v$ is place of $F^{\prime}$, let $G_{v}=\mathbf{G}\left(F_{v}^{\prime}\right)$. We use similar notations for the other $F^{\prime}$-groups we consider. Fix an automorphism $\alpha$ of $\mathbf{G}$ of order two which is defined over $F^{\prime}$ and let $\mathbf{H}$ be the $F^{\prime}$-subgroup of $\mathbf{G}$ consisting of the fixed points of $\alpha$. Let $\mathbf{Z}$ be the center of $\mathbf{G}$ and let $\mathbf{Z}_{\mathbf{H}}=\mathbf{Z} \cap \mathbf{H}$.

Suppose $\omega=\otimes_{v} \omega_{v}$ is a character of $Z_{\mathrm{A}}$ which is trivial on $Z_{H, \mathrm{~A}} Z$ and let $\pi$ be an irreducible, automorphic, cuspidal $H$-distinguished representation of $G_{\mathrm{A}}$ with central character $\omega$. We say that $\pi$ is H-distinguished if there exists $\varphi$ in the space of $\pi$ such that

$$
P(\varphi)=\int_{Z_{H, \mathrm{~A}} H \backslash H_{\mathrm{A}}} \varphi(h) \mathrm{d} h \neq 0 .
$$

The following is proven in [10]:

THEOREM 5.2. If $\rho$ is an $H_{v_{0}}$-distinguished, irreducible, supercuspidal representation of $G_{v_{0}}=\mathrm{GL}\left(n, F_{w_{0}}\right)$ then there exists an H-distinguished, irreducible, automorphic, cuspidal representation $\pi=\otimes_{v} \pi_{v}$ of $G_{\mathrm{A}}=\mathrm{GL}\left(n, F_{\mathrm{A}}\right)$ such that $\pi_{v_{0}} \simeq \rho$.

For our purposes, the automorphism $\alpha$ is given by $\alpha(g)=\eta^{t} \bar{g}^{-1} \eta^{-1}$, where $\eta$ is a fixed Hermitian matrix in $\operatorname{GL}(n, F)$. and let $U(\eta)$ be the associated unitary subgroup of $\operatorname{GL}(n, F)$. The statement of Proposition 5.1 involves the choice of a Hermitian matrix in $\operatorname{GL}\left(n, F_{w_{0}}\right)$. For simplicity, we assume that this matrix lies in $\operatorname{GL}(n, F)$ and corresponds to our matrix $\eta$. (It is explained in [10] how one easily reduces to this case.) Let $\rho$ be the representation which occurs in the hypothesis of Proposition 5.1. Applying Theorem 5.2, we obtain an automorphic cuspidal representation $\pi$ with $\rho$ as the component at $v_{0}$. Now an elementary argument given in [13], and also appearing in $[5,10]$ and [19], proves that $\pi$ is Galois invariant in the sense that $\pi(g)$ is equivalent to the representation $g \mapsto \pi(\bar{g})$, where $g \mapsto \bar{g}$ is the nontrivial Galois automorphism of $F / F^{\prime}$. Therefore $\rho$ must also be Galois invariant and Proposition 5.1 follows.

\section{Admissible Quasicharacters}

We will now recall some basic facts about admissible quasicharacters from [15] and refine various details so as to make them compatible with the relevant Galois automorphisms which arise in our applications. 
First, we need some notations. Let $E$ be a tamely ramified extension of $F$ of degree $n$ or, in other words, if $p$ is the characteristic of the residue field of $E$ and if $e(E / F)$ is the ramified degree of $E / F$ then $p$ does not divide $e(E / F)$. Whenever $L$ is a subfield of $E$, we let $\mathfrak{S}_{L}$ denote the ring of integers of $L$ and $\mathfrak{P}_{L}$ is the maximal ideal of $\mathfrak{D}_{L}$. The residue field is $k_{L}=\mathfrak{D}_{L} / \mathfrak{P}_{L}$ and its order is denoted $q_{L}$. Choose a prime element $\varpi_{L}$ for each $L$ so that if $F \subseteq L_{1} \subseteq L_{2} \subseteq E$ then $\varpi_{\left.L_{2} / L_{1}\right)} \varpi_{L_{1}}^{-1}$ is a root of unity in $L_{1}$ whose order is relatively prime to $p$. Then if $C_{L}$ is the group generated by $\varpi_{L}$ and the roots of unity in $L$ with order relatively prime to $p$ then $N_{L_{2} / L_{1}}\left(C_{L_{2}}\right) \subset C_{L_{1}}$.

Howe's construction depends on the choice of a quasicharacter $\theta$ of $E^{\times}$which is admissible over $F$ in the following sense.

DEFINITION. A quasicharacter $\theta$ of $E^{\times}$is admissible over $F$ if:

(1) $\theta \neq \theta^{\prime} \circ N_{E / L}$ for every quasicharacter $\theta^{\prime}$ of every intermediate field $L$ of $E / F$ (other than $E$ ), and

(2) if $\theta=\theta^{\prime} \circ N_{E / L}$ on $1+\mathfrak{B}_{E}$ for some quasicharacter $\theta^{\prime}$ of some intermediate field $L$ of $E / F$ (other than $E$ ), then $E / L$ must be unramified.

We will also say that $\theta$ is a base change lift from $L$ if $\theta=\theta^{\prime} \circ N_{E / L}$ for some quasicharacter $\theta^{\prime}$ of $L$. In this case, we will abbreviate $\theta^{\prime} \circ N_{E / L}$ as $\theta^{\prime}{ }^{E}$. The admissible quasicharacters of interest to us are those which are quadratic base change lifts. In other words, we will assume that $E$ is a quadratic extension of a field $E^{\prime}$ and $\theta=\theta^{\prime} E$ for some quasicharacter $\theta^{\prime}$ of $E^{\prime \times}$. We will let $\sigma$ denote the nontrivial automorphism of $E / E^{\prime}$. Our assumption that $\theta$ is admissible over $F$ implies that $F$ cannot be contained in $E^{\prime}$. Thus $F$ must be a quadratic extension of the field $F^{\prime}=F \cap E^{\prime}$.

The most basic examples of admissible quasicharacters are those quasicharacters $\theta$ of $E^{\times}$which are generic over $F$. To recall the notion of genericity, we set some more notations. Fix a character $\psi_{F^{\prime}}$ of $F^{\prime}$ of conductor $\mathfrak{B}_{F^{\prime}}$. For each intermediate field $L$ of $E / F^{\prime}$, let $\psi_{L}$ be the character of $L$ of conductor $\mathfrak{B}_{L}$ defined by $\psi_{L}=\psi_{F} \circ \operatorname{tr}_{E / L}$. The conductoral exponent of $\theta$ is denoted $f(\theta)$. If $f(\theta)>1$ then there exists a unique element $\gamma_{\theta} \in C_{E}$ such that $\theta(1+x)=\psi_{E}\left(\gamma_{\theta} x\right)$, for all $x \in \mathfrak{P}_{E}^{f(\theta)-1}$. We refer to $\gamma_{\theta}$ as the standard representative for $\theta$. The standard representatives are invariant under base change in the sense that $\gamma_{\theta}=\gamma_{\theta_{0}}$ when $\theta$ is a base change lift of $\theta_{0}$.

DEFINITION. A quasicharacter $\theta$ of $E^{\times}$is generic over $F$ if $f(\theta)>1$ and $E=F\left[\gamma_{\theta}\right]$, or if $f(\theta)=1$ and $\theta$ is admissible over $F$.

Note that if $f(\theta)>1$, then invariance of standard representatives under base change implies that $\theta$ must be admissible over $F$ if it is generic over $F$ and $f(\theta)>1$. We also emphasize that if $f(\theta)=1$, then $\gamma_{\theta}$ is not defined.

In Section 2, we discussed towers of intermediate fields of $E / F$. The towers of interest to us arise from the following factorization: 
THE HOWE FACTORIZATION OF $\theta$. Given a quasicharacter $\theta$ of $E^{\times}$which is admissible over $F$, there is a unique tower $F=E_{0} \subset E_{1} \subset \cdots \subset E_{r}=E$ and unique positive integers $f_{1}>\cdots>f_{r}$ such that there exists a (nonunique) sequence $\theta_{0}, \ldots, \theta_{r}$ of quasicharacters of $E_{0}^{\times}, \ldots, E_{r}^{\times}$, respectively, such that $\theta=\prod_{i=0}^{r} \theta_{i}^{E}$ and, when $i>0$, the conductoral exponent of $\theta_{i}^{E}$ is $f_{i}$ and, in addition, $\theta_{i}$ is generic over $E_{i-1}$.

We are specifically interested in those $\theta$ which are base change lifts from $E^{\prime}$. For these quasicharacters, we have:

PROPOSITION 6.1. Assume $\theta$ is a quasicharacter of $E^{\times}$which is admissible over $F$ and suppose $\theta^{\prime}$ is a quasicharacter of $E^{\prime \times}$ such that $\theta=\theta^{\prime}$. Then $\theta^{\prime}$ is admissible over $F^{\prime}$. Assume $\theta^{\prime}=\prod_{i=0}^{r} \theta_{i}^{\prime} E^{\prime}$ is a Howe factorization of $\theta^{\prime}$ and $F^{\prime}=E^{\prime}{ }_{0} \subset E^{\prime}{ }_{1} \subset$ $\cdots \subset E^{\prime}{ }_{r}=E^{\prime}$ is the corresponding tower of fields. Let $E_{i}=F E^{\prime}{ }_{i}$, for $0 \leqslant i \leqslant r$, and let $\theta_{i}$ be the quasicharacter of $E_{i}^{\times}$defined by $\theta_{i}^{\prime} E_{i}$. Then $\theta=\prod_{i=0}^{r} \theta_{i}^{E}$ is a Howe factorization of $\theta$.

Proof. Suppose that $\theta^{\prime}$ is not admissible over $F^{\prime}$. There are two ways this could happen. In the first case, there exists a proper subextension $L^{\prime}$ of $E^{\prime} / F^{\prime}$ such that $\theta^{\prime}=\theta_{L^{\prime}}^{\prime E^{\prime}}$ for some quasicharacter $\theta_{L^{\prime}}^{\prime}$ of $L^{\prime \times}$. But then if $L=F L^{\prime}$ and $\theta_{L}=\theta_{L^{\prime}}^{\prime}$, we would have $\theta=\theta_{L}^{E}$ contradicting the admissibility of $\theta$. In the second case, there exists a proper subextension $L^{\prime}$ of $E^{\prime} / F^{\prime}$ such that $E^{\prime} / L^{\prime}$ is ramified and $\theta^{\prime}=\theta_{L^{\prime}}^{E^{\prime}}$ on $1+\mathfrak{B}_{E^{\prime}}$ for some quasicharacter $\theta_{L^{\prime}}^{\prime}$ of $L^{\prime \times}$. Note that $N_{E / E^{\prime}}\left(1+\mathfrak{B}_{E}\right)=1+\mathfrak{P}_{E^{\prime}}$ and therefore if $L=F L^{\prime}$ and $\theta_{L}=\theta_{L^{\prime}}^{L}$ then $\theta=\theta_{L}^{E}$ on $1+\Re_{E}$. If $E / L$ is ramified this contradicts the admissibility of $\theta$. On the other hand, if $E / L$ is unramified then $L / L^{\prime}$ must be ramified and $E / E^{\prime}$ must be unramified and hence generated by a square root of a root of unity $\epsilon$. Replacing $L$ by $L^{\prime}[\epsilon]$ causes $L / L^{\prime}$ to become unramified and $E / L$ to become ramified. Again, we contradict the admissibility of $\theta$ and deduce that $\theta^{\prime}$ must indeed be admissible over $F^{\prime}$.

Assume $i>0$ and $f_{i}>1$. By invariance of standard representatives under base change, we have $\gamma_{\theta_{i}}=\gamma_{\theta_{i}^{\prime}}$. Thus

$$
E_{i-1}\left[\gamma_{\theta_{i}}\right]=F E^{\prime}{ }_{i-1}\left[\gamma_{\theta_{i}}\right]=F E^{\prime}{ }_{i}=E_{i}
$$

and, consequently, $\theta_{i}$ is generic over $E_{i-1}$. Now suppose $i>0$ and $f_{i}=1$. Then since $N_{E / E^{\prime}}\left(1+\mathfrak{P}_{E}\right)=1+\mathfrak{S}_{E^{\prime}}$, we see that $\theta_{i}^{\prime}$ has conductor $1+\mathfrak{B}_{E^{\prime}}$. Therefore, $i=r$ and $E^{\prime}{ }_{i} / E^{\prime}{ }_{i-1}=E^{\prime} / E^{\prime}{ }_{r-1}$ is unramified and $\theta_{r}^{\prime}$ is not a base change lift from a proper intermediate field of $E^{\prime} / E_{r-1}^{\prime}$. The fact that $E^{\prime} / E^{\prime}{ }_{r-1}$ is unramified is equivalent to the fact that this extension must be generated by roots of unity of prime to $p$ order. Since $E / E_{r-1}$ inherits the latter property, it must also be unramified. Therefore, showing that $\theta_{r}$ is generic over $E_{r-1}$ amounts to showing it is not a base change lift from a proper intermediate field $L$ of $E / E_{r-1}$. However, if $\theta_{r}$ were a base change lift from $L$ then $\theta$ would also be a base change lift from $L$, which would contradict the admissibility of $\theta$. The proof is now complete.

We now record some elementary properties of the conductoral exponents of $\theta_{i}^{E}$ and $\theta_{i}^{\prime} E^{\prime}$. 
LEMMA 6.2. Let $f_{i}$ and $f_{i}^{\prime}$ be the conductoral exponents of $\theta_{i}^{E}$ and $\theta_{i}^{\prime} E^{\prime}$, respectively, when $1 \leqslant i \leqslant r$. Then:

(i) $f_{i}-1=e\left(E / E^{\prime}\right)\left(f_{i}^{\prime}-1\right)$, for all $i$.

(ii) $f_{r}=1$ if and only if $f_{r}^{\prime}=1$.

(iii) If $E / E^{\prime}$ is unramified then $f_{i}=f_{i}^{\prime}$, for all $i$.

(iv) If $E / E^{\prime}$ is ramified then $f_{i}$ is odd, for all $i$.

(v) If $f_{r}=1$ then $E / E^{\prime}$ is unramified if and only if $E_{r-1} / E_{r-1}^{\prime}$ is unramified.

(vi) If $f_{r}=1$ and $E / E^{\prime}$ is unramified then $n_{r-1}=\left[E: E_{r-1}\right]=\left[E^{\prime}: E_{r-1}^{\prime}\right]$ is odd.

Proof. In general, if $L / K$ is an extension of subfields of $E$ with ramified degree $e(L / K)$ and $\chi$ is a quasicharacter of $K^{\times}$then the relation between the conductoral exponents of $\chi$ and $\chi^{L}$ is given by

$$
f\left(\chi^{L}\right)-1=e(L / K)(f(\chi)-1) .
$$

Applying this to the conductoral exponents $f_{i}^{\prime}$ and $f_{i}$ gives (i). Assertions (ii), (iii) and (iv) are immediate consequences of (i). Assertion (v) holds, since $f_{r}=f_{r}^{\prime}=1$ and admissibility imply that $E / E_{r-1}$ and $E^{\prime} / E_{r-1}^{\prime}$ are unramified and thus $e\left(E / E^{\prime}\right)=$ $e\left(E / E^{\prime}{ }_{r-1}\right)=e\left(E_{r-1} / E_{r-1}^{\prime}\right)$. To prove (vi), let us suppose that $f_{r}=1, E / E^{\prime}$ is unramified and $n_{r-1}$ is even. Then $E^{\prime} / E^{\prime}{ }_{r-1}$ must contain a quadratic unramified extension of $E_{r-1}^{\prime}$. However, such a quadratic extension cannot exist since $E_{r-1}$ is the unique quadratic unramified extension of $E_{r-1}^{\prime}$ which is contained in $E$ and $E_{r-1}$ is not contained in $E^{\prime}$.

\section{Heisenberg and Weil Representations over $\mathbb{F}_{p}$}

Fix an odd prime $p$ and a nontrivial additive character $\zeta$ of $\mathbb{F}_{p}$. Let $W$ be a finitedimensional nondegenerate symplectic space over $\mathbb{F}_{p}$ with symplectic form $\langle\langle\rangle$,$\rangle .$ Assume that $W^{+}$and $W^{-}$form a polarization of $W$, that is, $W^{+}$and $W^{-}$are totally isotropic subspaces of $W$ and $W^{+}+W^{-}=W$. Let $\mathcal{Z}=\mathbb{F}_{p}$ and define a Heisenberg group structure on the Cartesian product $\mathcal{H}=W \times \mathcal{Z}$ by the multiplication rule

$$
\left(w_{1}, z_{1}\right)\left(w_{2}, z_{2}\right)=\left(w_{1}+w_{2}, z_{1}+z_{2}+\frac{1}{2}\left\langle\left\langle w_{1}, w_{2}\right\rangle\right\rangle\right) .
$$

We identify $\mathcal{Z}, W^{+}$and $W^{-}$with the subgroups $0 \times \mathcal{Z}, W^{+} \times 0$ and $W^{-} \times 0$, respectively.

Let $\zeta^{+}$be the character of $W^{+} \mathcal{Z}=W^{+} \times \mathcal{Z}$ defined by $\zeta^{+}(w z)=\zeta(z)$, where $w \in W^{+}$and $z \in \mathcal{Z}$. Let $(\rho, V)$ be the induced representation $\operatorname{Ind}_{W^{+} \mathcal{Z}}^{\mathcal{H}}\left(\zeta^{+}\right)$. This is a Heisenberg representation of $\mathcal{H}$ corresponding to the central character $\zeta$. The properties of such Heisenberg representations, as well as the associated Weil representations, are studied in detail in [6]. In this section, we recall some basic facts from [6] and develop the theory of invariant linear forms and distinguishedness for the Heisenberg representations. This theory is then applied in Section 8 to the construction of tame supercuspidal representations. 
If $\mathcal{K}$ is a subgroup of $\mathcal{H}$ then let

$$
V^{\mathcal{K}}=\{v \in V: \rho(k) \varphi=\varphi \text { for all } k \in \mathcal{K}\} .
$$

If $x \in \mathcal{H}$, we let $V_{x}^{\mathcal{K}}$ denote the space of functions in $V^{\mathcal{K}}$ with support contained in $W^{+} \mathcal{Z} x \mathcal{K}$. Define an $\mathcal{H}$-invariant inner product on $V$ by

$$
\varphi_{1} \cdot \varphi_{2}=\sum_{w \in W^{-}} \varphi_{1}(w) \bar{\varphi}_{2}(w) .
$$

There is an isomorphism $V^{\mathcal{K}} \cong \operatorname{Hom}_{\mathcal{K}}(\rho, 1)$ given by associating to $\varphi_{0} \in V^{\mathcal{K}}$ the linear form $\lambda(\varphi)=\varphi \cdot \varphi_{0}$.

The following elementary result gives an exhaustive description of when the Heisenberg representation $\rho$ is $\mathcal{K}$-distinguished with respect to any subgroup $\mathcal{K}$ of $\mathcal{H}$. Though we only need to use this result in one of its simplest cases, we include the general statement because it highlights the exceptional simplicity of Heisenberg representations over $\mathbb{F}_{p}$.

LEMMA 7.1. Suppose $\mathcal{K}$ is a subgroup of $\mathcal{H}$ and $X$ is a set of representatives for the double coset space $W^{+} \mathcal{Z} \backslash \mathcal{H} / \mathcal{K}$. Then $V^{\mathcal{K}}=\oplus_{x \in X} V_{x}^{\mathcal{K}}$ and each space $V_{x}^{\mathcal{K}}$ has dimension one if $W^{+}\left(x \mathcal{K} x^{-1}\right) \cap \mathcal{Z}=\{1\}$ and dimension zero otherwise.

Proof. If $f \in V^{\mathcal{K}}$ and $x \in X$ then define $f_{x} \in V^{\mathcal{K}}$ by taking $f_{x}=f$ on $W^{+} \mathcal{Z} x \mathcal{K}$ and $f_{x} \equiv 0$ outside of $W^{+} \mathcal{Z} x \mathcal{K}$. We have $f=\sum_{x \in X} f_{x}$. Therefore, we have a direct sum decomposition $V^{\mathcal{K}}=\oplus_{x \in X} V_{x}^{\mathcal{K}}$. So in order to compute $V^{\mathcal{K}}$ it suffices to compute the spaces $V_{x}^{\mathcal{K}}$.

Fix $x \in X$. If $V_{x}^{\mathcal{K}}$ is nonzero then there is a unique element $\xi_{x} \in V_{x}^{\mathcal{K}}$ such that $\xi_{x}(x)=1$ and it is defined on $W^{+} \mathcal{Z} x \mathcal{K}$ by $\xi_{x}(w z x k)=\zeta(z)$, where $w \in W^{+}, z \in \mathcal{Z}$ and $k \in \mathcal{K}$. In this case, $V_{x}^{\mathcal{K}}$ must be the one-dimensional space spanned by $\xi_{x}$.

The space $V_{x}^{\mathcal{K}}$ will be nonzero precisely when the above formula for $\xi_{x}$ gives a welldefined function. In order for $\xi_{x}$ to be well-defined, we must have $\zeta\left(z_{1}\right)=\zeta\left(z_{2}\right)$ whenever $w_{1} z_{1} x k_{1}=w_{2} z_{2} x k_{2}$, with $w_{1}, w_{2} \in W^{+}, z_{1}, z_{2} \in \mathcal{Z}$ and $k_{1}, k_{2} \in \mathcal{K}$. Note that since $\zeta$ is a nontrivial character of a cyclic group of finite order, the kernel of $\zeta$ is trivial. Thus the condition $\zeta\left(z_{1}\right)=\zeta\left(z_{2}\right)$ is equivalent to the condition $z_{1}=z_{2}$. Letting

$$
w=w_{1}^{-1} w_{2}, \quad z=z_{1}^{-1} z_{2} \quad \text { and } \quad k=k_{2} k_{1}^{-1},
$$

we see that $V_{s}^{\mathcal{K}} \neq 0$ precisely when the following condition is satisfied: whenever $w \in W^{+}, z \in \mathcal{Z}, k \in \mathcal{K}$ and $w z x k=x$, it must be the case that $z=1$. Equivalently, the latter condition says that $W^{+}\left(x \mathcal{K} x^{-1}\right) \cap \mathcal{Z}=\{1\}$.

A matrix representation for $\mathcal{H}$ is obtained as follows. Choose a basis $e_{1}, \ldots, e_{2 \ell}$ of $W$ such that $e_{1}, \ldots, e_{\ell}$ is a basis of $W^{+}$and $e_{\ell+1}, \ldots, e_{2 \ell}$ is a basis of $W^{-}$. Use this basis to identify $W$ with $\mathbb{F}_{p}^{2 \ell}$, viewed as a space of column vectors. Let $j$ be the matrix of the symplectic form in the sense $\left\langle\left\langle w_{1}, w_{2}\right\rangle\right\rangle={ }^{t} w_{1} j w_{2}$. We will assume our basis is chosen so that $j$ takes the block matrix form $\left(\begin{array}{rr}0 & 1 \\ -1 & 0\end{array}\right)$, where the blocks have dimensions $\ell \times \ell$. 
Identify $(w, z) \in \mathcal{H}$ with the block matrix

$$
\left(\begin{array}{ccc}
1 & \frac{1}{2}^{t} w j & z \\
0 & 1 & w \\
0 & 0 & 1
\end{array}\right) \in \operatorname{GL}\left(2 \ell+2, \mathbb{F}_{p}\right)
$$

where the diagonal blocks are square matrices of rank $1,2 \ell$ and 1 , respectively. The group $\mathcal{S}=\mathrm{Sp}(W)$ acts on $\mathcal{H}$ by its action on the first factor of $W \times \mathcal{Z}$. In the matrix representation, $s \in \mathcal{S}$ acts according to

$$
\operatorname{Ad}\left(\left(\begin{array}{lll}
1 & 0 & 0 \\
0 & s & 0 \\
0 & 0 & 1
\end{array}\right)\right) .
$$

The group $\mathcal{S}$ also has a more traditional matrix representation as the group block matrices $s=\left(\begin{array}{l}a \\ c d \\ c\end{array}\right)$, with $\ell \times \ell$ blocks, such that ${ }^{t} s j s=j$. In the latter representation, $\mathcal{S}$ acts on $W$ by matrix multiplication $\left(\begin{array}{l}a b \\ c d\end{array}\right)\left(\begin{array}{l}w^{+} \\ w^{-}\end{array}\right)$. We will sometimes use the notation $s \cdot w$ for the action of $\mathcal{S}$ on $W$, in order to avoid potential conflicts of notation between the two different matrix representations of $\mathcal{S}$. We will use a similar notation $s \cdot h$ for the action of $\mathcal{S}$ on $\mathcal{H}$.

We are also interested in the semidirect product $\mathcal{S} \times \mathcal{H}$ with multiplication given by

$$
\left(s_{1}, h_{1}\right)\left(s_{2}, h_{2}\right)=\left(s_{1} s_{2},\left(s_{2}^{-1} \cdot h_{1}\right) h_{2}\right) .
$$

In the $\operatorname{GL}\left(2 \ell+2, \mathbb{F}_{p}\right)$ matrix representation, $\mathcal{S} \times \mathcal{H}$ corresponds to $\mathcal{S H}=\{s h: s \in \mathcal{S}$, $h \in \mathcal{H}$, with multiplication given by matrix multiplication.

The Heisenberg representation $(\rho, V)$ of $\mathcal{H}$ has a unique extension $(\hat{\rho}, V)$ to $\mathcal{S} \times \mathcal{H}$ which we refer to as the 'Weil representation of $\mathcal{S} \ltimes \mathcal{H}$ ' (relative to the choice of central character and polarization). The equivalence class of $\hat{\rho}$ only depends on the central character. The Weil representation may be described explicitly as follows. The Siegel parabolic subgroup of $\mathcal{S}$ is the group $\mathcal{P}=\left\{s \in \mathcal{S}: s \cdot W^{+} \subset W^{+}\right\}$. In the $\operatorname{GL}\left(2 \ell, \mathbb{F}_{p}\right)$ representation, $\mathcal{P}$ consists of the elements in $\mathcal{S}$ of the form $\left(\begin{array}{l}a b \\ 0\end{array}\right)$. To be even more explicit, each element of $\mathcal{P}$ has the form

$$
p(x, y)=\left(\begin{array}{cc}
y & 0 \\
0 & { }^{t} y^{-1}
\end{array}\right)\left(\begin{array}{cc}
1 & x \\
0 & 1
\end{array}\right),
$$

where ${ }^{t} x=x$. The unipotent radical $\mathcal{N}$ of $\mathcal{P}$ consists of those elements of the form $p(x, y)$ with $y=1$. Since $\mathcal{S}$ is generated by $\mathcal{P}$ and the element $j=\left(\begin{array}{rl}0 & 1 \\ -1 & 0\end{array}\right)$, the Weil representation on $\mathcal{S}$ is completely described by the action of $\mathcal{P}$ and $j$. The action of $\mathcal{P}$ on $V$ is given by

$$
\hat{\rho}(p(x, y)) \varphi(h)=(\operatorname{det} y)^{(p-1) / 2} \varphi\left(p(x, y)^{-1} \cdot h\right) .
$$

The action of $j$ is nearly a Fourier transform. More specifically, if $\varphi \in V$ and $w^{-} \in W^{-}$then

$$
\hat{\rho}(j) \varphi\left(w^{-}\right)=\epsilon p^{-\ell / 2} \sum_{w \in W^{-}} \varphi(w) \zeta\left(\left\langle\left\langle j w, w^{-}\right\rangle\right\rangle\right),
$$


where $\epsilon$ is the root of unity defined by

$$
\epsilon=(-1)^{\ell(p-1) / 2}\left(p^{-1 / 2} \sum_{t \in \mathbb{F}_{p}} \zeta\left(t^{2} / 2\right)\right)^{-\ell} .
$$

(See [6] for more details.) Since $\hat{\rho}(j) \varphi$ is determined by its restriction to $W^{-}$, the equation above fully determines the action of $\hat{\rho}(j)$ on $V$.

PROPOSITION 7.2. Suppose $W_{0}$ is maximal totally isotropic subspace of $W$ embedded as a subgroup in $\mathcal{H}$ via the map $w \mapsto(w, 0)$ from $W$ to $W \times \mathcal{Z}$. Then $\operatorname{Hom}_{W_{0}}(\rho, 1)$ has dimension one. In particular, $\operatorname{Hom}_{W^{-}}(\rho, 1)$ is the one-dimensional space spanned by the linear form $\lambda(\varphi)=\varphi(1)$.

Proof. We begin by treating the case of $W_{0}=W^{-}$by applying Lemma 7.1 with $\mathcal{K}=W^{-}$. Since $\mathcal{H}=W^{+} \mathcal{Z} W^{-}$, we may take the set $X$ in the statement of Lemma 7.1 to be $\{1\}$. Then $\operatorname{Hom}_{W^{-}}(\rho, 1) \cong V^{W^{-}} \cong V_{1}^{W^{-}}$and, moreover, $V_{1}^{W^{-}}$has dimension one, since $W^{+} W^{-} \cap \mathcal{Z}=\{1\}$. But the linear form defined by $\lambda(\varphi)=\varphi(1)$ is clearly a nonzero element of $\operatorname{Hom}_{W^{-}}(\rho, 1)$ and, hence, we must have $\operatorname{Hom}_{W^{-}}(\rho, 1)=\mathrm{C} \lambda$. This proves our claims when $W_{0}=W^{-}$. Now suppose $W_{0}$ is an arbitrary maximal totally isotropic subspace of $W$. Then there exists $s \in \mathcal{S}$ such that $s \cdot W_{0}=W^{-}$. It follows that $W_{0}$ and $W^{-}$are conjugate as subgroups of $\mathcal{S} \times \mathcal{H}$. Consequently, $\operatorname{Hom}_{W_{0}}(\hat{\rho}, 1) \cong \operatorname{Hom}_{W^{-}}(\hat{\rho}, 1)$. But

$$
\operatorname{Hom}_{W_{0}}(\hat{\rho}, 1)=\operatorname{Hom}_{W_{0}}(\rho, 1) \text { and } \operatorname{Hom}_{W^{-}}(\hat{\rho}, 1)=\operatorname{Hom}_{W^{-}}(\rho, 1)=\mathrm{C} \lambda .
$$

Therefore, $\operatorname{Hom}_{W_{0}}(\rho, 1)$ has dimension one.

\section{The Construction of $\boldsymbol{\kappa}_{i}$}

The purpose of this section is to construct some auxiliary representations which arise in Howe's construction of tame supercuspidal representations of $G$. Our tame supercuspidal representations will have the form $\pi=\operatorname{Ind}_{R}^{G}(\kappa)$, where the inducing representation is a tensor product $\kappa=\kappa_{0} \otimes \cdots \otimes \kappa_{r}$ of the character $\kappa_{0}=\theta_{0} \circ$ det with certain representations $\kappa_{i}: R \rightarrow \operatorname{Aut}\left(V_{i}\right)$, where $1 \leqslant i \leqslant r$. Each $\kappa_{i}$ corresponds to a factor $\theta_{i}$ in the Howe factorization of the admissible quasicharacter $\theta$. The problem of computing $H$-invariant linear forms on the space of $\pi$ will ultimately reduce via a factorization theory to the problem of computing the spaces $\operatorname{Hom}_{R \cap H}\left(\kappa_{i}, \vartheta_{i}\right)$ for certain quadratic characters $\vartheta_{i}$

We adopt the notations used in the statement of Proposition 6.1. In particular, there are quasicharacters $\theta$ and $\theta^{\prime}$ such that $\theta=\theta^{\prime} E$ and associated Howe factorizations and towers of fields. Associated to the tower $\left\{E_{i}\right\}$ are the Lie algebras $\left\{\mathfrak{g}_{i}\right\}$ and the groups $\left\{G_{i}\right\}$ defined in Section 2, as well as the parahoric algebras and groups $\mathfrak{b}$, $\mathfrak{b}_{j}, \mathfrak{b}_{j, i}, B, B_{j}$ and $B_{j, i}$. As in the statement of the Howe factorization, $f_{i}$ denotes the conductoral exponent $f\left(\theta_{i}^{E}\right)$. When $0 \leqslant i \leqslant r$, let 


$$
\ell_{i}=\left\lfloor\frac{f_{i}}{2}\right\rfloor \quad \text { and } \quad m_{i}=\left\lfloor\frac{f_{i}+1}{2}\right\rfloor .
$$

In general, we will let $\mathfrak{O}_{i}$ and $\mathfrak{O}_{i}^{\prime}$ denote the rings of integers of $E_{i}$ and $E^{\prime}{ }_{i}$, respectively, and fix prime elements $\varpi_{i}$ and $\varpi_{i}^{\prime}$ in these rings according to the conventions in Section 6. Let $\mathfrak{P}_{i}=\varpi_{i} \mathfrak{N}_{i}$ and $\mathfrak{p}_{i}=\varpi_{i}^{\prime} \mathfrak{O}_{i}^{\prime}$. Thus $\mathfrak{P}_{E}=\mathfrak{P}_{r}$ and $\mathfrak{P}_{E^{\prime}}=\mathfrak{p}_{r}$. The corresponding residue fields are denoted $k_{i}$ and $k_{i}^{\prime}$.

The representations $\kappa_{i}$ we define will be representations of the group

$$
R=E^{\times} B(\theta)=E^{\times} B\left(\theta_{r}\right) \cdots B\left(\theta_{1}\right),
$$

with $B\left(\theta_{i}\right)=B_{\ell_{i}, i-1}$ and $B(\theta)=B\left(\theta_{r}\right) \cdots B\left(\theta_{1}\right)$.

Fix, for the remainder of this section, an index $i \in\{1, \ldots, r\}$. The first case we discuss is the case in which $f_{i}=1$ and thus $i=r$. Then the fact that $\theta_{r}$ is generic over $E_{r-1}$ implies $E / E_{r-1}$ must be unramified. We observe that $\theta_{r}$ restricts to a character of the multiplicative group of the residue field $k_{r}$ of $E$. The constructions of Green and Deligne and Lusztig [3] associate to the latter character a cuspidal representation $\left(\rho_{r}, V_{r}\right)$ of $\mathrm{GL}\left(n_{r-1}, k_{r-1}\right)$. We regard $\rho_{r}$ as a representation of $R \cap B$, using the fact that $(R \cap B) /\left(R \cap B_{1}\right) \cong \mathrm{GL}\left(n_{r-1}, k_{r-1}\right)$. The group $R$ is generated by the elements of $R \cap B$ together with a prime element $\varpi_{r-1}$ of $E_{r-1}$. Define the representation $\kappa_{r}$ of $R$ on $V_{r}$ by the formula $\kappa_{r}\left(\varpi_{r-1}^{j} k\right)=\theta_{r}\left(\varpi_{r-1}\right)^{j} \rho_{r}(k)$, for all $j \in Z_{\text {and }} k \in R \cap B$.

For the rest of this section, we assume $f_{i}>1$. Under this assumption, $\kappa_{i}$ is produced using a Heisenberg/Weil representation construction which depends on the choice of a 'special isomorphism' (in the sense of [31], as described below). There is a particularly desirable choice of special isomorphism which is compatible with the anti-involution $\sigma_{\eta}$. This special isomorphism comes from an especially convenient choice of polarization of the relevant symplectic space. Since this special isomorphism and the associated correspondence between admissible quasicharacters and tame supercuspidal representations may not agree with the traditional conventions in the literature, it will be necessary in the next section to discuss some aspects of how varying the special isomorphism affects Howe's construction.

The construction of $\kappa_{i}$ begins with an explanation of how to transport the discussion in the previous section to the setting which is relevant to tame supercuspidal representations. Let $\operatorname{det}_{i}$ and $\operatorname{tr}_{i}$ denote the determinant and trace maps on $\mathfrak{g}_{i}=$ $\mathfrak{g l}\left(n_{i}, E_{i}\right)$. Define a multiplicative inner product on $\mathfrak{g}$ by $\langle x, y\rangle=\psi_{F}\left(\operatorname{tr}_{0}(x y)\right)$. The orthogonal complement of a subset $S$ of $\mathfrak{g}$ is defined by:

$$
S^{\perp}=\{x \in \mathfrak{g}:\langle x, y\rangle=1 \text {, for all } y \in S\} .
$$

If $S$ is a closed subgroup of $g$ then $\left(S^{\perp}\right)^{\perp}=S$ and if $S_{1}, S_{2}$ are closed subgroups then $\left(S_{1} \cap S_{2}\right)^{\perp}=S_{1}^{\perp}+S_{2}^{\perp}$. When $g \in G$ and $S$ is a subset of $g$ then we have $\left(g S g^{-1}\right)^{\perp}$ $=g S^{\perp} g^{-1}$.

We now state a lemma which describes the orthogonal complements of most importance in the rest of the paper. In order to do this, however, a few more notations are needed. Let $\sigma_{\eta}$ be the anti-involution of $g$ defined by $\sigma_{\eta}(x)=\eta x^{*} \eta^{-1}$. Then the Lie algebra of the unitary group $H$ is 
$\mathfrak{h}=\left\{x \in \mathfrak{g}: \sigma_{\eta}(x)=-x\right\}$.

Now fix an element $c_{i} \in E^{\prime}{ }_{i}$ such that $\theta_{i}^{\prime}(1+x)=\psi_{E_{i}^{\prime}}\left(c_{i} x\right)$, for all $x \in \mathfrak{p}_{i}^{\mu_{i}^{\prime}}$, where $\mu_{i}^{\prime}=\left\lfloor\left(f\left(\theta_{i}^{\prime}\right)+1\right) / 2\right\rfloor$ and $f\left(\theta_{i}^{\prime}\right)$ is the conductoral exponent of $\theta_{i}^{\prime}$. Such an element $c_{i}$ exists and, though it is not unique, the $\operatorname{coset} c_{i}+\mathfrak{p}_{i}^{1-\mu_{i}^{\prime}}$ is well defined. We note that $c_{i}$ must lie in $\mathfrak{p}_{i}^{1-f\left(\theta_{i}^{\prime}\right)}-\mathfrak{p}_{i}^{2-f\left(\theta_{i}^{\prime}\right)}$ and one may easily check that if $\mu_{i}=\left\lfloor\left(f\left(\theta_{i}\right)+1\right) / 2\right\rfloor$, then $\theta_{i}(1+x)=\left\langle c_{i}, x\right\rangle$, for all $x \in \mathfrak{p}_{i}^{\mu_{i}}$.

\section{LEMMA 8.1.}

(i) $\mathfrak{h}^{\perp}=\left\{x \in \mathfrak{g}: \sigma_{\eta}(x)=x\right\}$.

(ii) $E^{\prime}=E \cap \mathfrak{h}^{\perp}$.

(iii) $\mathfrak{b}_{k}^{\perp}=\mathfrak{b}_{1-k}$, for all integers $k$.

(iv) $c_{i} \in\left(\mathfrak{b}_{f_{i}}+\mathfrak{h}\right)^{\perp} \cap E$, for all $i \in\{1, \ldots, r\}$ such that $f_{i}>1$.

(v) If $f_{r}=1$ then $c_{i} \in\left(\mathfrak{b}_{f_{i}}+g \mathfrak{h} g^{-1}\right)^{\perp} \cap E_{r-1}$, for all $i \in\{1, \ldots, r-1\}$ and $g \in G_{r-1}$.

Proof. We first observe that $\mathfrak{h}^{\perp}$ consists of those $x \in \mathfrak{g}$ such that $\left\langle x, y-\sigma_{\eta}(y)\right\rangle=1$, for all $y \in \mathfrak{g}$. This condition can be rewritten as $\langle x, y\rangle=\left\langle x, \sigma_{\eta}(y)\right\rangle=\left\langle\sigma_{\eta}(x), y\right\rangle$ or as $\left\langle x-\sigma_{\eta}(x), y\right\rangle=1$. In the latter form, assertion (i) becomes obvious. Assertion (ii) follows from (i) and the fact that $\sigma_{\eta}(x)=\sigma(x)$, for all $x \in E$. Assertion (ii) is standard. (See [15] or [24], for example.) To prove (iv), we note that from (ii) we have $c_{i} \in E^{\prime}=E \cap \mathfrak{h}^{\perp}$. On the other hand, $c_{i} \in \mathfrak{p}_{i}^{1-f\left(\theta_{i}^{\prime}\right)} \subset \mathfrak{P}_{i}^{1-f\left(\theta_{i}\right)} \subset \mathfrak{P}_{E}^{1-f_{i}} \subset \mathfrak{b}_{1-f_{i}}$. Hence, we have $c_{i} \in \mathfrak{b}_{1-f_{i}} \cap E \cap \mathfrak{h}^{\perp}=\left(\mathfrak{b}_{f_{i}}+\mathfrak{h}\right)^{\perp} \cap E$. The proof of (v) is similar to the proof of (iv), once we observe that $c_{i} \in E_{r-1} \cap g \mathfrak{h}^{\perp} g^{-1}$. The latter fact holds since $g$ commutes with all elements of $E_{r-1}$ and, in particular, with $c_{i}$.

Define the groups

$$
\begin{aligned}
& J_{i}=1+\left(\mathfrak{b}_{\ell_{i}, i-1} \cap \mathfrak{g}_{i}^{\perp}\right)+\mathfrak{b}_{2 \ell_{i}, i}, \\
& J_{i}^{\prime}=1+\left(\mathfrak{b}_{m_{i}, i-1} \cap \mathfrak{g}_{i}^{\perp}\right)+\mathfrak{b}_{2 \ell_{i}, i} .
\end{aligned}
$$

Note that when $f_{i}$ is even, the groups $J_{i}$ and $J_{i}^{\prime}$ are identical since $\ell_{i}=m_{i}$. Define a character $\omega_{i}$ of $E^{\times} B_{0, i} J_{i}^{\prime}$ by $\omega_{i}=\theta_{i} \circ \operatorname{det}_{i}$ on $E^{\times} B_{0, i}$ and $\omega_{i}(x)=\left\langle c_{i}, x-1\right\rangle$ on $J_{i}^{\prime}$. Let $J_{i}^{\prime \prime}=\operatorname{ker}\left(\omega_{i} \mid J_{i}^{\prime}\right)$.

Let $\mathcal{H}_{i}=J_{i} / J_{i}^{\prime \prime}, \mathcal{Z}_{i}=J_{i}^{\prime} / J_{i}^{\prime \prime}$ and $W_{i}=J_{i} / J_{i}^{\prime}$ and define a (multiplicative) symplectic form on $W_{i}$ by

$$
\langle\langle x, y\rangle\rangle_{i}=\omega_{i}\left(x y x^{-1} y^{-1}\right)=\left\langle c_{i},[x-1, y-1]\right\rangle .
$$

(Via the map $1+x \mapsto x$, we obtain an isomorphism of $W_{i}$ with the additive $\mathbb{F}_{p}$-vector space $\left(\mathfrak{b}_{\ell_{i}, i-1} \cap \mathfrak{g}_{i}^{\perp}\right) /\left(\mathfrak{b}_{m_{i}, i-1} \cap \mathfrak{g}_{i}^{\perp}\right)$. It is frequently more convenient to deal with this additive model for $W_{i}$.)

The group $\mathcal{H}_{i}$ turns out to be isomorphic to a Heisenberg group over $\mathbb{F}_{p}$ associated to the symplectic space, however, when defining the Weil representation it is important to be specific about this isomorphism. This is done as follows. If $\mu_{p}$ is the group of complex $p$ th roots of unity then the restriction $\zeta_{i}$ of $\omega_{i}$ to $J_{i}^{\prime}$ gives rise to an exact sequence 


$$
1 \rightarrow J_{i}^{\prime \prime} \rightarrow J_{i}^{\prime} \rightarrow \mu_{p} \rightarrow 1
$$

and an identification of $\mathcal{Z}_{i}$ with $\mu_{p}$. Define $\mathcal{H}_{i}^{\circ}$ to be the standard multiplicative Heisenberg group associated to the symplectic space $W_{i}$, that is, $\mathcal{H}_{i}^{\circ}=W_{i} \ltimes \mu_{p}$ with multiplication defined by

$$
(v, a)(w, b)=\left(v w, a b\langle\langle v, w\rangle\rangle_{i}^{(p+1) / 2}\right) .
$$

Note that $t \mapsto t^{(p+1) / 2}$ should be regarded as the analogue in $\mu_{p}$ of the map $t \mapsto t / 2$ in $F_{p}$.

DEFINITION. Given $i$, a special isomorphism is a homomorphism $v$ : $\mathcal{H}_{i} \rightarrow \mathcal{H}_{i}^{\circ}$ such that the following diagram commutes:

$$
\begin{array}{cccccccc}
1 & \rightarrow \mathcal{Z}_{i} & \rightarrow & \mathcal{H}_{i} & \rightarrow & W_{i} & \rightarrow & 1 \\
\downarrow & \downarrow & & \downarrow & & \downarrow & & \downarrow \\
1 & \rightarrow \mu_{p} & \rightarrow & \mathcal{H}_{i}^{\circ} & \rightarrow & W_{i} & \rightarrow & 1,
\end{array}
$$

where all of the maps other that $v$ are the obvious ones.

This notion is discussed in detail in [31]. We will recall below how, once one specifies a special isomorphism, one obtains a correspondence between admissible quasicharacters and tame supercuspidal representations. In particular, there is a canonical special isomorphism (described in great generality in Proposition 11.4 of [31]) which gives rise to Howe's correspondence, as described in [15] and [24]. Even though the main results of this paper are independent of the choice of special isomorphism, it is necessary to make a specific choice in order to carry out our calculations.

We now define a particularly convenient special isomorphism. This will depend on first defining a polarization of $W_{i}$ which is compatible with with the anti-involution $\sigma_{\eta}$. Define the subgroups of $J_{i}$ by

$$
\begin{aligned}
& J_{i}^{+}=1+\left(\mathfrak{b}_{\ell_{i}, i-1} \cap \mathfrak{g}_{i}^{\perp} \cap \mathfrak{h}^{\perp}\right)+\mathfrak{b}_{2 \ell_{i}, i-1}, \\
& J_{i}^{-}=1+\left(\mathfrak{b}_{\ell_{i}, i-1} \cap \mathfrak{g}_{i}^{\perp} \cap \mathfrak{h}\right)+\mathfrak{b}_{2 \ell_{i}, i-1}
\end{aligned}
$$

and let $\mathcal{H}_{i}^{+}$and $\mathcal{H}_{i}^{-}$, respectively, be the images of $J_{i}^{+}$and $J_{i}^{-}$in $\mathcal{H}_{i}$. These subgroups of $\mathcal{H}_{i}$ satisfy $\mathcal{H}_{i}^{+} \cap \mathcal{Z}_{i}=\{1\}=\mathcal{H}_{i}^{-} \cap \mathcal{Z}_{i}$. Therefore, the natural map $\mathcal{H}_{i} \rightarrow W_{i}$ restricts to give isomorphisms $\mathcal{H}_{i}^{+} \cong W_{i}^{+}$and $\mathcal{H}_{i}^{-} \cong W_{i}^{-}$, where $W_{i}^{+}$and $W_{i}^{-}$are the images of $J_{i}^{+}$and $J_{i}^{-}$, respectively, in $W_{i}$.

LEMMA 8.2. The subspaces $W_{i}^{+}$and $W_{i}^{-}$form a polarization of $W_{i}$.

Proof. We need to show that $W_{i}^{+}$and $W_{i}^{-}$are totally isotropic and $W_{i}=W_{i}^{+} \oplus W_{i}^{-}$. The fact that $W_{i}^{+}$is totally isotropic results from the following observation. If $a, b \in \mathfrak{h}^{\perp}$ then, since $c_{i} \in E^{\prime}=E \cap \mathfrak{h}^{\perp}$ and $[a, b] \in \mathfrak{h}$, we have $\left\langle c_{i},[a, b]\right\rangle=1$. Similarly, one sees that $W_{i}^{-}$is totally isotropic. Next, we note that every $x \in \mathfrak{g}$ has a canonical decomposition $x=x^{+}+x^{-}$, where $x^{+}=\left(x+\sigma_{\eta}(x)\right) / 2$ $\in \mathfrak{h}^{\perp}$ and $x^{-}=\left(x-\sigma_{\eta}(x)\right) / 2 \in \mathfrak{h}$. If $x$ lies in $\mathfrak{b}_{\ell_{i}, i-1}$ then so do its components $x^{+}$and $x^{-}$. It follows that $W_{i}=W_{i}^{+} \oplus W_{i}^{-}$. 
Suppose $h^{+} \in \mathcal{H}_{i}^{+}$and $h^{-} \in \mathcal{H}_{i}^{-}$. Let $w^{+}$and $w^{-}$be the images of $h^{+}$and $h^{-}$in $W^{+}$ and $W^{-}$, respectively. If $z \in \mathcal{Z}_{i}$, we take

$$
v_{i}^{\natural}\left(h^{+} h^{-} z\right)=\left(w^{+} w^{-}, \zeta_{i}(z)\left\langle\left\langle h^{+}, h^{-}\right\rangle\right\rangle^{(p+1) / 2}\right) .
$$

This defines the special isomorphism which we refer to as the natural special isomorphism.

Fix an arbitrary special isomorphism $v$. We now are ready to construct the representation $\kappa_{i}=\kappa_{i}^{v}$ of the group $R=E^{\times} B(\theta)$. Note that $R=R_{i} J_{i} R_{i}^{\prime}$, where $R_{i}=R \cap G_{i}=E^{\times} B\left(\theta_{r}\right) \ldots B\left(\theta_{i+1}\right)$ and $R_{i}^{\prime}=B\left(\theta_{i-1}\right) \ldots B\left(\theta_{1}\right)$. The symplectic group $\mathcal{S}_{i}=\operatorname{Sp}\left(W_{i}\right)$ acts on $\mathcal{H}_{i}^{\circ}$ by $s \cdot(v, a)=(s \cdot v, a)$. The main significance of choosing a special isomorphism is that it is used to transfer the action of $\mathcal{S}_{i}$ on $\mathcal{H}_{i}^{\circ}$ to an action of $\mathcal{S}_{i}$ on $\mathcal{H}_{i}$. Specifically, $\mathcal{S}_{i}$ acts on $\mathcal{H}_{i}$ by $s \cdot h=v^{-1}(s \cdot v(h))$. Let $\left(\rho_{i}, V_{i}\right)$ be the induced representation $\operatorname{Ind}_{\mathcal{H}_{i}^{+} \mathcal{Z}_{i}}\left(\zeta_{i}^{+}\right)$, where $\zeta_{i}^{+}$is the character of $\mathcal{H}_{i}^{+} \mathcal{Z}_{i}$ defined by $\zeta_{i}^{+}\left(h^{+} z\right)=\zeta_{i}(z)$ when $h^{+} \in \mathcal{H}_{i}^{+}$and $z \in \mathcal{Z}_{i}$. This is a Heisenberg representation. According to Proposition 7.2, $\operatorname{Hom}_{\mathcal{H}_{i}^{-}}\left(\rho_{i}, 1\right)$ has dimension one and is generated by the linear form $\lambda_{i}(\varphi)=\varphi(1)$. As discussed above, the representation $\rho_{i}$ extends uniquely to a Weil representation $\left(\hat{\rho}_{i}, V_{i}\right)$ of $\mathcal{S}_{i} \times \mathcal{H}_{i}$. We stress that the definition of $\mathcal{S}_{i} \ltimes \mathcal{H}_{i}$ and the definition of the Weil representation on $\mathcal{S}_{i}$ both depend on the choice of special isomorphism. It should also be emphasized that the discussion above becomes trivial in the case that $f_{i}$ is even, since then $W_{i}=0$.

Let $\operatorname{Sp}\left(\mathcal{H}_{i}\right)$ be the group of all automorphisms of $\mathcal{H}_{i}$ which (modulo $\mathcal{Z}_{i}$ ) define elements of $\mathcal{S}_{i}$ and define the group $\operatorname{Sp}\left(\mathcal{H}_{i}^{\circ}\right)$ similarly. Then the natural maps $\operatorname{Sp}\left(\mathcal{H}_{i}\right) \rightarrow \mathcal{S}_{i}$ and $\operatorname{Sp}\left(\mathcal{H}_{i}^{\circ}\right) \rightarrow \mathcal{S}_{i}$ are isomorphisms. The action of the group $E^{\times} B_{0, i}$ by conjugation on $\mathcal{H}_{i}$ defines a homomorphism $\xi_{i}$ : $E^{\times} B_{0, i} \rightarrow \operatorname{Sp}\left(\mathcal{H}_{i}\right)$ with the property that, for all $g \in E^{\times} B_{0, i}$, the composite map $v \circ \xi_{i}(g) \circ v^{-1}$ is an element of $\operatorname{Sp}\left(\mathcal{H}_{i}^{\circ}\right)$.

The homomorphism $\xi_{i}$ extends to a homomorphism $E^{\times} B_{0, i} \times \mathcal{H}_{i} \rightarrow \mathcal{S}_{i} \times \mathcal{H}_{i}$ which we use to pull back the Weil representation $\hat{\rho}_{i}$ to a representation $\kappa_{i}^{\circ}$ of $E^{\times} B_{0, i} \ltimes \mathcal{H}_{i}$ on the space $V_{i}$. We also have another representation of $E^{\times} B_{0, i} \ltimes \mathcal{H}_{i}$ obtained by inflating $\omega_{i}$ up from $E^{\times} B_{0, i}$. This representation will be denoted $\inf \left(\omega_{i}\right)$. Note that whenever $x \in B_{2 \ell_{i}, i}=E^{\times} B_{0, i} \cap J_{i}$ the representation $\kappa_{i}^{\circ} \otimes \inf \left(\omega_{i}\right)$ is trivial on the element $\left(x, x^{-1}\right) \in E^{\times} B_{0, i} \ltimes \mathcal{H}_{i}$. Therefore, $\kappa_{i}^{\circ} \otimes \inf \left(\omega_{i}\right)$ factors through $E^{\times} B_{0, i} J_{i}=$ $E^{\times} B_{0, i} B\left(\theta_{i}\right)$. The restriction of the resulting representation of $E^{\times} B_{0, i} B\left(\theta_{i}\right)$ to $R \cap G_{i-1}=E^{\times} B\left(\theta_{r}\right) \ldots B\left(\theta_{i}\right)$ defines our desired representation $\kappa_{i}$ on $R \cap G_{i-1}$. If $i=1$ this defines $\kappa_{i}$ on $R$. Otherwise, if $i>1$, we take $\kappa_{i}(1+x)=$ $\left\langle c_{i}, x\right\rangle$ on $B\left(\theta_{i-1}\right) \ldots B\left(\theta_{1}\right)$ to complete the definition of $\kappa_{i}$ on $R$ in the case for which $f_{i}>1$.

Since the construction of $\kappa_{i}$ when $f_{i}>1$ is somewhat complicated, let us now summarize its main features. First of all, the representation space of $\kappa_{i}$ is the same as the representation space $V_{i}$ of the Heisenberg representation $\rho_{i}$. Secondly, if $f_{i}$ is even then $\kappa_{i}$ is simply the one-dimensional representation of $R$ defined on $E^{\times} B\left(\theta_{r}\right) \cdots B\left(\theta_{i}\right)$ by restricting $\omega_{i}$ and on $B\left(\theta_{i-1}\right) \ldots B\left(\theta_{1}\right)$ by $\kappa_{i}(1+x)=\left\langle c_{i}, x\right\rangle$. Finally, if $f_{i}$ is odd and greater than 1 , then 


$$
\kappa_{i}(x y z)=\theta_{i}\left(\operatorname{det}_{i}(x)\right) \hat{\rho}_{i}\left(\xi_{i}(x)\right) \rho_{i}(y)\left\langle c_{i}, z-1\right\rangle,
$$

where $x \in R_{i}, y \in J_{i}$ and $z \in R_{i}^{\prime}$. Since the construction of $\kappa_{i}$ depends on the choice of the special isomorphism $v$, it is more appropriate to use notations such $\kappa_{i}^{v}, \hat{\rho}_{i}^{v}$ and $\mathcal{S}_{i} \ltimes{ }_{v} \mathcal{H}_{i}$, rather than $\kappa_{i}, \hat{\rho}_{i}$ and $\mathcal{S}_{i} \ltimes \mathcal{H}_{i}$, to reflect this dependence. We will use these more complete notations when it is necessary to signify the choice of special isomorphism. When using the natural special isomorphism, we use notations such as $\kappa_{i}^{\natural}, \hat{\rho}_{i}^{\natural}$ and $\mathcal{S}_{i} \ltimes_{\natural} \mathcal{H}_{i}$.

\section{Varying the Special Isomorphism}

The notations in this section are the same as in the previous section. Recall that we have defined an anti-involution of $\mathfrak{g}$ and, by restriction, of $G$ by the formula $\sigma_{\eta}(x)=\eta x^{*} \eta^{-1}$, where the Hermitian matrix $\eta$ is always chosen to lie in $E^{\times}$. Let $\imath_{\eta}$ be the involution of $G$ given by $\imath_{\eta}(g)=\sigma_{\eta}(g)^{-1}$.

Let $\tilde{\eta} \in k_{r}^{\prime}$ be the image in $k_{r}^{\prime}$ of $\varpi_{r}^{\prime k} \eta$, where $k$ is the integer for which $\varpi_{r}^{\prime k} \eta \in \mathcal{O}_{r}^{\prime x}$. When $E^{\prime} / E_{r-1}^{\prime}$ is unramified, we will assume that $\varpi_{r}^{\prime}=\varpi_{r-1}^{\prime}$. Let

$$
\mathcal{G}=(R \cap B) /\left(R \cap B_{1}\right) \cong \begin{cases}k_{r}^{\times}, & \text {if } f_{r}>1, \\ \operatorname{GL}\left(n_{r-1}, k_{r-1}\right), & \text { if } f_{r}=1,\end{cases}
$$

where $n_{i}=\left[E: E_{i}\right]$. Let

$$
\mathcal{B}=(R \cap H) /\left(R \cap H \cap B_{1}\right) .
$$

Note that $R \cap H=R \cap H \cap B$ and, therefore, $\mathcal{B}$ may be viewed as a subgroup of $\mathcal{G}$. We have

$$
\mathcal{B} \cong \begin{cases}\mathrm{U}\left(1, k_{r} / k_{r}^{\prime}\right), & \text { if } f_{r}>1 \text { and } E / E^{\prime} \text { is unramified, } \\ \{ \pm 1\}, & \text { if } f_{r}>1 \text { and } E / E^{\prime} \text { is ramified, } \\ \mathrm{U}\left(\tilde{\eta}, k_{r-1} / k_{r-1}^{\prime}\right), & \text { if } f_{r}=1 \text { and } E / E^{\prime} \text { is unramified, } \\ \mathrm{O}\left(\tilde{\eta}, k_{r-1}\right), & \text { if } f_{r}=1 \text { and } E / E^{\prime} \text { is ramified. }\end{cases}
$$

Here, if $L / K$ is quadratic extension with respect to which $x$ is an invertible hermitian matrix of rank $s$, then $\mathrm{U}(x, L / K)$ denotes the unitary group in $\operatorname{GL}(s, L)$ defined by $g x g^{*}=x$. The notation $\mathrm{O}(x, L)$ for orthogonal groups is similar.

LEMMA 9.1. Suppose $\kappa_{i}=\kappa_{i}^{v}$, for some special isomorphism $v: \mathcal{H}_{i} \rightarrow \mathcal{H}_{i}^{\circ}$. Then the contragredient representation $\tilde{\kappa}_{i}$ is equivalent to $\kappa_{i} \circ l_{\eta}$.

Proof. Throughout the proof, we assume we have fixed the special isomorphism $v$ and we suppress it from the notation. The central character $\zeta_{i}$ of the Heisenberg representation $\rho_{i}$ is given by restricting the character $\omega_{i}$ to $J_{i}^{\prime}$. A straightforward computation shows that $\zeta_{i} \circ \sigma_{\eta}=\zeta_{i}$ or, equivalently, $\zeta_{i} \circ l_{\eta}=\zeta_{i}^{-1}$. Up to isomorphism, the contragredient representation $\tilde{\rho}_{i}$ is the Heisenberg representation of $\mathcal{H}_{i}$ with central character $\zeta_{i}^{-1}$. Therefore, $\tilde{\rho}_{i}$ is equivalent to $\rho_{i} \circ l_{\eta}$. One of the basic properties of the Weil representation $\hat{\rho}_{i}$ (see [6]) is that its contragredient is the Weil representation which is the unique extension of $\tilde{\rho}_{i}$ to $\mathcal{S}_{i} \ltimes \mathcal{H}_{i}$. Hence, 
the contragredient of $\hat{\rho}_{i}$ must be equivalent to $\hat{\rho}_{i} \circ l_{\eta}$. Therefore, $\tilde{\kappa}_{i}^{\circ} \otimes \inf \left(\omega_{i}\right)^{\sim}$ is equivalent to $\left(\kappa_{i}^{\circ} \otimes \inf \left(\omega_{i}\right)\right) \circ \sigma_{\eta}$ and our assertion follows.

LEMMA 9.2. Given any two special isomorphisms $v_{1}$ and $v_{2}$, there exists a character $\chi$ of $R$ such that $\kappa_{i}^{\nu_{2}}=\kappa_{i}^{\nu_{1}} \otimes \chi$ and $\chi \circ l_{\eta}=\chi^{-1}$. Furthermore:

(i) If $f_{r}=1, E / E^{\prime}$ is ramified and $n_{r-1}$ is even then $\chi^{2} \mid R \cap H \equiv 1$.

(ii) In all other cases, $\chi \mid R \cap H \equiv 1$.

Proof. By definition, $\kappa_{i}^{\nu_{1}}$ and $\kappa_{i}^{v_{2}}$ coincide on $R \cap B_{1}$. Therefore, there exists a character $\chi$ of $R$ which is trivial on $R \cap B_{1}$ and satisfies $\kappa_{i}^{v_{2}}=\kappa_{i}^{\nu_{1}} \otimes \chi$. By Lemma 9.1, $\chi \circ l_{\eta}=\chi^{-1}$.

Suppose $f_{r}>1$. Then $R /\left(R \cap B_{1}\right) \cong E /\left(1+\mathfrak{B}_{E}\right)$. For each $\alpha \in E^{\times}$, we have $\chi(\sigma(\alpha))=\chi\left(l_{\eta}(\alpha)\right)^{-1}=\chi(\alpha)$. Therefore, since $E^{\times} \cap H$ is the unitary group $\mathrm{U}\left(1, E / E^{\prime}\right)$, Hilbert's Theorem 90 implies $\chi \mid E^{\times} \cap H \equiv 1$.

Now suppose that $f_{r}=1$. There exists a character $\chi^{\prime}$ of $\mathfrak{D}_{r-1}^{\times}$which is trivial on $1+\mathfrak{B}_{r-1}$ and is such that $\chi$ is the inflation of $\chi^{\prime} \circ \operatorname{det}_{r-1}$ from $B_{0, r-1}$ to $R \cap B=B_{0, r-1}\left(R \cap B_{1}\right)$. The condition $\chi \circ l_{\eta}=\chi^{-1}$ implies $\chi^{\prime} \circ \sigma=\chi^{\prime}$. Note that $\operatorname{det}_{r-1}(x)=\sigma\left(\operatorname{det}_{r-1}(x)\right)^{-1}$, for all $x \in B_{0, r-1} \cap H$. If $E / E^{\prime}$ is unramified, we apply Hilbert's Theorem 90 to $k_{r-1} / k_{r-1}^{\prime}$ to deduce that $\chi \mid B_{0, r-1} \cap H \equiv 1$. Now suppose $E / E^{\prime}$ is ramified. Then $\chi^{\prime}\left(\operatorname{det}_{r-1}(x)\right)=\chi^{\prime}\left(\operatorname{det}_{r-1}(x)\right)^{-1}$, for all $x \in B_{0, r-1} \cap H$, since $\chi^{\prime}$ is trivial on $1+\mathfrak{B}_{r-1}$ and $\sigma$ induces the identity map on $k_{r-1}$. In other words, we have $\chi^{\prime 2} \mid B_{0, r-1} \cap H \equiv 1$. Since we may choose $\varpi_{r}$ so that $\sigma\left(\varpi_{r}\right)=-\varpi_{r}$, it follows that $\chi(-1)=1$. Thus $\chi^{\prime}\left((-1)^{n_{r-1}}\right)=1$. If $n_{r-1}$ is odd this implies $\chi^{\prime}(-1)=1$, which completes the proof.

Recall that $\mathcal{P}_{i}$ is the stabilizer of $W_{i}^{+}$in $\mathcal{S}_{i}$. The subgroup $\mathcal{M}_{i}$ of elements of $\mathcal{P}_{i}$ which also stabilize $W_{i}^{-}$forms a Levi component of $\mathcal{P}_{i}$. Suppose $x \in R_{i} \cap H$. Then, since $x \in E^{\times} B_{0, i} \cap H=B_{0, i} \cap H$, the operator $\operatorname{Ad}(x)$ stabilizes $\mathfrak{b}_{\ell_{i}, i-1} \cap \mathfrak{g}_{i}^{\perp} \cap \mathfrak{h}^{\perp}$ and $\mathfrak{b}_{\ell_{i}, i-1} \cap \mathfrak{g}_{i}^{\perp} \cap \mathfrak{h}$ and, hence, $\xi_{i}(x) \in \mathcal{M}_{i}$. We may choose an $\mathbb{F}_{p}$-basis of $W_{i}^{+}$which gives rise to an identification $W_{i}^{+} \cong \mathbb{F}_{p}^{\ell}$, where $p^{2 \ell+1}$ is the order of $\mathcal{H}_{i}$. We also obtain an identification $\mathcal{M}_{i} \cong \operatorname{GL}\left(\ell, \mathbb{F}_{p}\right)$. There is a unique character $\mu_{i}$ of $\mathcal{M}_{i}$ of order two. It corresponds to the character $g \mapsto(\operatorname{det} g)^{(p-1) / 2}$ of $\operatorname{GL}\left(\ell, \mathbb{F}_{p}\right)$ under any isomorphism of $\mathcal{M}_{i}$ with $\operatorname{GL}\left(\ell, \mathbb{F}_{p}\right)$. The following result involves the Weil representation constructed using the natural special isomorphism:

LEMMA 9.3. If $f_{i}>1, \varphi \in V_{i}, x \in R_{i} \cap H$ and $h \in \mathcal{H}_{i}$, then

$$
\hat{\rho}_{i}^{\natural}\left(\xi_{i}(x)\right) \varphi(h)=\mu_{i}\left(\xi_{i}(x)\right) \varphi\left(\xi_{i}(x)^{-1} \cdot h\right),
$$

where the action of $\mathcal{S}_{i}$ on $\mathcal{H}_{i}$ in the expression $\xi_{i}(x)^{-1} \cdot h$ is defined relative to the natural special isomorphism. Furthermore, $\mu_{i}\left(\xi_{i}(x)\right)=1$, unless $f_{r}=1, E / E^{\prime}$ is ramified and $n_{r-1}$ is even. 
Proof. Choose $\varphi, x$ and $h$ as in the hypothesis of the lemma. We will assume $f_{i}$ is odd, since otherwise our claim is trivial. The stated formula for $\hat{\rho}_{i}^{\natural}\left(\xi_{i}(x)\right) \varphi(h)$ follows directly from the discussion in $\S 2$ of [6]. (We should warn the reader that in [6] the space $V_{i}$ is replaced by the space of all functions on $W_{i}^{-}$. The identification of $V_{i}$ with the latter space is simply given by restricting functions on $\mathcal{H}_{i}$ to functions on $W_{i}^{-}$.)

It remains to compute $\mu_{i}\left(\xi_{i}(x)\right)$. We first observe that $\xi_{i} \mid B_{1, i} \equiv 1$ and thus the restriction of $\xi_{i}$ to $R_{i} \cap H$ factors through the quotient group

$$
\mathcal{B}=(R \cap H) /\left(R \cap H \cap B_{1}\right)=\left(R_{i} \cap H\right) /\left(R_{i} \cap H \cap B_{1}\right) .
$$

Moreover, if $x \in R_{i} \cap H$ then, viewing $\xi_{i}(x)$ as an element of $\mathcal{S}_{i}$, we note that $\xi_{i}(x)\left(W_{i}^{+}\right) \subset W_{i}^{+}$and $\xi_{i}(x)\left(W_{i}^{-}\right) \subset W_{i}^{-}$. Therefore, $\xi$ gives rise to a homomorphism $\xi_{i}^{\prime}: \mathcal{B} \rightarrow \mathcal{M}_{i}$. We must show that the quadratic character $\mu_{i} \circ \xi_{i}^{\prime}$ of $\mathcal{B}$ is trivial except in the case in which $f_{r}=1, E / E^{\prime}$ is ramified and $n_{r-1}$ is even.

If $f_{i}$ is even or if $E / E^{\prime}$ is ramified and $f_{r}>1$ then our assertion follows from the fact that $\xi_{i}^{\prime}$ itself is trivial. Another case which may be easily excluded is the case in which $E / E^{\prime}$ is ramified and $n_{r-1}$ is odd. In this situation, we first observe that $\mu_{i} \circ \xi_{i}^{\prime}$ is a quadratic character of an orthogonal group and, moreover, every quadratic character of the orthogonal group is trivial on the special orthogonal group. Furthermore, $\mu_{i}\left(\xi^{\prime}(-1)\right)=1$, since $\xi^{\prime}(-1)=1$, and our claim follows.

It remains to treat the case in which $E / E^{\prime}$ is unramified. We assume $\varpi_{r}=\varpi_{r}^{\prime}$ and, in addition, if $f_{r}=1$, we assume $\varpi_{r}=\varpi_{r-1}=\varpi_{r}^{\prime}=\varpi_{r-1}^{\prime}$. Let $\eta^{\prime}=\varpi_{r}^{-\ell_{i}} \eta$. Since $\eta^{\prime}$ lies in $E^{\prime \times}$, it is an invertible Hermitian matrix and there is an associated unitary group $H_{\eta^{\prime}}$ with Lie algebra $\mathfrak{h}_{\eta^{\prime}}$. If $\sigma_{\eta^{\prime}}$ is the anti-involution of $\mathfrak{g}$ given by $\sigma_{\eta^{\prime}}(x)=\eta^{\prime} x^{*} \eta^{\prime}-1$ then the elements of $H_{\eta^{\prime}}$ satisfy $\sigma_{\eta}(h)=h^{-1}$ and the elements of $\mathfrak{h}_{\eta^{\prime}}$ satisfy $\sigma_{\eta^{\prime}}(x)=-x$. There is a finite Lie group $L$ with Lie algebra $\mathfrak{l}$ defined by

$$
\begin{aligned}
& L=\left(B_{0, i-1} \cap H_{\eta^{\prime}}\right) /\left(B_{1, i-1} \cap H_{\eta^{\prime}}\right), \\
& \mathfrak{l}=\left(\mathfrak{b}_{0, i-1} \cap \mathfrak{h}_{\eta^{\prime}}\right) /\left(\mathfrak{b}_{1, i-1} \cap \mathfrak{h}_{\eta^{\prime}}\right) .
\end{aligned}
$$

The structure of $L$ is partially described as follows. Note first that $B_{0, i-1} / B_{1, i-1}$ is isomorphic to a product of $e\left(E / E_{i-1}\right)$ copies of $\operatorname{GL}\left(f\left(E / E_{i-1}\right), k_{i-1}\right)$. There is an automorphism of order two defined on this group by $\imath_{\eta}(g)=\sigma_{\eta}(g)^{-1}$ whose group of fixed points is $L$. A given factor in the product decomposition of $B_{0, i-1} / B_{1, i-1}$ is either paired with another factor by $\imath_{\eta}$ or it is preserved by $\imath_{\eta}$. The group of $\imath_{\eta}$-fixed points for each set of paired factors is isomorphic to $\operatorname{GL}\left(f\left(E / E_{i-1}\right), k_{i-1}\right)$, while the group of $l_{\eta}$-fixed points for each stable factor is isomorphic to $\mathrm{U}\left(f\left(E / E_{i-1}\right), k_{i-1} / k_{i-1}^{\prime}\right)$. Therefore, $L$ is isomorphic to a product of general linear groups and unitary groups. What is most relevant for us is that $L$ is the $\mathbb{F}_{p}$-rational points of a connected reductive group defined over $\mathbb{F}_{p}$ and $\mathfrak{l}$ is its Lie algebra over $\mathbb{F}_{p}$. It is known that in such a situation if $g \in L$ then the determinant of $\operatorname{Ad}(g): \mathfrak{l} \rightarrow \mathfrak{l}$ is always 1 . 
Now define

$$
L^{\prime}=B_{0, r-1} \cap H, \quad \text { if } f_{r}=1,
$$

and

$$
L^{\prime}=E^{\times} \cap H=U\left(1, E / E^{\prime}\right), \quad \text { if } f_{r}>1 .
$$

Then $L^{\prime}$ contains a set of coset representatives for $\mathcal{B}$ and $L^{\prime} \subset B_{0, i-1} \cap H_{\eta^{\prime}}$. (To see that $L^{\prime} \subset B_{0, i-1} \cap H_{\eta^{\prime}}$ when $f_{r}=1$, use the fact that $\sigma_{\eta}=\sigma_{\eta^{\prime}}$ on $\mathfrak{g}_{r-1}$, since $\varpi_{r}^{\ell_{i}} \in E^{\prime}{ }_{r-1}$.) It follows that $\mathcal{B}$ may be regarded as a subgroup of $L$.

Next, we observe that, in fact, $\mathfrak{h}_{\eta^{\prime}}=\varpi_{r}^{-\ell_{i}} \mathfrak{h}$ from which it follows that $x \mapsto \varpi_{r}^{\ell_{i}} x$ gives an isomorphism of $\mathfrak{l}$ with $\mathfrak{l}^{\prime}=\left(\mathfrak{b}_{\ell_{i}, i-1} \cap \mathfrak{h}\right) /\left(\mathfrak{b}_{m_{i}, i-1} \cap \mathfrak{h}\right)$. Note that $\mathfrak{l}$ decomposes as $\mathfrak{l}=\mathfrak{l}_{1} \oplus \mathfrak{l}_{2}$, with

$$
\begin{aligned}
& \mathfrak{l}_{1}=\left(\mathfrak{b}_{0, i-1} \cap \mathfrak{g}_{i}^{\perp} \cap \mathfrak{h}_{\eta^{\prime}}\right) /\left(\mathfrak{b}_{1, i-1} \cap \mathfrak{g}_{i}^{\perp} \cap \mathfrak{h}_{\eta^{\prime}}\right), \\
& \mathfrak{l}_{2}=\left(\mathfrak{b}_{0, i} \cap \mathfrak{h}_{\eta^{\prime}}\right) /\left(\mathfrak{b}_{1, i} \cap \mathfrak{h}_{\eta^{\prime}}\right),
\end{aligned}
$$

and there is an analogous decomposition $\mathfrak{l}^{\prime}=\mathfrak{l}_{1}^{\prime} \oplus \mathfrak{l}_{2}^{\prime}$. The summand $\mathfrak{l}_{1}^{\prime}$ is just the image of $W_{i}^{-}$under $1+x \mapsto x$. Let $L_{2}=\left(B_{0, i} \cap H_{\eta^{\prime}}\right) /\left(B_{1, i} \cap H_{\eta^{\prime}}\right)$. Note that $L^{\prime} \subset$ $B_{0, i} \cap H_{\eta^{\prime}}$ and therefore the elements of $L^{\prime}$ project to elements of $L_{2}$, as well as $L$.

Returning to the task at hand, we need to compute $\mu_{i}\left(\xi_{i}(x)\right)$ when $x \in L^{\prime}$. This amounts to determining whether or not the determinant of $\operatorname{Ad}(x)$ on $W_{i}^{+}$is a square. If $x_{+} \in \mathrm{GL}\left(W_{i}^{+}\right)$and $x_{-} \in \mathrm{GL}\left(W_{i}^{-}\right)$are the linear transformations determined by the adjoint action of $x$, then it is easy to see that $\operatorname{det} x_{+}=\operatorname{det} x_{-}^{-1}$. (See [6].) Therefore, it suffices to compute $\operatorname{det} \operatorname{Ad}(x) \mid \mathfrak{I}_{1}^{\prime}$. We have

$$
\operatorname{det} \operatorname{Ad}(x) \mid \mathfrak{l}_{1}^{\prime}=\frac{\operatorname{det} \operatorname{Ad}(x) \mid \mathfrak{l}^{\prime}}{\operatorname{det} \operatorname{Ad}(x) \mid \mathfrak{I}_{2}^{\prime}}=\frac{\operatorname{det} \operatorname{Ad}(x) \mid \mathfrak{l}}{\operatorname{det} \operatorname{Ad}(x) \mid \mathfrak{I}_{2}} .
$$

Here, the second equality follows from the fact that if $\varphi$ is the map from $\mathfrak{l}^{\prime}$ to $\mathfrak{l}$ given by $y \mapsto \varpi_{r}^{\ell_{i}} y$ then $\varphi(\operatorname{Ad}(x) y)=\operatorname{Ad}(x) \varphi(y)$, since $x \in L^{\prime}$. By the argument given above, we know that $\operatorname{det} \operatorname{Ad}(x) \mid \mathfrak{l}=1$. A similar argument also applies to $\mathfrak{I}_{2}$ and allows us to deduce our result.

\section{Invariant Linear Forms on the Space of $\kappa_{i}$ when $f_{i}>1$}

The purpose of this section is to prove the following proposition:

PROPOSITION 10.1. Assume $f_{i}>1$ and $\kappa_{i}=\kappa_{i}^{v}$ for some special isomorphism $v: \mathcal{H}_{i} \rightarrow \mathcal{H}_{i}^{\circ}$. Then there exists a quadratic character $\vartheta_{i}$ of $R \cap H$ such that

$$
\operatorname{Hom}_{R \cap H}\left(\kappa_{i}, \vartheta_{i}\right)=\operatorname{Hom}_{B\left(\theta_{i}\right) \cap H}\left(\kappa_{i}, 1\right)
$$

is the one-dimensional space spanned by the linear form $\lambda_{i}(\varphi)=\varphi(1)$. Moreover, $\vartheta_{i}$ is trivial unless $f_{r}=1, E / E^{\prime}$ is ramified and $n_{r-1}$ is even.

Recall that a character $\chi$ is said to be 'quadratic' if $\chi^{2}=1$. The proof of Proposition 10.1 uses two lemmas: 
LEMMA 10.2. Let $G$ be a group and $H$ the subgroup of fixed points of an automorphism $\alpha$ of order two. Assume that $A$ and $B$ are $\alpha$-stable subgroups of $G$ such that $H^{1}(\{1, \alpha\}, A \cap B)=1$, that is, the sets

$$
Z^{1}=\left\{x \in A \cap B: \alpha(x)=x^{-1}\right\} \quad \text { and } \quad B^{1}=\left\{z \alpha(z)^{-1}: z \in A \cap B\right\}
$$

are identical. Then $A B \cap H=(A \cap H)(B \cap H)$.

Proof. Under the stated assumptions, suppose that $a \in A, b \in B$ and $a b \in H$. Then $a b=\alpha(a) \alpha(b)$ implies $a^{-1} \alpha(a)=b \alpha(b)^{-1} \in Z^{1}$. Therefore, there exists $z \in A \cap B$ such that $a^{-1} \alpha(a)=z \alpha(z)^{-1}$. We now have $a b=(a z)\left(z^{-1} b\right)$, where $a z \in A \cap H$ and $z^{-1} b \in B \cap H$.

Suppose $\epsilon_{i}: \mathfrak{b}_{\ell_{i}, i-1} \rightarrow B\left(\theta_{i}\right)$ is the Cayley transform defined by

$$
\epsilon_{i}(x)=(1+x / 2)(1-x / 2)^{-1} .
$$

This map is bijective with inverse $\epsilon_{i}^{-1}(y)=-4 \epsilon_{i}(-2 y)$. We also observe that $\epsilon_{i}(x) \equiv 1+x$ modulo $\mathfrak{b}_{2 \ell_{i}, i-1}$. The Cayley transform commutes with the involution $\sigma_{\eta}(x)=\eta x^{*} \eta^{-1}$ of $\mathfrak{g}$.

LEMMA 10.3. The Cayley transform $\epsilon_{i}$ restricts to a bijection $\mathfrak{b}_{\ell_{i}, i-1} \cap \mathfrak{h} \cong B\left(\theta_{i}\right) \cap H$.

Proof. Suppose $x \in \mathfrak{b}_{\ell_{i}, i-1} \cap \mathfrak{h}$. Then $\sigma_{\eta}\left(\epsilon_{i}(x)\right)=\epsilon_{i}\left(\sigma_{\eta}(x)\right)=\epsilon_{i}(-x)=\epsilon_{i}(x)^{-1}$. Thus $\epsilon_{i}(x) \in B\left(\theta_{i}\right) \cap H$. Similarly, one shows that if $y \in B\left(\theta_{i}\right)$ then $\epsilon_{i}^{-1}(y) \in \mathfrak{b}_{\ell_{i}, i-1} \cap \mathfrak{h}$.

Proof of Proposition 10.1. Consider first the action of $\kappa_{i}^{\natural}$ on $V_{i}$. As before, we write $R=R_{i} J_{i} R_{i}^{\prime}$ and note that each factor is stable under the automorphism $l_{\eta}(g)=$ $\sigma_{\eta}(g)^{-1}$. Applying Lemma 10.2, we see there is a corresponding decomposition of $R \cap H$. Define a character of $R \cap H$ by $\chi(x y z)=\mu_{i}\left(\xi_{i}(x)\right)$, where $x \in R_{i} \cap H$, $y \in J_{i} \cap H$ and $z \in R_{i}^{\prime} \cap H$. Since, up to scalar multiples, $\lambda_{i}$ is the unique nonzero $\left(J_{i} \cap H\right)$-invariant linear form on $V_{i}$, it suffices to show that $\lambda_{i}$ is invariant under $R_{i}^{\prime} \cap H$ and transforms by $\chi$ on $R_{i} \cap H$ in order to prove our assertions for $\kappa_{i}^{\natural}$.

To prove invariance under $R_{i}^{\prime} \cap H$, it suffices to show invariance under $B\left(\theta_{j}\right) \cap H$ for each $j<i$, according to Lemma 10.2. So let us suppose that $z \in B\left(\theta_{j}\right) \cap H$. It suffices to show that $\left\langle c_{i}, z-1\right\rangle=1$. There must exist $x \in \mathfrak{b}_{\ell_{j}, j-1} \cap \mathfrak{h}$ such that $z=\epsilon_{j}(x)$. Since $z-1 \equiv x$ modulo $\mathfrak{b}_{2 \ell_{j}, j-1}$, we conclude that $z-1$ lies in $\left(\mathfrak{b}_{\ell_{j}, j-1} \cap \mathfrak{h}\right)+\mathfrak{b}_{2 \ell_{j}, j-1} \subset \mathfrak{h}+\mathfrak{b}_{f_{i}}$. Applying Lemma 8.1(iv), it now follows that $\lambda_{i}$ must be invariant under $R_{i}^{\prime} \cap H$.

Next, we consider how $\lambda_{i}$ transforms under $R_{i} \cap H$. According to Lemma 9.3, we have

$$
\lambda_{i}\left(\kappa_{i}^{\natural}(x) \varphi\right)=\lambda_{i}\left(\omega_{i}(x) \hat{\rho}_{i}^{\natural}\left(\xi_{i}(x)\right) \varphi\right)=\omega_{i}(x) \mu_{i}\left(\xi_{i}(x)\right) \varphi(1),
$$

for all $x \in R_{i} \cap H$. Therefore, it suffices to show that $\omega_{i}$ or, equivalently, $\theta_{i} \circ \operatorname{det}_{i}$ is trivial on $R_{i} \cap H$. Recall that $\eta \in E^{\prime \times}$ and thus $\eta \in G_{i}$. Observe also that $R_{i} \cap H$ is contained in the group $H_{i}=G_{i} \cap H$. The image of $H_{i}$ under $\operatorname{det}_{i}$ is contained in $\mathrm{U}\left(1, E_{i} / E_{i}^{\prime}\right)$. Since $\theta_{i}$ is trivial on the latter group, our claims regarding $\kappa_{i}^{\natural}$ follow. 
To deduce what is asserted about $\kappa_{i}^{v}$, for arbitrary $v$, one can now simply apply Lemma 9.2.

\section{Three Reductions of the Main Problem}

The objective of this paper is to study the spaces $\operatorname{Hom}_{H}(\pi, 1)$ associated to the tame supercuspidal representation $\pi=\operatorname{Ind}_{R}^{G}(\kappa)$. Towards this end, we discuss in this section three reductions of the main problem. The first says that we are justified in assuming that $\theta_{0}=1$. The second reduces our problem to the problem of looking at the various spaces $\operatorname{Hom}_{R \cap g \mathrm{Hg}^{-1}}(\kappa, 1)$, as $g$ varies over a set of representatives for the double coset space $R \backslash G / H$. The final reduction says that it suffices to look at the double coset $R H$. We complete the third reduction step in the next section, where we consider the case in which $f_{r}=1$.

Recall that $\kappa=\kappa_{0} \otimes \cdots \otimes \kappa_{r}$, where $\kappa_{0}$ is the character of $R$ given by $\theta_{0} \circ$ det. Let $\kappa^{(0)}=\kappa_{1} \otimes \cdots \otimes \kappa_{r}$.

LEMMA 11.1. $\operatorname{Hom}_{H}\left(\operatorname{Ind}_{R}^{G}(\kappa), 1\right) \cong \operatorname{Hom}_{H}\left(\operatorname{Ind}_{R}^{G}\left(\kappa^{(0)}\right), 1\right)$.

Proof. We have an isomorphism of $\operatorname{Ind}_{R}^{G}\left(\kappa^{(0)}\right) \otimes \theta_{0}$ with $\operatorname{Ind}_{R}^{G}(\kappa)$ given by $f \mapsto \tilde{f}$, where $\tilde{f}(g)=\theta_{0}(\operatorname{det} g) f(g)$. Since $\theta_{0} \circ$ det is trivial on $H$, the identity map gives an isomorphism between $\operatorname{Hom}_{H}\left(\operatorname{Ind}_{R}^{G}\left(\kappa^{(0)}\right) \otimes \theta_{0}, 1\right)$ and $\operatorname{Hom}_{H}\left(\operatorname{Ind}_{R}^{G}\left(\kappa^{(0)}\right), 1\right)$. Our claim follows.

In light of Lemma 11.1, we will assume $\theta_{0}=1$ for the rest of the paper. We now state the second reduction step.

LEMMA 11.2. $\operatorname{Hom}_{H}(\pi, 1) \cong \oplus_{g \in R \backslash G / H} \operatorname{Hom}_{R \cap g H^{-1}}(\kappa, 1)$.

Proof. Let $\mathcal{F}$ be the $G$-module consisting of all functions $f: G \rightarrow V$ such that $f(h g)=\kappa(h) f(g)$, for all $h \in R$ and $g \in G$, with the $G$-action given by $g \cdot f\left(g^{\prime}\right)=f\left(g^{\prime} g\right)$. Let $\mathcal{F}_{c}$ be the submodule of those $f$ whose support has compact image in $R \backslash G$. In other words, $\mathcal{F}_{c}$ is the representation space of $\pi$. Let $\mathcal{F}_{g, c}$ be the submodule of $f \in \mathcal{F}_{c}$ with support in $\operatorname{RgH}$. There is a canonical isomorphism $\mathcal{F}_{c} \cong \oplus_{g \in R \backslash G / H} \mathcal{F}_{g, c}$ which maps $f \in \mathcal{F}_{c}$ to the collection of functions $\left(f_{g}\right)$, where $f_{g}$ is obtained by restricting $f$ to $R g H$ and then setting $f_{g} \equiv 0$ outside of $R g H$. From this, we obtain an associated canonical isomorphism

$$
\operatorname{Hom}_{H}(\pi, 1)=\operatorname{Hom}_{H}\left(\mathcal{F}_{c}, 1\right) \cong \bigoplus_{g \in R \backslash G / H} \operatorname{Hom}_{H}\left(\mathcal{F}_{g, c}, 1\right)
$$

sending $\Lambda$ to the collection of linear forms $\Lambda_{g}(f)=\Lambda\left(f_{g}\right)$. Next, we observe that there is an isomorphism $\operatorname{Hom}_{H}\left(\mathcal{F}_{g, c}, 1\right) \cong \operatorname{Hom}_{R \cap g H^{-1}}(\kappa, 1)$ such that if $\Lambda \in$ $\operatorname{Hom}_{H}\left(\mathcal{F}_{g, c}, 1\right)$ corresponds to the linear form $\lambda \in \operatorname{Hom}_{R \cap g H^{-1}}(\kappa, 1)$ which satisfies

$$
\Lambda(f)=\sum_{h \in\left(g^{-1} \operatorname{Rg} \cap H\right) \backslash H} \lambda(f(g h)) .
$$

We refer the reader to the proof of Lemma 7 in [8] for further details. 
For the third reduction, we require the following notations. Given $i \in\{1, \ldots$, $r-1\}$, let $\theta^{(i)}=\theta_{i+1}^{E} \cdots \theta_{r}^{E}$ and let $\kappa^{(i)}=\kappa_{i+1} \otimes \cdots \otimes \kappa_{r}$ denote the representation of $R_{i}=E^{\times} B\left(\theta^{(i)}\right)$ on $V^{(i)}=V_{i+1} \otimes \cdots \otimes V_{r}$ associated to $\theta^{(i)}$. Let $H_{i}=H \cap G_{i}$ and let $B^{\prime}(\theta)=B^{\prime}\left(\theta_{r}\right) \cdots B^{\prime}\left(\theta_{1}\right)$, where $B^{\prime}\left(\theta_{i}\right)=B_{m_{i}, i-1}$.

LEMMA 11.3. Assume $g \in G$ and $\operatorname{Hom}_{R \cap g \mathrm{Hg}^{-1}}(\kappa, 1) \neq 0$. If $f_{r}>1$ then $g \in R H$. If $r>1$ and $f_{r}=1$ then $g \in R G_{r-1} H$.

Lemma 11.3 will be strengthened in the next section by removing the technical conditions which apply to the case of $f_{r}=1$.

In spirit, the proof of Lemma 11.3 is similar to an induction on the length $r$ of the Howe factorization of $\theta$, where one verifies the cases of $r=1$ and $r=2$ separately to begin the process. Briefly stated, the argument goes as follows. Assume $r>2$. Suppose $g \in G$ and $\lambda$ is a nonzero element of $\operatorname{Hom}_{R \cap g \mathrm{Hg}^{-1}}(\kappa, 1)$. To show that $g \in R H$, we successively show that $g$ lies in $R G_{1} H, R G_{2} H, \ldots, R G_{r-1} H$ by repeatedly applying the following lemma to $\kappa, \kappa^{(1)}, \ldots, \kappa^{(r-2)}$.

LEMMA 11.4. If $f_{1}>0, g \in G$ and $\operatorname{Hom}_{B^{\prime}(\theta) \cap g H^{-1}}(\kappa, 1) \neq 0$, then $g \in R G_{1} H$.

Lemma 11.4 will be proven at the end of this section. The following proof of Lemma 11.3 relies on the validity of Lemma 11.4.

Proof of Lemma 11.3. Assume $g \in G$ is chosen so that $\operatorname{Hom}_{R \cap g \mathrm{H}^{-1}}(\kappa, 1)$ is nonzero. Since the space $\mathrm{Hom}_{R \cap g \mathrm{Hg}^{-1}}(\kappa, 1)$ only depends on the double coset $R g H$ which $g$ represents, we are free to replace $g$ with another double coset representative when it is convenient to do so. The assumption that $\operatorname{Hom}_{R \cap \mathrm{RHg}^{-1}}(\kappa, 1)$ is nonzero implies that $\operatorname{Hom}_{B^{\prime}(\theta) \cap g g^{-1}}(\kappa, 1)$ is also nonzero. We are assuming that $f_{1}>1$ and thus we may apply Lemma 11.4 to deduce that $g \in R G_{1} H$. If $r=1$ then $R G_{1} H=R H$ and we have the desired result in this case.

Now assume $r>1$. Since $g \in R G_{1} H$, we may assume $g \in G_{1}$. If $f_{2}=1$, we are done, so we will assume $f_{2}>1$. Suppose $\lambda$ is a nonzero element of $\operatorname{Hom}_{B^{\prime}(\theta) \cap g \mathrm{Hg}^{-1}}$ $(\kappa, 1)$. Fix an elementary tensor $v_{1}^{\circ} \otimes \cdots \otimes v_{r}^{\circ}$ which is not in the kernel of $\lambda$. Define a nonzero linear form $\lambda^{(1)} \in \operatorname{Hom}\left(\kappa^{(1)}, 1\right)$ on elementary tensors by

$$
\lambda^{(1)}\left(v_{2} \otimes \cdots \otimes v_{r}\right)=\lambda\left(v_{1}^{\circ} \otimes v_{2} \otimes \cdots \otimes v_{r}\right) .
$$

We assert that $\lambda^{(1)}$ is $\left(B^{\prime}\left(\theta^{(1)}\right) \cap g H_{1} g^{-1}\right)$-invariant. Indeed, this will follow once we show that $\kappa_{1} \mid\left(B_{1,1} \cap g H_{1} g^{-1}\right) \equiv 1$, since $B^{\prime}\left(\theta^{(1)}\right) \subset B_{1,1}$. Note that $\inf \left(\omega_{1}\right)=$ $\omega_{1}=\theta_{1} \circ \operatorname{det}_{1}=1$ on $E^{\times} B_{0,1} \cap g H_{1} g^{-1}$, where the last equality follows from Hilbert's Theorem 90. Therefore, $\kappa_{1}=\kappa_{1}^{\circ}=\hat{\rho}_{1} \circ \xi_{1}$ on $E^{\times} B_{0,1} \cap g H_{1} g^{-1}$. Since $B_{1,1}$ is contained in the kernel of $\xi_{1}$, we conclude that the restriction of $\kappa_{1}$ to $B_{1,1} \cap g H_{1} g^{-1}$ is a multiple of the trivial representation, as claimed.

At this point, we have shown that $\operatorname{Hom}_{B^{\prime}\left(\theta^{(1)}\right) \cap g H_{1} g^{-1}}\left(\kappa^{(1)}, 1\right)$ is nonzero. Since $f_{2}>1$, we may apply Lemma 11.4 to $\kappa^{(1)}$ to see that $g \in R G_{2} H$. Thus we may assume that $g \in G_{2}$. If $r=2$, we are done since then $G_{2}=E^{\times} \subset R H$. Otherwise, we continue 
to apply Lemma 11.4 repeatedly to $\kappa^{(2)}, \kappa^{(3)}, \ldots$ until we conclude $g \in R H$ or, if $f_{r}=1, g \in R G_{r-1} H$.

The rest of this section is devoted to the proof of Lemma 11.4. If $f_{i}>1$, then the representation $\kappa_{i}$ has the property that, on $B^{\prime}\left(\theta_{i}\right) \cdots B^{\prime}\left(\theta_{1}\right)$, it is a multiple of the character defined by

$$
1+x \mapsto\left\langle c_{i}, x\right\rangle, \quad x \in \mathfrak{b}_{m_{i}, i-1}+\cdots+\mathfrak{b}_{m_{1}, 0} .
$$

If $f_{r}=1$, then the restriction of $\kappa_{r}$ to $B^{\prime}(\theta)$ is a multiple of the trivial representation.

Define $c^{(1)} \in E$ by

$$
c^{(1)}= \begin{cases}c_{2}+\cdots+c_{r-1}, & \text { if } f_{r}=1, \\ 0, & \text { if } r=1, \\ c_{2}+\cdots+c_{r}, & \text { if } r \geqslant 2 \text { and } f_{r}>1 .\end{cases}
$$

If $f_{1}>1$ then, since $m_{1} \geqslant m_{i}$ for $2 \leqslant i \leqslant r$, it follows that the restriction of $\kappa$ to $B_{m_{1}}$ is a multiple of the character

$$
1+y \mapsto\left\langle c_{1}+c^{(1)}, y\right\rangle, \quad y \in \mathfrak{b}_{m_{1}} .
$$

Therefore, if $f_{1}>1$ then $\operatorname{Hom}_{B_{m_{1}} \cap g H^{-1}}(\kappa, 1)$ is nonzero precisely when the latter character is trivial on $B_{m_{1}} \cap \mathrm{gHg}^{-1}$. The next lemma translates this statement into a geometric condition on the Lie algebra.

LEMMA 11.5. If $f_{1}>1$ and $\operatorname{Hom}_{B_{m_{1}} \cap g H^{-1}}(\kappa, 1) \neq 0$, then $c_{1}+c^{(1)} \in \mathfrak{b}_{1-m_{1}}+g \mathfrak{h}^{\perp} g^{-1}$.

Proof. Since $\mathfrak{b}_{1-m_{1}}+g \mathfrak{h}^{\perp} g^{-1}=\left(\mathfrak{b}_{m_{1}} \cap g \mathfrak{h} g^{-1}\right)^{\perp}$, it suffices to show that $\left\langle c_{1}+\right.$ $\left.c^{(1)}, z\right\rangle=1$, for all $z \in \mathfrak{b}_{m_{1}} \cap g \mathfrak{h} g^{-1}$. In view of the remarks above, we know that $\left\langle c_{1}+\right.$ $\left.c^{(1)}, y\right\rangle=1$ whenever $1+y \in B_{m_{1}} \cap g H^{-1}$. To make the transition from the algebra $\mathfrak{b}_{m_{1}}$ to the group $B_{m_{1}}$, we again use the Cayley transform $\epsilon(z)=(1+z / 2)(1-z / 2)^{-1}$ as a substitute for the exponential map. Let $\alpha(z)=\left(g \eta g^{*}\right) z^{*}\left(g \eta g^{*}\right)^{-1}$. Then $\alpha(z)=-z$ is the defining condition for $g \mathfrak{h} g^{-1}$ and $\alpha(y)=y^{-1}$ is the defining condition for $\mathrm{gHg}^{-1}$.

Suppose $z \in \mathfrak{b}_{m_{1}}+g \mathfrak{h} g^{-1}$. Then

$$
\alpha(\epsilon(z))=\epsilon(\alpha(z))=\epsilon(-z)=\epsilon(z)^{-1} .
$$

Therefore, $\epsilon(z) \in B_{m_{1}} \cap g \mathrm{Hg}^{-1}$ and thus $\left\langle c_{1}+c^{(1)}, \epsilon(z)-1\right\rangle=1$. Since $c_{1}+c^{(1)} \in$ $\mathfrak{B}^{1-f_{1}} \subset \mathfrak{b}_{f_{1}}^{\perp} \subset \mathfrak{b}_{2 m_{1}}^{\perp}$ and since $\epsilon(z) \equiv 1+z$ modulo $\mathfrak{b}_{2 m_{1}}$, we have $\left\langle c_{1}+c^{(1)}, z\right\rangle=$ $\left\langle c_{1}+c^{(1)}, \epsilon(z)-1\right\rangle=1$. Our claim follows.

LEMMA 11.6. If $f_{1}>1$, then $\operatorname{Ad} B_{\ell_{1}}\left(c_{1}+c^{(1)}+\mathfrak{b}_{1,1-m_{1}}\right)=c_{1}+c^{(1)}+\mathfrak{b}_{1-m_{1}}$.

Proof. By Lemma 6 of [15], we have

$$
\text { Ad } B_{\ell_{1}}\left(\gamma_{1}+\mathfrak{b}_{1,1-m_{1}}\right)=\gamma_{1}+\mathfrak{b}_{1-m_{1}} \text {. }
$$

Our assertion is now a consequence of the fact that $c_{1}+c^{(1)} \in \gamma_{1}+\mathfrak{B}^{2-f_{1}}$. 
LEMMA 11.7. If $g \in G$ and $g \sigma_{\eta}(g) \in G_{i}$ then $g \in G_{i} H$.

Proof. Let $Z^{1}=\left\{g \in G_{i}: g=\sigma_{\eta}(g)\right\}$ and $B^{1}=\left\{g \sigma_{\eta}(g): g \in G_{i}\right\}$. From the theory of Hermitian matrices over local fields, we know that an element of $Z^{1}$ lies in $B^{1}$ precisely when its discriminant is a norm. Now suppose $g \in G$ and $g \sigma_{\eta}(g) \in G_{i}$. Then $g \sigma_{\eta}(g)$ is an element of $Z^{1}$ whose discriminant is a norm. Thus $g \sigma_{\eta}(g)=g_{1} \sigma_{\eta}\left(g_{1}\right)$, for some $g_{1} \in G_{i}$. Now $g_{1}^{-1} g=\sigma_{\eta}\left(g_{1}^{-1} g\right)$ implies $g \in g_{1} H \subset G_{i} H$, which proves our assertion.

LEMMA 11.8. If $f_{1}>1$ and $c_{1}+c^{(1)} \in \mathfrak{b}_{1-m_{1}, 1}+g \mathfrak{h}^{\perp} g^{-1}$ then $g \in G_{1} H$.

Proof. Let $z \in \mathfrak{b}_{1-m_{1}, 1}$ and $x \in \mathfrak{h}^{\perp}$ be such that $c_{1}+c^{(1)}+z=g x g^{-1}$. From $\sigma_{\eta}\left(c_{1}+c^{(1)}\right)=c_{1}+c^{(1)}$ and $\sigma_{\eta}(x)=x$, we see that

$$
c_{1}+c^{(1)}+z=g \sigma_{\eta}(g)\left(c_{1}+c^{(1)}+\sigma_{\eta}(z)\right)\left(g \sigma_{\eta}(g)\right)^{-1} .
$$

Note that $c_{1}+c^{(1)}+z \in \gamma_{1}+\mathfrak{b}_{2-f_{1}, 1}$. Similarly for $c_{1}+c^{(1)}+\sigma_{\eta}(z)$. We can apply Lemma 8 of [15] to conclude that $\gamma_{1}=g \sigma_{\eta}(g)\left(\gamma_{1}\right)\left(g \sigma_{\eta}(g)\right)^{-1}$. As $\gamma_{1}$ generates $E_{1} / F$, this condition is equivalent to $g \sigma_{\eta}(g) \in G_{1}$, that is, $g_{l}\left(g^{-1}\right) \in G_{1}$. Now applying Lemma 11.7, we deduce $g \in G_{1} H$.

Proof of Lemma 11.4. Assume $f_{1}>1$ and $\operatorname{Hom}_{B^{\prime}(\theta) \cap g \mathrm{Hg}^{-1}}(\kappa, 1) \neq 0$. Then by Lemma 11.5 and Lemma 11.6, there exists $b \in B_{\ell_{1}}$ such that $c_{1}+c^{(1)} \in \mathfrak{b}_{1,1-m_{1}}+$ $b g \mathfrak{h}^{\perp}(b g)^{-1}$. By Lemma 11.8, $b g \in G_{1} H$. Hence, $g \in B_{\ell_{1}} G_{1} H \subset R G_{1} H$.

\section{The Case of $f_{r}=1$}

According to the strategy outlined in Section 11, the computation of the space $\operatorname{Hom}_{H}(\pi, 1)$ of $H$-invariant linear forms associated to the supercuspidal representation $\pi=\operatorname{Ind}_{R}^{G}(\kappa)$ reduces to a matter of computing the spaces $\operatorname{Hom}_{R \cap g g^{-1}}(\kappa, 1)$ as $g$ ranges over a set of representatives for $R \backslash G / H$. This section is devoted to demonstrating that all double cosets other than $R H$ are extraneous. We prove:

PROPOSITION 12.1. If $g \in G$ and $\operatorname{Hom}_{R \cap g g^{-1}}(\kappa, 1) \neq 0$ then $g \in R H$.

The proof appears at the end of the section after a substantial amount of preparation. When $f_{r}>1$ then assertion in Proposition 12.1 has been shown in Section 11 in Lemma 11.3. Therefore, throughout this section we assume $f_{r}=1$. There are two cases which are considered separately: the case of $r=1$ and the case of $r \geqslant 2$. In light of Lemma 11.3, when $r \geqslant 2$, it suffices to show that if $g \in G_{r-1}$ and $\operatorname{Hom}_{R \cap g g^{-1}}(\kappa, 1) \neq 0$ then $g$ must lie in $R H$. The cases $r=1$ and $r \geqslant 2$ will subdivide further depending on the image of $R \cap g \mathrm{Hg}^{-1}$ over the residue field. For most $g$, the corresponding group over the residue field will be 'superunipotent' in the sense that it contains the unipotent radical of a parabolic subgroup. If it is not superunipotent and if $g \notin R H$ then it turns out that we obtain a symplectic group over the residue field. The parallel roles played by unipotent radicals and symplectic groups is reminiscent of a similar phenomenon which links the theories of symplectic models and Whittaker/Gelfand/Graev models. This phenomenon was first observed by 
Klyachko [21], in the context of finite fields, then studied by Heumos and Rallis [14] over local fields and Jacquet and Rallis [18] over global fields.

Once Proposition 12.1 is established, it will remain to study the spaces $\operatorname{Hom}_{R \cap H}(\kappa, 1)$. In Section 13, we show that these spaces factor into spaces $\operatorname{Hom}_{R \cap H}\left(\kappa_{i}, \delta_{i}\right)$. When $f_{i}>1$, $\operatorname{Hom}_{R \cap H}\left(\kappa_{i}, \delta_{i}\right)$ has already been described in Proposition 10.1. A secondary focus of this section is to provide the analogue of Proposition 10.1 when $f_{i}=1$.

Having described our objectives, let us now attend to the case of $f_{1}=1$. The main results are taken from [9] with one enhancement which allows us to prove that the dimension of $\operatorname{Hom}_{H}(\pi, 1)$ is one. The work in [9] draws on [8] as well as results of Gow [7], Klyachko [21] and Lusztig [23] involving the representation theory of finite groups of Lie type.

Recall that $q_{0}$ and $q_{0}^{\prime}$ are the orders of the residue fields $k_{0}$ and $k_{0}^{\prime}$ of $F$ and $F^{\prime}$, respectively. If $F / F^{\prime}$ is unramified, that is, if $q_{0}=q_{0}^{\prime 2}$, then $\operatorname{GL}\left(n, k_{0}\right)$ contains the standard unitary group $\mathrm{U}\left(n, q_{0}\right)$. When $F / F^{\prime}$ is ramified, we will consider the standard orthogonal group $\mathrm{O}\left(n, q_{0}\right)$ and, when $n$ is even, the orthogonal group $\mathrm{O}^{\prime}\left(n, q_{0}\right)$ associated to the diagonal matrix diag $(1, \ldots, 1, \delta)$, where $\delta$ is some fixed nonsquare element of $k_{0}^{\prime x}$. When $F / F^{\prime}$ is ramified and $n$ is even, we also consider the symplectic group $\operatorname{Sp}\left(n, q_{0}\right)$. We will say that a subgroup of $\operatorname{GL}\left(n, k_{0}\right)$ is superunipotent if it contains the unipotent radical of a proper parabolic subgroup of $\mathrm{GL}\left(n, k_{0}\right)$. The significance of this notion is that when $S$ is superunipotent and $\rho$ is an irreducible cuspidal representation of $\operatorname{GL}\left(n, k_{0}\right)$, then the space of $\pi$ cannot contain any nonzero vectors which are fixed by $S$.

When $f_{1}=1, E / F$ must be unramified, $\theta=\theta_{1}$ and $\theta \mid \mathfrak{D}_{E}^{\times}$is the inflation of a character $\chi$ of the multiplicative group of the residue field $k_{1}$ of $E$. In addition, $\theta=\theta^{\prime} \circ N_{E / E^{\prime}}$ for some quasicharacter $\theta^{\prime}$ of $E^{\prime \times}$ or, equivalently, $\theta$ is trivial on $\mathrm{U}\left(1, E / E^{\prime}\right)$. The group $R=E^{\times} B$ is generated by a prime element $\varpi_{0}$ of $F$ and the elements of $B$. Deligne-Lusztig's construction associates to $\chi$ a cuspidal representation $\left(\rho_{1}, V\right)$ of $\mathrm{GL}\left(n, k_{0}\right)$ which we sometimes view as a representation of $B$ via the isomorphism $B / B_{1} \cong \mathrm{GL}\left(n, k_{0}\right)$. The representation $\kappa$ of $R$ on $V$ is defined by $\kappa\left(\varpi^{j} b\right)=\theta(\varpi)^{j} \rho_{1}(b)$, for all $j \in Z$ and $b \in B$. Suppose we are given $g \in G$. The determinant of any element of $g \mathrm{Hg}^{-1}$ has modulus one and thus $\mathrm{R} \cap \mathrm{gHg} \mathrm{g}^{-1}=\mathrm{B} \cap \mathrm{gHg} \mathrm{g}^{-1}$. If $U_{g}$ is the image of $B \cap g_{H^{-1}}$ in $\operatorname{GL}(n, q)$ under the isomorphism $B / B_{1} \cong \mathrm{GL}\left(n, k_{0}\right)$, then $\operatorname{Hom}_{R \cap g H^{-1}}(\kappa, 1)=\operatorname{Hom}_{U_{g}}\left(\rho_{1}, 1\right)$ and

$$
\operatorname{Hom}_{H}(\pi, 1) \cong \prod_{g \in R \backslash G / H} \operatorname{Hom}_{U_{g}}\left(\rho_{1}, 1\right) .
$$

PROPOSITION 12.2. Assume $f_{1}=1$.

(1) $\operatorname{dim} \operatorname{Hom}_{H}(\pi, 1)=1$.

(2) Suppose $F / F^{\prime}$ is unramified. Then $n$ must be odd. If $g \in R H$, then $U_{g}$ is conjugate to $U\left(n, q_{0}\right)$ and $\operatorname{dim}_{\operatorname{Hom}_{U_{g}}}\left(\rho_{1}, 1\right)=1$. Otherwise, if $g \in G-R H$, then $U_{g}$ is superunipotent and $\operatorname{Hom}_{U_{g}}\left(\rho_{1}, 1\right)=0$. 
(3) Suppose $F / F^{\prime}$ is ramified. If $g \in R H$ then $U_{g}$ is conjugate to $\mathrm{O}\left(n, q_{0}\right)$ unless $n$ is even and $\operatorname{det} \eta \in F^{\times}-N_{F / F^{\prime}}\left(F^{\times}\right)$, in which case $U_{g}$ is conjugate to $\mathrm{O}^{\prime}\left(n, q_{0}\right)$. In either of these cases, $\operatorname{dim}_{H_{U_{g}}}\left(\rho_{1}, 1\right)=1$. If $g \in G-R H$, then $U_{g}$ is either superunipotent or conjugate to $\mathrm{Sp}\left(n, q_{0}\right)$, if $n$ is even. In these cases, $\operatorname{Hom}_{U_{g}}\left(\rho_{1}, 1\right)=0$.

Proof. It follows from Lemma 6.2 that if $F / F^{\prime}$ is unramified $n$ must be odd. Combining this observation with Theorem 1 of [9] proves that $\operatorname{dim} \operatorname{Hom}_{H}(\pi, 1) \leqslant 1$. Now suppose $F / F^{\prime}$ is unramified. To deduce (1) in this case as well as (2), one applies Lemmas 4 and 5 of [9] together with Theorem 2.4 of [7]. In applying Gow's Theorem 2.4 , one first observes that the character of $\rho_{1}$ is Galois invariant and thus, according to Gow's result, $\rho_{1}$ embeds uniquely in the representation of $\operatorname{GL}\left(n, k_{0}\right)$ induced from the trivial representation of $U_{1}$. Then Frobenius reciprocity implies that the dimension of $\operatorname{Hom}_{U_{1}}\left(\rho_{1}, 1\right)$ is one. The remaining assertions regarding the case in which $F / F^{\prime}$ is ramified follow from Theorems 1 and 2 of [8].

For the remainder of the section, assume $r>1$ and $f_{r}=1$. We need the following analogue of Proposition 10.1:

PROPOSITION 12.3. Assume $r>1$ and $f_{r}=1$ and let $\vartheta_{r}=\prod_{i=1}^{r-1} \vartheta_{i}$, with $\vartheta_{i}$ chosen as in Proposition 10.1. Then the spaces $\operatorname{Hom}_{R \cap H}\left(\kappa_{r}, \vartheta_{r}\right)$ and $\operatorname{Hom}_{B_{0, r-1} \cap H}\left(\kappa_{r}, 1\right)$ are identical and have dimension one.

Proof. When $\vartheta_{r}=1$, the proof of our assertions is identical to the proof of (2) and (3) of Proposition 12.2. Therefore, we may as well assume that $\vartheta_{r} \neq 1$. Hence, $E / E^{\prime}$ is ramified and $n_{r-1}$ is even. Recall from Section 9, that $\mathcal{B}=(R \cap H) /\left(R \cap H \cap B_{1}\right)$ is isomorphic to a certain orthogonal group $\mathrm{O}\left(\tilde{\eta}, k_{r-1}\right)$ in $\mathcal{G}=(R \cap B) /\left(R \cap B_{1}\right) \cong$ $\mathrm{GL}\left(n_{r-1}, k_{r-1}\right)$. The character $\vartheta_{r}$ may be regarded as a character of $\mathcal{B}$. In fact, it must be the character which is 1 on the special orthogonal group and -1 on its complement, since this is the unique nontrivial quadratic character of $\mathcal{B}$. We have

$$
\operatorname{Hom}_{R \cap H}\left(\kappa_{r}, \vartheta_{r}\right)=\operatorname{Hom}_{\mathcal{B}}\left(\rho_{r}, \vartheta_{r}\right)=\operatorname{Hom}_{\mathcal{B}}\left(\rho_{r} \otimes \vartheta_{r}, 1\right)
$$

and, since $n_{r-1}$ is even, $\vartheta_{r}(-1)=1$. Now, the representation $\rho_{r} \otimes \vartheta_{r}$ is an irreducible cuspidal representation whose central character is trivial at -1 . Therefore, Theorem 2 of [8] implies $\operatorname{dim} \operatorname{Hom}_{\mathcal{B}}\left(\rho_{r} \otimes \vartheta_{r}, 1\right)=1$.

Our next task is to recall from [9] the Jacobowitz/O'Meara description of the double coset space $B_{0, r-1} \backslash G_{r-1} / H_{r-1}$. Actually, the double coset space $R_{r-1} \backslash G_{r-1} / H_{r-1}$ is more directly relevant to the problems we are studying, however, we save some effort by being consistent with [16] and [9] and working with $B_{0, r-1} \backslash G_{r-1} / H_{r-1}$ instead. As $f_{r}=1$, both $E / E_{r-1}$ and $E^{\prime} / E_{r-1}^{\prime}$ must be unramified according to Lemma 6.2 (ii) and, therefore, we can apply Lemma 2.3 to arrange that the embedding of $E$ in $\mathfrak{g}_{r-1}$ is associated to an integral basis of $E / E_{r-1}$. It follows that $B_{0, r-1}=$ $\operatorname{GL}\left(n_{r-1}, \mathfrak{D}_{r-1}\right)$. This will allow us to describe the double coset space $B_{0, r-1} \backslash G_{r-1} /$ $H_{r-1}$ exactly as in [9]. 
Let $\mathcal{A}$ be the set of sequences $\alpha=\left(\alpha_{1}, \ldots, \alpha_{m}\right)$ with $\alpha_{i}=\left(a_{i}, b_{i}, \epsilon_{i}\right)$ satisfying the following properties:

(i) $a_{1}>\cdots>a_{m}$ is a decreasing sequence of integers.

(ii) $b_{1}+\cdots+b_{m}=n_{r-1}$ is a partition of $n_{r-1}$ by positive integers.

(iii) If $E / E^{\prime}$ is unramified then $\epsilon_{i}=1$.

(iv) If $E / E^{\prime}$ is ramified and $a_{i}$ is odd then $b_{i}$ must be even and $\epsilon_{i}=1$.

(v) If $E / E^{\prime}$ is ramified and $a_{i}$ is even then $\epsilon_{i} \in\{1, \delta\}$, where $\delta$ is some fixed unit in $E_{r-1}^{\prime}$ whose image in the residue field of $E_{r-1}^{\prime}$ is a nonsquare.

Assume $\varpi_{r-1} \in E^{\prime}{ }_{r-1}$, if $E / E^{\prime}$ is unramified, and $\varpi_{r-1}^{2} \in E_{r-1}^{\prime}$, if $E / E^{\prime}$ is ramified. Given $\alpha=\left(\alpha_{1}, \ldots, \alpha_{m}\right) \in \mathcal{A}$, let $\ell(\alpha)=m$, and for each index $i$ define a Hermitian matrix $\varpi^{\alpha_{i}} \in \mathrm{GL}\left(b_{i}, E_{r-1}\right)$ as follows:

(a) If $E / E^{\prime}$ is unramified then $\varpi^{\alpha_{i}}=\varpi_{r-1}^{a_{i}} 1_{b_{i}}$.

(b) If $E / E^{\prime}$ is ramified and $a_{i}$ is odd then $\varpi^{\alpha_{i}}=\varpi_{r-1}^{a_{i}}\left(\left(\begin{array}{cc}0 & 1 \\ -1 & 0\end{array}\right) \oplus \cdots \oplus\left(\left(\begin{array}{cc}0 & 1 \\ -1 & 0\end{array}\right)\right)\right.$.

(c) If $E / E^{\prime}$ is ramified and $a_{i}$ is even then $\varpi^{\alpha_{i}}=\varpi_{r-1}^{a_{i}}\left(1 \oplus \cdots \oplus 1 \oplus \epsilon_{i}\right)$.

Here, if $X$ and $Y$ are square matrices, $X \oplus Y$ denotes the square matrix which, in block form, is written as $\left(\begin{array}{ll}X & 0 \\ 0 & Y\end{array}\right)$. Given $\alpha \in \mathcal{A}$, define a Hermitian matrix by

$$
\varpi^{\alpha}=\varpi^{\alpha_{1}} \oplus \cdots \oplus \varpi^{\alpha_{m}}
$$

and let

$$
H_{\alpha}=\left\{g \in G_{r-1}: g \varpi^{\alpha} g^{*}=\varpi^{\alpha}\right\} .
$$

The following result is due to Jacobowitz [16]. It describes the orbits of the action of $B_{0, r-1}$ on the space $\mathcal{X}=\left\{x \in G_{r-1}: x^{*}=x\right\}$, where the action is given by $b \cdot x=b x b^{*}$.

LEMMA 12.4. Assume $f_{r}=1$. The set $\left\{\varpi^{\alpha}: \alpha \in \mathcal{A}\right\}$ is a set of representatives for the $B_{0, r-1}$-orbits in $\mathcal{X}$.

Consider now the action of $G_{r-1}$ on $\mathcal{X}$ by $g \cdot x=g x g^{*}$. For this action, there are two orbits. The orbit of a given $x \in \mathcal{X}$ is determined by the class of $\operatorname{det}_{r-1}(x)$ in $E_{r-1}^{\prime \times} / N_{E_{r-1} / E_{r-1}^{\prime}}\left(E_{r-1}^{\times}\right)$. Let $\mathcal{X}_{\eta}$ be the $G_{r-1}$-orbit of $\eta$ and let $\mathcal{A}_{\eta}=\{\alpha \in \mathcal{A}$ : $\left.\varpi^{\alpha} \in \mathcal{X}_{\eta}\right\}$. For each $\alpha \in \mathcal{A}_{\eta}$, assume we have fixed $g_{\alpha} \in G_{r-1}$ such that $g_{\alpha} \eta g_{\alpha}^{*}=\varpi^{\alpha}$.

COROLLARY 12.5. The set $\left\{g_{\alpha}: \alpha \in \mathcal{A}_{\eta}\right\}$ is a set of representatives for the double coset space $B_{0, r-1} \backslash G_{r-1} / H_{r-1}$.

Proof. The map $g \mapsto g \eta g^{*}$ gives a bijection between $G_{r-1} / H_{r-1}$ and $\mathcal{X}_{\eta}$. This map is $B_{0, r-1}$-equivariant, where $B_{0, r-1}$ acts by left translations on $G_{r-1} / H_{r-1}$ and by $b \cdot x=b x b^{*}$ on $\mathcal{X}_{\eta}$. Therefore, we obtain a bijection between $B_{0, r-1} \backslash G_{r-1} / H_{r-1}$ and the set of $B_{0, r-1}$-orbits in $\mathcal{X}_{\eta}$. We see that the set $\left\{g_{\alpha}: \alpha \in \mathcal{A}_{\eta}\right\}$ is a set of representatives for the double coset space $B_{0, r-1} \backslash G_{r-1} / H_{r-1}$. Applying Lemma 12.4 now finishes the proof. 
We return now to the proof of Proposition 12.1. We are reduced to considering the case in which $r \geqslant 2$ and $f_{r}=1$. Moreover, we only need to show that if $g \in G_{r-1}-$ $R_{r-1} H_{r-1}$ then $\operatorname{Hom}_{R_{r-2} \cap g H^{-1}}(\kappa, 1)=0$. Corollary 12.5 allows us to assume that $g=g_{\alpha}$, for some $\alpha \in \mathcal{A}_{\eta}$.

LEMMA 12.6. If $\alpha \in \mathcal{A}_{\eta}, g_{\alpha} \in G_{r-1}-R_{r-1} H_{r-1}$ and $\ell(\alpha)=1$, then $E / E^{\prime}$ is ramified, $n_{r-1}$ is even and $\varpi^{\alpha}=\varpi_{r-1}^{a}\left(\left(\begin{array}{cc}0 & 1 \\ -1 & 0\end{array}\right) \oplus \cdots \oplus\left(\begin{array}{cc}0 & 1 \\ -1 & 0\end{array}\right)\right)$ for some odd integer a.

Proof. Assume $\ell(\alpha)=1$. Suppose $E / E^{\prime}$ is unramified. Then, according to the Assumption in Section 3, either $\eta=1$ or $\eta=\varpi_{r-1}^{\prime}$. On the other hand, $\varpi^{\alpha}=\varpi_{r-1}^{a}$, for some integer $a$. Using the $R_{r-1}$-equivariant bijection $g \mapsto g \eta g^{*}$ between $G_{r-1} / H_{r-1}$ and $\mathcal{X}_{\eta}$, we see that $g_{\alpha} \in R_{r-1} H_{r-1}$ precisely when $\varpi^{\alpha}=g_{\alpha} \eta g_{\alpha}^{*}$ is in the $R_{r-1}$-orbit of $\eta$. But we have $\varpi_{r-1}^{s} \eta\left(\varpi_{r-1}^{s}\right)^{*}=\varpi^{\alpha}$, where $s$ is the least integer greater than or equal to $a / 2$. Therefore, if $E / E^{\prime}$ is unramified and $\ell(\alpha)=1$ then $R_{r-1} g_{\alpha} H_{r-1}=R_{r-1} H_{r-1}$.

Now suppose $E / E^{\prime}$ is ramified. Let say that $\varpi^{\alpha}$ is of symplectic type if it has the form

$$
\varpi_{r-1}^{a}\left(\left(\begin{array}{cc}
0 & 1 \\
-1 & 0
\end{array}\right) \oplus \cdots \oplus\left(\begin{array}{cc}
0 & 1 \\
-1 & 0
\end{array}\right)\right)
$$

for some odd integer $a$. According to the Assumption in Section 3, either $\eta=1$ or $\eta$ is a nonsquare root of unity. It follows from the odd parity of $a$ in the definition of symplectic type that $\eta$ cannot lie in the $R_{r-1}$-orbit of a matrix of symplectic type. Now assume we have fixed $\varpi^{\alpha}$ with $\ell(\alpha)=1$ which is not of symplectic type. We must show that $g_{\alpha} \in R_{r-1} H_{r-1}$ or, equivalently, $\varpi^{\alpha}$ lies in the $R_{r-1}$-orbit of $\eta$. According to Lemma 12.4, the $B_{0, r-1}$-orbits of $\eta$ and $\varpi^{\alpha}$ must contain either 1 or $1 \oplus \cdots \oplus 1 \oplus \delta$. Since $\operatorname{det}_{r-1} \eta=\operatorname{det}_{r-1} \varpi^{\alpha}$, either both $\eta$ and $\varpi^{\alpha}$ lie in the $B_{0, r-1^{-}}$ orbit of 1 or they both lie in the orbit of $1 \oplus \cdots \oplus 1 \oplus \delta$. In either case, our assertion follows.

Assume $\alpha \in \mathcal{A}_{\eta}$ and $\ell(\alpha)>1$. We will associate to $\alpha$ a group $N_{\alpha}$ which is the unipotent radical of a maximal parabolic subgroup of $G$ and then we expose an intimate relationship between $N_{\alpha}$ and $H_{\alpha}$. This is used to correlate $H_{\alpha}$-invariant linear forms with $N_{\alpha}$-invariant linear forms. Arguing that the existence of nonzero $N_{\alpha}$-invariant linear forms contradicts the supercuspidality of $\kappa$, we are able to rule out the existence of nonzero $H_{\alpha}$-invariant linear forms when $\ell(\alpha)>1$.

The exact definition of $N_{\alpha}$ is as follows. Let

$$
v_{1}=b_{m}\left[E_{r-1}: F\right] \quad \text { and } \quad v_{2}=\left(n_{r-1}-b_{m}\right)\left[E_{r-1}: F\right] .
$$

Then $N_{\alpha}$ consists of the matrices in $G$ with block matrix form $\left(\begin{array}{cc}1_{1} & 0 \\ u & 1\end{array}\right)$, where $u$ is a $v_{1} \times v_{2}$ matrix with entries in $F$. Let $\mathfrak{n}_{\alpha}$ be the Lie algebra of $N_{\alpha}$, that is, $N_{\alpha}=1+\mathfrak{n}_{\alpha}$. Note that $\mathfrak{n}_{\alpha} \subset E_{r-1}^{\perp}$.

We associate to each $y=\left(\begin{array}{ll}0 & 0 \\ u & 0\end{array}\right) \in \mathfrak{n}_{\alpha}$ an element $\hat{y}=y-\varpi^{\alpha} y^{*}\left(\varpi^{\alpha}\right)^{*}$ in the Lie algebra 


$$
\mathfrak{h}_{\alpha}=g_{\alpha} \mathfrak{h} g_{\alpha}^{-1}=\left\{z \in \mathfrak{g}: \varpi^{\alpha} z^{*}\left(\varpi^{\alpha}\right)^{-1}=-z\right\}
$$

of $H_{\alpha}$. The map $y \in \mathfrak{n}_{\alpha} \mapsto \hat{y} \in \mathfrak{h}_{\alpha}$ has a number of interesting properties which are summarized in the following lemma:

LEMMA 12.7. Assume $f_{r}=1, \alpha \in \mathcal{A}_{\eta}$ and $\ell(\alpha)>1$.

(1) If $y \in \mathfrak{n}_{\alpha}$ then

$y-\hat{y}=\varpi^{\alpha} y^{*}\left(\varpi^{\alpha}\right)^{-1}=\left(\begin{array}{cc}0 & \varpi^{\alpha^{\prime}} u^{*}\left(\varpi^{\alpha_{m}}\right)^{-1} \\ 0 & 0\end{array}\right) \in \mathfrak{n}_{\alpha}^{*}$,

where $\alpha^{\prime}=\left(\alpha_{1}, \ldots, \alpha_{m-1}\right)$.

(2) If $y \in \mathfrak{n}_{\alpha} \cap \mathfrak{b}_{j}$ then $\hat{y} \in \mathfrak{h}_{\alpha} \cap \mathfrak{b}_{j}$ and $y-\hat{y} \in \mathfrak{n}_{\alpha}^{*} \cap \mathfrak{b}_{j+1}$.

(3) If $y \in \mathfrak{n}_{\alpha} \cap \mathfrak{g}_{i}^{\perp}$ then $\hat{y} \in \mathfrak{h}_{\alpha} \cap \mathfrak{g}_{i}^{\perp}$.

(4) If $1 \leqslant i \leqslant r-1$ and $1+y \in N_{\alpha} \cap J_{i}$ then $1+\hat{y} \in H_{\alpha} \cap J_{i}$ and $(1+y)(1+\hat{y})^{-1} \in$ $J_{i}^{\prime \prime}$.

Proof. The verifications of all of the above assertions are straightforward, except for (2) which follows from the identity $y-\hat{y}=\varpi_{r-1}^{a_{m-1}-a_{m}} \gamma_{1} y^{*} \gamma_{2}$, where

$$
\begin{aligned}
& \gamma_{1}=\left(\begin{array}{cc}
\varpi_{r-1}^{-a_{m-1}} \varpi^{\alpha^{\prime}} & 0 \\
0 & 1_{b_{m}}
\end{array}\right), \\
& \gamma_{2}=\left(\begin{array}{cc}
1_{b_{m}^{\prime}} & 0 \\
0 & \varpi_{r-1}^{a_{m}}\left(\varpi^{\alpha_{m}}\right)^{-1}
\end{array}\right) \in B_{0, r-1}=\mathrm{GL}\left(n_{r-1}, \bigcirc_{r-1}\right) .
\end{aligned}
$$

LEMMA 12.8. $R \cap N_{\alpha}=\left(B_{0, r-1} \cap N_{\alpha}\right)\left(J_{r-1} \cap N_{\alpha}\right) \cdots\left(J_{1} \cap N_{\alpha}\right)$.

Proof. If $i \in\{1, \ldots, r\}$ then the identity

$$
\mathfrak{g}_{i-1}=\mathfrak{g l}\left(n_{i}, \mathfrak{g l}\left(\left[E_{i}: E_{i-1}\right], E_{i-1}\right)\right)
$$

allows us to view the elements of $\mathrm{g}_{i-1}$ as block matrices. It is then evident that we have the decomposition $\mathfrak{g}_{i-1}=\mathfrak{g}_{i} \oplus\left(\mathfrak{g}_{i-1} \cap \mathfrak{g}_{i}^{\perp}\right)$. Applying this repeatedly, we obtain

$$
\mathfrak{g}=\mathfrak{g}_{1}^{\perp} \oplus\left(\mathfrak{g}_{1} \cap \mathfrak{g}_{2}^{\perp}\right) \oplus \cdots \oplus \mathfrak{g}_{r-1} .
$$

We can express each $x \in \mathfrak{g}$ as $x=x_{0}+\cdots+x_{r-1}$ according to this decomposition. Since $\mathfrak{n}_{\alpha}$ is defined in a way which respects the various block matrix decompositions, we know that if $x \in \mathfrak{n}_{\alpha}$ then all of the components $x_{0}, \ldots, x_{r-1}$ will also lie in $\mathfrak{n}_{\alpha}$.

Next, we observe that $\mathfrak{b}_{\ell_{i+1}, i}=\mathfrak{b}_{\ell_{i+1}, i+1} \oplus\left(\mathfrak{b}_{\ell_{i+1}, i} \cap \mathfrak{g}_{i+1}^{\perp}\right)$, whenever $i=0, \ldots, r-1$, and $R \cap B \subset \mathfrak{b}_{0, r-1}+\mathfrak{b}_{\ell_{r-1}, r-2}+\cdots+\mathfrak{b}_{\ell_{1}, 0}$. Combining these two facts, yields

$$
R \cap B \subset \mathfrak{b}_{0, r-1}+\left(\mathfrak{b}_{\ell_{r-1}, r-2} \cap \mathfrak{g}_{r-1}^{\perp}\right)+\cdots+\left(\mathfrak{b}_{\ell_{1}, 0} \cap \mathfrak{g}_{1}^{\perp}\right) .
$$

Now suppose $y=1+x \in N_{\alpha} \cap R=N_{\alpha} \cap R \cap B$. Using Equation (**), we obtain an expression $y=y_{0}+\cdots+y_{r-1}$, where $y_{r-1} \in \mathfrak{b}_{0, r-1}$ and, otherwise, $y_{i} \in \mathfrak{b}_{\ell_{i+1}, i}$ 
$\cap \mathfrak{g}_{i+1}^{\perp}$. The element $x$ has a similar decomposition $x=x_{0}+\cdots+x_{r-1}$, with $x_{0}=y_{0}-1 \in \mathfrak{b}_{0, r-1}$ and, otherwise, $x_{i}=y_{i} \in \mathfrak{b}_{\ell_{i+1}, i} \cap \mathfrak{g}_{i+1}^{\perp}$. This decomposition of $x$ must be identical to the decomposition in Equation $(*)$, according to the uniqueness of the latter decomposition. Therefore, $x_{r-1} \in \mathfrak{n}_{\alpha} \cap \mathfrak{b}_{0, r-1}$ and, for $i \neq r-1$, we have $x_{i} \in \mathfrak{n}_{\alpha} \cap \mathfrak{b}_{\ell_{i+1}, i} \cap \mathfrak{g}_{i+1}^{\perp}$. This implies:

$$
\begin{aligned}
1+x & =1+x_{0}+\cdots+x_{r-1}=\left(1+x_{0}\right) \cdots\left(1+x_{r-1}\right) \\
& \in\left(N_{\alpha} \cap J_{1}\right) \cdots\left(N_{\alpha} \cap J_{r-1}\right)\left(N_{\alpha} \cap B_{0, r-1}\right)
\end{aligned}
$$

and our claim follows. (Note that if $1+u, 1+v \in N_{\alpha}$ then we have $(1+u)$ $(1+v)=1+u+v$, using block matrix multiplication or the fact that $N_{\alpha}$ is the unipotent radical of a maximal parabolic subgroup of $G$.)

LEMMA 12.9. If $i$ and $j$ are distinct integers with $1 \leqslant i \leqslant r-1$ and $1 \leqslant j \leqslant r$ then the restricted representations $\kappa_{j} \mid H_{\alpha} \cap J_{i}$ and $\kappa_{j} \mid N_{\alpha} \cap J_{i}$ are multiples of the trivial representations of $H_{\alpha} \cap J_{i}$ and $N_{\alpha} \cap J_{i}$.

Proof. If $j=r$ we have $\kappa_{r} \mid J_{i} \equiv 1$, since $J_{i} \subset B_{1}$. So let us assume $j \neq r$. Suppose $j>i$. Then $\kappa_{j} \mid J_{i}$ is a multiple of the character of $J_{i}$ defined by $x \mapsto\left\langle c_{j}, x\right\rangle$. Since $c_{j} \in E^{\prime}{ }_{r-1}$ and $\mathfrak{n}_{\alpha} \subset E_{r-1}^{\perp}$, we have $\kappa_{j} \mid N_{\alpha} \cap J_{i} \equiv 1$. On the other hand, from Lemma 8.1 (v) we have $c_{j} \in\left(\mathfrak{h}_{\alpha}+\mathfrak{b}_{f_{j}}\right)^{\perp} \cap E$ and, since $H_{\alpha} \cap J_{i} \subset \epsilon_{i}\left(\mathfrak{h}_{\alpha} \cap \mathfrak{b}_{\ell_{i}, i-1}\right) \subset 1+\left(\mathfrak{h}_{\alpha} \cap\right.$ $\left.\mathfrak{b}_{\ell_{i}, i-1}\right)+\mathfrak{b}_{2 \ell_{i}, i-1} \subset \mathfrak{h}_{\alpha}+\mathfrak{b}_{f_{j}}$, it follows that $\kappa_{j} \mid H_{\alpha} \cap J_{i} \equiv 1$. Suppose now that $j<i$. Then $\kappa_{j} \mid J_{i}$ is a multiple of $\theta_{j} \circ \operatorname{det}_{j}$. Since $\operatorname{det}_{j}\left(N_{\alpha} \cap J_{i}\right)=1$, we have $\kappa_{j} \mid N_{\alpha} \cap J_{i} \equiv 1$ and, since $\operatorname{det}_{j}\left(H_{\alpha} \cap J_{i}\right) \subset U\left(1, E_{j} / E_{j}^{\prime}\right)$, we have $\kappa_{j} \mid H_{\alpha} \cap J_{i} \equiv 1$.

LEMMA 12.10. If $\ell(\alpha)>1$ then $\operatorname{Hom}_{H_{\alpha} \cap R}(\kappa, 1) \subset \operatorname{Hom}_{N_{\alpha} \cap R}(\kappa, 1)$.

Proof. Suppose $\lambda \in \operatorname{Hom}_{H_{\alpha} \cap R}(\kappa, 1)$. We need to show that $\lambda \in \operatorname{Hom}_{N_{\alpha} \cap R}(\kappa, 1)$. According to Lemma 12.8, it suffices to show that $\lambda$ is invariant under $N_{\alpha} \cap B_{0, r-1}$ and $N_{\alpha} \cap J_{i}$, for $1 \leqslant i \leqslant r-1$. Fix $x \in N_{\alpha} \cap B_{0, r-1}$. Proposition 2 of [9] implies that:

$$
N_{\alpha} \cap B_{0, r-1} \subset\left(H_{\alpha} \cap B_{0, r-1}\right) B_{1, r-1} .
$$

Thus we may choose $x^{\prime} \in H_{\alpha} \cap B_{0, r-1}$ so that $x \in x^{\prime} B_{1, r-1}$. Since $\kappa_{r} \mid B_{1, r-1} \equiv 1$, we have $\kappa_{r}(x)=\kappa_{r}\left(x^{\prime}\right)$. On the other hand, if $j \neq r$ then $\kappa_{j}(x)=\hat{\rho}_{j}\left(\xi_{j}(x)\right)=$ $\hat{\rho}_{j}\left(\xi_{j}\left(x^{\prime}\right)\right)=\kappa_{j}\left(x^{\prime}\right)$, where $\hat{\rho}_{j}$ is the Weil representation defined in Section 8. Therefore, $\kappa(x)=\kappa\left(x^{\prime}\right)$, from which it follows that if $\lambda$ is invariant under $H_{\alpha} \cap B_{0, r-1}$ it must also be invariant under $N_{\alpha} \cap B_{0, r-1}$. Now suppose $1 \leqslant i \leqslant r-1$ and fix $x \in H_{\alpha} \cap J_{i}$. According to Lemma 12.7, we may choose $x^{\prime} \in H_{\alpha} \cap J_{i}$ such that $x \in x^{\prime} J_{i}^{\prime \prime}$. We have $\kappa_{i}(x)=\kappa_{i}\left(x^{\prime}\right)$ and, furthermore, Lemma 12.9 implies $\kappa_{j}(x)=$ $\kappa_{j}\left(x^{\prime}\right)=1$, when $j \neq i$. Again, we have $\kappa(x)=\kappa\left(x^{\prime}\right)$. Thus $\lambda$ must be invariant under $N_{\alpha} \cap J_{i}$.

LEMMA 12.11. If $\ell(\alpha)>1$ then $\operatorname{Hom}_{H_{\alpha} \cap R}(\kappa, 1)=0$.

Proof. Suppose $\operatorname{Hom}_{H_{\alpha} \cap R}(\kappa, 1) \neq 0$. According to Lemma 12.10, this implies that $\operatorname{Hom}_{N_{\alpha} \cap R}(\kappa, 1) \neq 0$. Choose a nonzero linear form $\lambda \in \operatorname{Hom}_{N_{\alpha} \cap R}(\kappa, 1)$. Define a linear form on the space of $\pi=\operatorname{Ind}_{R}^{G}(\kappa)$ by 


$$
\Lambda(f)=\sum_{x \in\left(N_{\alpha} \cap R\right) \backslash N_{\alpha}} \lambda(f(x)) .
$$

Then since $\Lambda \in \operatorname{Hom}_{N_{\alpha}}(\pi, 1)$ it must factor through the Jacquet module of $\pi$ associated to $N_{\alpha}$. Supercuspidality implies that the Jacquet module is zero and thus $\Lambda=0$. On the other hand, we can show also that $\Lambda$ is nonzero as follows. Choose $v \in V$ such that $\lambda(v) \neq 0$. Define $f$ by $f(g)=\kappa(g) v$, when $g \in R$, and $f(g)=0$ when $g \in G-R$. Then $\Lambda(f)=\lambda(v) \neq 0$. Our assertion follows from this contradiction.

The following is a modification of Proposition 10.1:

LEMMA 12.12. Assume $f_{r}=1, E / E^{\prime}$ is ramified and $n_{r-1}$ is even. Suppose $\alpha \in \mathcal{A}_{\eta}$ is such that

$$
\varpi^{\alpha}=\varpi_{r-1}^{a}\left(\left(\begin{array}{cc}
0 & 1 \\
-1 & 0
\end{array}\right) \oplus \cdots \oplus\left(\begin{array}{cc}
0 & 1 \\
-1 & 0
\end{array}\right)\right) \in G_{r-1},
$$

(with $n_{r-1} / 2$ summands) for some odd integer a. If $i \in\{1, \ldots, r-1\}$ and $\kappa_{i}=\kappa_{i}^{v}$ for some special isomorphism $v$ then $\operatorname{Hom}_{J_{i} \cap H_{\alpha}}\left(\kappa_{i}, 1\right)=\operatorname{Hom}_{J_{i} \cap H_{\alpha}}\left(\rho_{i}, 1\right)$ has dimension one.

Proof. Under the stated hypotheses, let $W_{i \alpha}^{+}$and $W_{i \alpha}^{-}$be defined by analogy with $W_{i}^{+}$and $W_{i}^{-}$, replacing $\mathfrak{h}$ by $\mathfrak{h}_{\alpha}=g_{\alpha} \mathfrak{h} g_{\alpha}^{-1}$. It follows from Lemma $8.1(\mathrm{v})$ that $c_{i} \in E^{\prime}=E_{r-1} \cap \mathfrak{h}_{\alpha}$. Using this fact and imitating the proof of Lemma 8.2, we deduce that $W_{i \alpha}^{+}$and $W_{i \alpha}^{-}$form a polarization of $W_{i}$. By definition, $\operatorname{Hom}_{J_{i} \cap H_{\alpha}}\left(\kappa_{i}, 1\right)=\operatorname{Hom}_{J_{i} \cap H_{\alpha}}\left(\rho_{i}, 1\right)$. Using a Cayley transform, as in Lemma 10.3, we see that $\operatorname{Hom}_{J_{i} \cap H_{\alpha}}\left(\rho_{i}, 1\right)=\operatorname{Hom}_{W_{i \alpha}^{-}}\left(\rho_{i}, 1\right)$. Our claim now is a consequence of Proposition 7.2.

LEMMA 12.13. Under the hypotheses of Lemma 12.12, $\operatorname{Hom}_{R \cap H_{\alpha}}(\kappa, 1)=0$, regardless of which special isomorphisms are chosen to define $\kappa_{1}, \ldots, \kappa_{r-1}$.

Proof. According to Lemma 12.12, we may fix for each $i \in\{1, \ldots, r-1\}$ a nonzero linear form $\mu_{i} \in \operatorname{Hom}_{J_{i} \cap H_{\alpha}}\left(\kappa_{i}, 1\right)$ and, up to scalar multiples, these choices are unique. Now define a representation $\rho_{(r)}$ of the group $J_{(r)}=J_{1} \cdots J_{r-1}$ on the space $V_{(r)}=V_{1} \otimes \cdots \otimes V_{r-1}$ by restricting $\kappa_{1} \otimes \cdots \otimes \kappa_{r-1}$ to $J_{(r)}$. We claim that every $\left(J_{(r)} \cap H_{\alpha}\right)$-invariant linear form on $V_{(r)}$ is a multiple of the linear form given on elementary tensors by

$$
\mu_{(r)}\left(v_{1} \otimes \cdots \otimes v_{r-1}\right)=\mu_{1}\left(v_{1}\right) \cdots \mu_{r-1}\left(v_{r-1}\right) .
$$

For $r=2$, this is obvious. For $r>2$, we use induction on $r$ and argue as follows. Let $\mu^{\prime}$ be a nonzero $\left(J_{(r)} \cap H_{\alpha}\right)$-invariant linear form on $V_{(r)}$. For each $v_{1} \in V_{1}$, define a linear form $\mu_{v_{1}}^{\prime}$ on $V_{(r)}^{(1)}=V_{2} \otimes \cdots \otimes V_{r-1}$ by

$$
\mu_{v_{1}}^{\prime}\left(v_{2} \otimes \cdots \otimes v_{r-1}\right)=\mu^{\prime}\left(v_{1} \otimes \cdots \otimes v_{r-1}\right) .
$$

Let $J_{(r)}^{(1)}=J_{2} \cdots J_{r-1}$ and define a representation $\rho_{(r)}^{(1)}$ of $J_{(r)}^{(1)}$ on $V_{(r)}^{(1)}$ by restricting $\kappa_{2} \otimes \cdots \otimes \kappa_{r-1}$ to $J_{(r)}^{(1)}$. Note that $v_{1}$ is $\left(J_{(r)}^{(1)} \cap H_{\alpha}\right)$-fixed, since $J_{(r)}^{(1)} \cap H_{\alpha} \subset B_{1}$ and $\theta_{1} \circ \operatorname{det}_{1}$ is trivial on $J_{(r)}^{(1)} \cap H_{\alpha}$. It follows that $\mu_{v_{1}}^{\prime} \in \operatorname{Hom}_{J_{(r)}^{(1)} \cap H_{\alpha}}\left(\rho_{(r)}, 1\right)$. By induction, there must exist a constant $c\left(v_{1}\right)$ such that 


$$
\mu_{v_{1}}^{\prime}\left(v_{2} \otimes \cdots \otimes v_{r-1}\right)=c\left(v_{1}\right) \mu_{2}\left(v_{2}\right) \cdots \mu_{r-1}\left(v_{r-1}\right) .
$$

In fact, as $v_{1}$ varies $c\left(v_{1}\right)$ defines a linear form on $V_{1}$.

Note that $\rho_{(r)}^{(1)}$ is a multiple of the character $1+x \mapsto\left\langle c_{(r)}^{(1)}, x\right\rangle$ on $J_{1}$, where $c_{(r)}^{(1)}=c_{2}+\cdots+c_{r-1}$. Lemma 8.1(v) implies $c_{(r)}^{(1)} \in E_{r-1}^{\prime}=E_{r-1} \cap \mathfrak{h}_{\alpha}$. Using a Cayley transform, as in Lemma 10.3, one deduces that $\rho_{(r)}^{(1)} \mid J_{1} \cap H_{\alpha} \equiv 1$. Therefore, if $x \in J_{1} \cap H_{\alpha}$, then

$$
\begin{aligned}
c\left(\rho_{1}(x) v_{1}\right) \mu_{2}\left(v_{2}\right) \cdots \mu_{r-1}\left(v_{r-1}\right) & =\mu^{\prime}\left(\left(\rho_{1}(x) v_{1}\right) \otimes v_{2} \otimes \cdots \otimes v_{r-1}\right) \\
& =\mu^{\prime}\left(\left(\kappa_{1}(x) v_{1}\right) \otimes \cdots \otimes\left(\kappa_{r-1}(x) v_{r-1}\right)\right) \\
& =\mu^{\prime}\left(v_{1} \otimes \cdots \otimes v_{r-1}\right) \\
& =c\left(v_{1}\right) \mu_{2}\left(v_{2}\right) \cdots \mu_{r-1}\left(v_{r-1}\right) .
\end{aligned}
$$

It follows that $c \in \operatorname{Hom}_{J_{1} \cap H_{\alpha}}\left(\rho_{1}, 1\right)$ and thus is a multiple of $\mu_{1}$. We have now shown, as we claimed above, that every $\left(J_{(r)} \cap H_{\alpha}\right)$-invariant linear form on $V_{(r)}$ is a multiple of $\mu_{(r)}$.

Now suppose $\mu \in \operatorname{Hom}_{R \cap H_{\alpha}}(\kappa, 1)$ is nonzero. For each $v_{r} \in V_{r}$, let $\mu_{v_{r}}$ be the linear form on $V_{(r)}$ given by $\mu_{v_{r}}\left(v_{1} \otimes \cdots \otimes v_{r-1}\right)=\mu\left(v_{1} \otimes \cdots \otimes v_{r}\right)$. Since $\left(J_{(r)} \cap H_{\alpha}\right) \subset B_{1}$, the element $v_{r}$ is fixed by $J_{(r)} \cap H_{\alpha}$. Therefore, $\mu_{v_{r}}$ is $\left(J_{(r)} \cap H_{\alpha}\right)$-invariant and therefore is a multiple of $\mu_{(r)}$. Say $\mu_{v_{r}}=\mu_{r}\left(v_{r}\right) \mu_{(r)}$, for some constant $\mu_{r}\left(v_{r}\right)$. Then $\mu_{r}$ is a linear form on $V_{r}$. Since $\mu$ is nonzero, we may choose $v_{1} \in V_{1}, \ldots, v_{r} \in V_{r}$ such that $\mu_{i}\left(v_{i}\right) \neq 0$, for all indices $i$. Suppose $x \in B_{0, r-1} \cap H_{\alpha}$. We have

$$
\begin{aligned}
\mu_{1}\left(v_{1}\right) \cdots \mu_{r}\left(v_{r}\right) & =\mu\left(v_{1} \otimes \cdots \otimes v_{r}\right) \\
& =\mu\left(\left(\kappa_{1}(x) v_{1}\right) \otimes \cdots \otimes\left(\kappa_{r}(x) v_{r}\right)\right) \\
& =\mu_{1}\left(\kappa_{1}(x) v_{1}\right) \cdots \mu_{r}\left(\kappa_{r}(x) v_{r}\right) .
\end{aligned}
$$

Note that we must have $\mu_{i}\left(\kappa_{i}(x) v_{i}\right) \neq 0$, for all $i$ and thus we may define

$$
\chi(x)=\frac{\mu_{1}\left(v_{1}\right) \cdots \mu_{r-1}\left(v_{r-1}\right)}{\mu_{1}\left(\kappa_{1}(x) v_{1}\right) \cdots \mu_{r-1}\left(\kappa_{r-1}(x) v_{r-1}\right)}=\frac{\mu_{r}\left(\kappa_{r}(x) v_{r}\right)}{\mu_{r}\left(v_{r}\right)} .
$$

As the notation suggests, $\chi(x)$ depends on $x$, but does not depend on $v_{1}, \ldots, v_{r}$. The relation $\mu_{r}\left(\kappa_{r}(x) v_{r}\right)=\chi(x) \mu_{r}\left(v_{r}\right)$ implies that $\chi$ is a character of $B_{0, r-1} \cap H_{\alpha}$. In fact, since $\kappa_{r} \mid B_{1, r-1} \cap H_{\alpha}$ is a multiple of the trivial representation, $\chi$ may be viewed as a character of the finite symplectic group $\mathcal{B}_{\alpha}=\left(B_{0, r-1} \cap H_{\alpha}\right) /\left(B_{1, r-1} \cap H_{\alpha}\right)$. Therefore, $\chi$ is trivial and so $\mu_{r}$ must be a nonzero element of $\operatorname{Hom}_{B_{0, r-1} \cap H_{\alpha}}\left(\kappa_{r}, 1\right)$. The latter space is identical to $\operatorname{Hom}_{\mathcal{B}_{\alpha}}\left(\rho_{r}, 1\right)$, where $\rho_{r}$ is the cuspidal representation of $\mathcal{G}=B_{0, r-1} / B_{1, r-1} \cong \mathrm{GL}\left(n_{r-1}, k_{r-1}\right)$ associated to $\kappa_{r}$. Since $\rho_{r}$ is cuspidal, it must have a Gelfand-Graev model, that is, an embedding in $\operatorname{Ind}_{\mathcal{N}}^{\mathcal{G}}(\psi)$, where $\mathcal{N}$ is the unipotent radical of a Borel subgroup of $\mathcal{G}$ and $\psi$ is a nondegenerate character. Theorem A of [21] now implies that $\rho_{r}$ cannot have an embedding in $\operatorname{Ind}_{\mathcal{B}_{\alpha}}^{\mathcal{G}}(1)$. But Frobenius reciprocity implies

$$
\operatorname{Hom}_{\mathcal{G}}\left(\rho_{r}, \operatorname{Ind}_{\mathcal{B}_{\alpha}}^{\mathcal{G}}(1)\right) \cong \operatorname{Hom}_{\mathcal{B}_{\alpha}}\left(\rho_{r}, 1\right) .
$$


Therefore, we deduce that $\operatorname{Hom}_{\mathcal{B}_{\alpha}}\left(\rho_{r}, 1\right)=0$, which contradicts the fact that $\mu_{r} \in \operatorname{Hom}_{\mathcal{B}_{\alpha}}\left(\rho_{r}, 1\right)$ is nonzero.

Proof of Proposition 12.1. As observed in the remarks at the beginning of this section, we are reduced to showing that if $g \in G_{r-1}$ and $\operatorname{Hom}_{R \cap g g^{-1}}(\kappa, 1) \neq 0$ then $g$ must lie in $R H$. According to Lemma 12.4, we may assume $g=g_{\alpha}$ for some $\alpha \in \mathcal{A}_{\eta}$. But Lemma 12.6 says that if $\alpha \in \mathcal{A}_{\eta}$ and $g_{\alpha} \notin R_{r-1} H_{r-1}$ then either $\ell(\alpha)>1$ or $\ell(\alpha)=1, E / E^{\prime}$ is ramified, $n_{r-1}$ is even and $\left.\varpi^{\alpha}=\varpi_{r-1}^{a}\left(\begin{array}{cc}0 & 1 \\ -1 & 0\end{array}\right) \oplus \cdots \oplus\left(\begin{array}{cc}0 & 1 \\ -1 & 0\end{array}\right)\right)$ for some odd integer $a$. In the former case, we apply Lemma 12.11 and in the latter case we apply Lemma 12.13 .

\section{Factorization of Linear Forms}

At this point, the proof of Theorem 3.3 has been reduced to the task of proving that $\operatorname{Hom}_{R \cap H}(\kappa, 1)$ has dimension one. In view of Propositions 10.1 and 12.2, we may assume $r>1$. Our argument involves an induction on the length of the Howe factorization of $\theta$. In particular, we use the fact that $\theta^{(1)}=\theta_{1}^{E} \cdots \theta_{r-1}^{E}$ is a quasicharacter of $E^{\times}$which is admissible over $E_{1}$ and has a shorter Howe factorization than that of $\theta$. Most of the main ideas used in this section are already evident in the proof of Lemma 12.13. It should be understood in this section that we have fixed, once and for all, our choices of special isomorphisms. None of the arguments will depend on these choices. Note that the choice of special isomorphism used to define a given $\kappa_{i}$ does not affect the definition of the representation space $V_{i}$, viewed as a set and not as an $R$-module. However, it may affect the character $\vartheta_{i}$ occurring in Proposition 10.1. Nonetheless, it turns out that for all $\alpha \in \mathcal{A}_{\eta}$ the space $\operatorname{Hom}_{R \cap H_{\alpha}}(\kappa, 1)$ does not depend on the choices of special isomorphisms. When $g_{\alpha} \notin R H$, this results from Lemma 12.11 and Lemma 12.13 which establish that $\operatorname{Hom}_{R \cap H_{\alpha}}(\kappa, 1)$ vanishes. When $g_{\alpha} \in R H$, we may as well assume $g_{\alpha}=1$ and thus $H_{\alpha}=H$. The main result of this section, Proposition 13.1, gives a description of $\operatorname{Hom}_{R \cap H}(\kappa, 1)$ which is independent of the choices of special isomorphisms.

Let us make the following definitions:

$$
\kappa^{(1)}=\kappa_{2} \otimes \cdots \otimes \kappa_{r}, \quad R^{(1)}=E^{\times} B\left(\theta^{(1)}\right), \quad V^{(1)}=V_{2} \otimes \cdots \otimes V_{r} .
$$

For each $i$ with $f_{i}>1$, we have defined a linear form $\lambda_{i}$ on $V_{i}$ by $\lambda_{i}(\varphi)=\varphi(1)$ and we have shown that $\lambda_{i}$ generates the one-dimensional spaces $\operatorname{Hom}_{R \cap H}\left(\kappa_{i}, \vartheta_{i}\right)=$ $\operatorname{Hom}_{B\left(\theta_{i}\right) \cap H}\left(\kappa_{i}, 1\right)$. (See Proposition 7.2, Section 8 and Proposition 10.1.) When $f_{i}$ is even then $V_{i}=\mathrm{C}$ and $\lambda_{i}$ is just the identity map on $\mathrm{C}$. When $f_{i}=1$, and thus $i=r$, fix a nonzero element

$$
\lambda_{r} \in \operatorname{Hom}_{R \cap H}\left(\kappa_{r}, \vartheta_{r}\right)=\operatorname{Hom}_{B_{0, r-1} \cap H}\left(\kappa_{r}, 1\right),
$$

where $\vartheta_{r}=\prod_{i=1}^{r-1} \vartheta_{i}$, where $\vartheta_{i}$ is chosen as in Proposition 10.1. According to Proposition 12.3, such a linear form $\lambda_{r}$ exists and is unique up to scalar multiples. Note that, for general $i \in\{1 \ldots, r\}$, if $\vartheta_{i}$ is nontrivial then the identity $\operatorname{Hom}_{R \cap H}\left(\kappa_{i}, \vartheta_{i}\right)=$ 
$\operatorname{Hom}_{B\left(\theta_{i}\right) \cap H}\left(\kappa_{i}, 1\right)$ implies that $\operatorname{Hom}_{R \cap H}\left(\kappa_{i}, 1\right)=0$, as $\operatorname{Hom}_{R \cap H}\left(\kappa_{i}, 1\right) \subset$ $\operatorname{Hom}_{B\left(\theta_{i}\right) \cap H}\left(\kappa_{i}, 1\right)$ and $\operatorname{Hom}_{B\left(\theta_{i}\right) \cap H}\left(\kappa_{i}, 1\right)$ has dimension one.

PROPOSITION 13.1. The spaces $\operatorname{Hom}_{R \cap H}(\kappa, 1)$ and $\operatorname{Hom}_{B(\theta) \cap H}(\kappa, 1)$ are identical and have dimension 1. Given $\lambda \in \operatorname{Hom}_{B(\theta) \cap H}(\kappa, 1)$, there exists a constant $c$ such that

$$
\lambda\left(v_{1} \otimes \cdots \otimes v_{r}\right)=c \lambda_{1}\left(v_{1}\right) \cdots \lambda_{r}\left(v_{r}\right),
$$

for all $v_{1} \in V_{1}, \ldots, v_{r} \in V_{r}$.

Proof. Fix $\lambda \in \operatorname{Hom}_{B(\theta) \cap H}(\kappa, 1)$. It suffices to show that $\lambda$ has the desired factorization, since it will then follow that $\lambda$ is $(R \cap H)$-invariant. (Note that $\lambda_{i} \in \operatorname{Hom}_{R \cap H}\left(\kappa_{i}, \vartheta_{i}\right)$ implies that $\lambda \in \operatorname{Hom}_{R \cap H}(\kappa, 1)$, since $\prod_{i=1}^{r} \vartheta_{i}=1$.) We will prove this by induction on $r$. The case of $r=1$ is established in Proposition 10.1 and Proposition 12.2. Therefore, we assume $r>1$. Assume, in addition, that $\lambda$ is nonzero.

Let us consider first the case in which $f_{r}>1$. Note that the characters $\vartheta_{i}$ in Proposition 10.11 are all trivial in this case. Given $v_{1} \in V_{1}$, define a linear form $\lambda_{v_{1}}^{(1)}$ on $V^{(1)}$ by the condition $\lambda_{v_{1}}^{(1)}\left(v_{2} \otimes \cdots \otimes v_{r}\right)=\lambda\left(v_{1} \otimes \cdots \otimes v_{r}\right)$ on elementary tensors. Since $B\left(\theta^{(1)}\right) \subset B_{1}$ and $\theta_{1} \circ$ det $_{1}$ is trivial on $B_{1} \cap H$, it must be that $v_{1}$ is $\left(B\left(\theta^{(1)}\right) \cap H\right)$-fixed. Consequently, the linear form $\lambda_{v_{1}}^{(1)}$ must lie in $\operatorname{Hom}_{B\left(\theta^{(1)} \cap H\right.}\left(\kappa^{(1)}, 1\right)$. From the inductive assumption, there exists a constant $c\left(v_{1}\right)$ such that

$$
\lambda_{v_{1}}^{(1)}\left(v_{2} \otimes \cdots \otimes v_{r}\right)=c\left(v_{1}\right) \lambda_{2}\left(v_{2}\right) \cdots \lambda_{r}\left(v_{r}\right)
$$

Clearly, $c$ is a linear form on $V_{1}$. If $x \in B_{\ell_{1}} \cap H$, then $\kappa^{(1)}(x)$ acts trivially on $V^{(1)}$, since $c^{(1)}=c_{2}+\cdots+c_{r} \in \mathfrak{h}^{\perp}$ and $\kappa^{(1)}$ is a multiple of the character $1+x \mapsto\left\langle c_{i}, x\right\rangle$ on $B_{\ell_{1}}$. Hence,

$$
\begin{aligned}
c\left(\kappa_{1}(x) v_{1}\right) \lambda_{2}\left(v_{2}\right) \cdots \lambda_{r}\left(v_{r}\right) & =\lambda\left(\left(\kappa_{1}(x) v_{1}\right) \otimes v_{2} \otimes \cdots \otimes v_{r}\right) \\
& =\lambda\left(\left(\kappa_{1}(x) v_{1}\right) \otimes \cdots \otimes\left(\kappa_{r}(x) v_{r}\right)\right) \\
& =\lambda\left(v_{1} \otimes \cdots \otimes v_{r}\right) \\
& =c\left(v_{1}\right) \lambda_{2}\left(v_{2}\right) \cdots \lambda_{r}\left(v_{r}\right) .
\end{aligned}
$$

Therefore, $c \in \operatorname{Hom}_{B_{\ell_{1}} \cap H}\left(\kappa_{1}, 1\right)$ and thus $c$ is a multiple of $\lambda_{1}$. Our claim now follows in the case $f_{r}>1$.

Now assume $f_{r}=1$. Let $\kappa_{(r)}=\kappa_{1} \otimes \cdots \otimes \kappa_{r-1}$ be the representation of $B_{(r)}=B\left(\theta_{1}\right) \cdots B\left(\theta_{r-1}\right)$ on the space $V_{(r)}=V_{1} \otimes \cdots \otimes V_{r-1}$. According to an argument similar to the one just given above, every $\left(B_{(r)} \cap H\right)$-invariant linear form on $V_{(r)}$ must be a multiple of the linear form $\lambda_{(r)}$ defined by the condition

$$
\lambda_{(r)}\left(v_{1} \otimes \cdots \otimes v_{r-1}\right)=\lambda_{1}\left(v_{1}\right) \cdots \lambda_{r-1}\left(v_{r-1}\right) .
$$

Suppose $v_{r} \in V_{r}$. Since $v_{r}$ is $\left(R \cap B_{1}\right)$-fixed and $B_{(r)} \subset R \cap B_{1}$, it follows that $v_{r}$ must be $B_{(r)}$-fixed. We therefore obtain a $\left(B_{(r)} \cap H\right)$-invariant linear form $\lambda_{v_{r}}$ on $V_{(r)}$ by the condition: $\lambda_{v_{r}}\left(v_{1} \otimes \cdots \otimes v_{r-1}\right)=\lambda\left(v_{1} \otimes \cdots \otimes v_{r}\right)$. There must be a constant $c\left(v_{r}\right)$ such that $\lambda_{v_{r}}=c\left(v_{r}\right) \lambda_{(r)}$. In fact, $c$ is a linear form on $V_{r}$. Now fix $v_{1}, \ldots, v_{r-1}$ so that $\lambda_{1}\left(v_{1}\right), \ldots, \lambda_{r-1}\left(v_{r-1}\right)$ are nonzero. Then since $\lambda \in \operatorname{Hom}_{B(\theta) \cap H}(\kappa, 1)$ and $\lambda_{i} \in \operatorname{Hom}_{R \cap H}\left(\kappa_{i}, \vartheta_{i}\right)$, when $1 \leqslant i \leqslant r-1$, and 


$$
c\left(v_{r}\right)=\frac{\lambda\left(v_{1} \otimes \cdots \otimes v_{r}\right)}{\lambda_{1}\left(v_{1}\right) \cdots \lambda_{r-1}\left(v_{r-1}\right)},
$$

we deduce that $c \in \operatorname{Hom}_{B_{0, r-1} \cap H}\left(\kappa_{r}, \vartheta_{r}\right)$. Therefore, according to Proposition 12.3, $c$ is a multiple of $\lambda_{r}$ and our claim follows.

\section{Final Remarks on the Proofs of Theorems 3.3 and 4.4}

Suppose $\pi$ is an irreducible, tame supercuspidal representation of $G$ and suppose $H$ is a unitary group in $G$. To prove Theorem 4.4 , according to the discussion after its statement, it suffices to show that if $\pi$ is Galois invariant then $\pi$ is $H$-distinguished. The representation $\pi$ is induced from some representation $\kappa=\kappa_{0} \otimes \cdots \otimes \kappa_{r}$ of a subgroup $R$, where $\kappa$ and $R$ are defined in Section 8. From Lemma 11.1, we see that we may assume $\kappa_{0}=1$. Then, according to Lemma 11.2 and Proposition 12.1, we have $\operatorname{Hom}_{H}(\pi, 1) \cong \operatorname{Hom}_{R \cap H}(\kappa, 1)$. Next, Proposition 13.1 shows that $\operatorname{Hom}_{R \cap H}(\kappa, 1)$ has dimension one. Thus, we have shown that if $\pi$ is Galois invariant then $\operatorname{Hom}_{H}(\pi, 1)$ has dimension one, which completes the proof of Theorem 4.4. Moreover, we have shown that if $\operatorname{Hom}_{H}(\pi, 1)$ is nonzero then it has dimension one. Therefore, we have also demonstrated Theorem 3.3.

\section{Existence of Distinguished Representations}

As usual, we assume $E / F$ is a tamely ramified extension of $p$-adic fields with $p \neq 2$. The purpose of this section is to prove:

PROPOSITION 15.1 There exists a quasicharacter $\theta$ of $E^{\times}$which is admissible over $F$ and is trivial on $U\left(1, E / E^{\prime}\right)$.

This result will be an immediate consequence of two lemmas proved below and our approach is a rather straightforward generalization of the techniques used by Jeff Adler in another setting (see [1]). By the results presented in previous sections of this paper, the characters in Proposition 15.1 correspond to supercuspidal representations $\pi$ such that $\pi \sim \pi \circ \imath$ and they are therefore distinguished.

Let $L_{1}, \ldots, L_{r}$ be the maximal proper intermediate fields of $E / F$ (thus $F \subseteq L_{i} \subsetneq E$ ). Define closed subgroups $C_{1}, \ldots, C_{r}$ of $E^{\times}$by taking $C_{i}$ to be the kernel of the norm map from $E^{\times}$to $L_{i}^{\times}$, unless $E / L_{i}$ is ramified in which case $C_{i}$ is the kernel of the norm map from $1+\mathfrak{F}_{E}$ to $1+\mathfrak{S}_{L_{i}}$. Then a quasicharacter $\theta$ of $E^{\times}$is admissible precisely when it is nontrivial on all of these subgroups.

Now suppose we are given $t$ as in the statement of Lemma 4.3 above. Assume we are also given an automorphism $\sigma$ of $E$ of order two which agrees with $\imath$ on $F$. Let $E^{\prime}$ be the fixed field of $\sigma$. (Then $E^{\prime}$ cannot contain $F$.)

LEMMA 15.2 ([1], Lemma 6.2). Let A be a topological Abelian group with closed subgroups $C_{1}, \ldots, C_{r}$ and $N$, such that $N$ does not contain any $C_{i}$. Then there exists a character $\theta$ of $A$ which is trivial on $N$ and nontrivial on every $C_{i}$. 
We will use this when $A=E^{\times}, C_{i}$ is defined as above and $N=U\left(1, E / E^{\prime}\right)$. This requires the following lemma.

LEMMA 15.3. $U\left(1, E / E^{\prime}\right)$ does not contain any of the subgroups $C_{i}$.

Proof. Suppose that $U\left(1, E / E^{\prime}\right) \supset C_{i}$, for some $i$. Let $L_{0}=L_{i} \cap E^{\prime}$. We define integers $a, b, c \geqslant 2$ by

$$
a=\left[E: L_{i}\right], \quad b=\left[E^{\prime}: L_{0}\right] \text { and } c=\left[L_{i}: L_{0}\right] .
$$

Then $2 b=a c$. As manifolds over $L_{0}$, the dimensions of $\mathrm{U}\left(1, E / E^{\prime}\right)$ and $C_{i}$ are $b$ and $(a-1) c$. Thus we have $a / 2=b / c \geqslant a-1$. It follows that $a=2$ and $b=c$. Choose $u \in \mathfrak{P}_{E^{\prime}}-\mathfrak{P}_{L_{0}}$ and let $x=(1+u) /(1+\tilde{u}) \in C_{i}$, where $\tilde{u}$ is the image of $u$ under the nontrivial Galois automorphism of $E / L_{i}$. Since $C_{i} \subset \mathrm{U}\left(1, E / E^{\prime}\right)$, we must have $1=N_{E / E^{\prime}}(x)=x^{2}$, and thus $x= \pm 1$. If $x=1$ then $u=\tilde{u} \in L_{i} \cap E^{\prime}=L_{0}$, which is false. On the other hand, if $x=-1$ then it follows from the identity $1+u=$ $-1-\tilde{u}$ that $|2|<1$. Hence, our claim follows.

\section{References}

1. Adler, J.: Self-contragredient supercuspidal representations of $\mathrm{GL}_{n}$, Proc. Amer. Math. Soc. 125 (1997), 2471-2479.

2. Arthur, J. and Clozel, L.: Simple Algebras, Base Change, and the Advanced Theory of the Trace Formula, Ann. of Math. Stud. 120, Princeton Univ. Press, Princeton, N.J., 1989.

3. Deligne, P. and Lusztig, G.: Representations of reductive groups over a finite field, Ann. Math. 103 (1976), 103-161.

4. Doi, K. and Naganuma, H.: On the functional equation of certain Dirichlet series, Invent. Math. 9 (1969), 1-14.

5. Flicker, Y.: On distinguished representations, J. Reine Angew. Math. 418 (1991), 139-172.

6. Gérardin, P.: Weil representations associated to finite fields, J. Algebra 46 (1977), 54-101.

7. Gow, R.: Two multiplicity-free permutation presentations of the general linear group GL $\left(n, q^{2}\right)$, Math. Z. 188(1) (1984), 45-54.

8. Hakim, J. and Mao, Z.: Cuspidal representations associated to $(\mathrm{GL}(n), \mathrm{O}(n))$ over finite fields and p-adic fields, J. Algebra 213 (1999), 129-143.

9. Hakim, J. and Mao, Z.: Supercuspidal representations of GL $(n)$ distinguished by a unitary subgroup, Pacific J. Math. 185 (1998), 149-162.

10. Hakim, J. and Murnaghan, F.: Globalization of distinguished supercuspidal representations of GL(n), Canad. Math. Bull., to appear.

11. Hakim, J. and Murnaghan, F.: Tame supercuspidal representations of GL( $n)$ distinguished by an orthogonal group, work in progress.

12. Hakim, J. and Murnaghan, F.: Two types of distinguished tame supercuspidal representations of GL(n), Internat. Math. Res. Notices to appear.

13. Harder, G., Langlands, R. and Rapoport, M.: Algebraische Zyklen auf Hilbert-Blumenthal-Flächen, J. Reine Angew. Math. 366 (1986), 53-120.

14. Heumos, M. and Rallis, S.: Symplectic-Whittaker models for $\mathrm{GL}_{n}$, Pacific J. Math. 146(2) (1990), 247-279.

15. Howe, R.: Tamely ramified supercuspidal representations of $\mathrm{GL}_{n}$, Pacific J. Math. 73(2) (1977), 437-460.

16. Jacobowitz, R.: Hermitian forms over local fields, Amer. J. Math. 84 (1962), 441-465. 
17. Jacquet, H.: Automorphic Forms on GL(2), Part II, Lecture Notes in Math. 278, Springer, New York, 1978.

18. Jacquet, H. and Rallis, S.: Symplectic periods, J. Reine Angew. Math. 423 (1992), 175-197.

19. Jacquet, H. and Ye, Y.: Distinguished representations and quadratic base change for GL(3), Trans. Amer. Math. Soc. 348(3) (1996), 913-939.

20. Jacquet, H. and Ye, Y.: Une remarque sur le changement de base quadratique, $C . R$. Acad. Sci. Paris Série I 311 (1990), 671-676.

21. Klyachko, A. A.: Models for the complex representations of the groups $\operatorname{GL}(n, q)$, Math. USSR Sb. 48(2) (1984), 365-379.

22. Langlands, R.: Base Change for GL(2), Ann. of Math. Stud. 96, Princeton Univ. Press, Princeton, N.J., 1980

23. Lusztig, G.: Symmetric spaces over a finite field, In: P. Cartier et al. (eds), The Grothendieck Festschrift Vol. III, Birkhäuser, Basel, 1990, pp. 57-81.

24. Moy, A.: Local constants and the tame Langlands correspondence, Amer. J. Math. 64 (1991), 863-930.

25. Murnaghan, F. and Repka, J.: Reducibility of some induced representations of $p$-adic unitary groups, Trans. Amer. Math. Soc. 351 (1999), 193-210.

26. Murnaghan, F. and Repka, J.: Reducibility of some induced representations of split classical groups, Compositio Math. 114 (1998), 263-313.

27. Prasad, D.: On a conjecture of Jacquet about distinguished representations of GL $(n)$, Preprint.

28. Saito, H.: Automorphic forms and algebraic extensions of number fields, Lectures in Math., Kyoto University, 1975.

29. Serre, J.-P.: Local Fields, Grad. Texts in Math. 67, Springer-Verlag, New York, 1979.

30. Shintani, T.: On liftings of holomorphic cusp forms, Proc. Sympos. Pure Math. 33(2) (1979), 79-110.

31. Yu, J.-K.: Construction of tame supercuspidal representations, J. Amer. Math. Soc. 14(3) (2001), 579-622. 